ARGONNE NATIONAL LABORATORY

9700 South Cass Avenue

Argonne, Illinois 60440

ELMOE: AN IBM-704 PROGRAM

TREATING ELASTIC SCATTERING RESONANCES

IN FAST REACTORS

by

A. L. Rago

Applied Mathematics Division

and

H. H. Hummel

Reactor Physics Division

January 1964

Operated by The University of Chicago

under

Contract W-31-109-eng- 38

with the

U. S. Atomic Energy Commission 


\section{DISCLAIMER}

This report was prepared as an account of work sponsored by an agency of the United States Government. Neither the United States Government nor any agency Thereof, nor any of their employees, makes any warranty, express or implied, or assumes any legal liability or responsibility for the accuracy, completeness, or usefulness of any information, apparatus, product, or process disclosed, or represents that its use would not infringe privately owned rights. Reference herein to any specific commercial product, process, or service by trade name, trademark, manufacturer, or otherwise does not necessarily constitute or imply its endorsement, recommendation, or favoring by the United States Government or any agency thereof. The views and opinions of authors expressed herein do not necessarily state or reflect those of the United States Government or any agency thereof. 


\section{DISCLAIMER}

Portions of this document may be illegible in electronic image products. Images are produced from the best available original document. 
TABLE OF CONTENTS

$\underline{\text { Page }}$

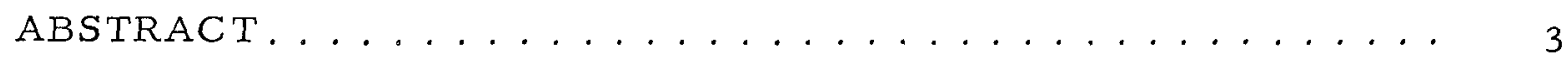

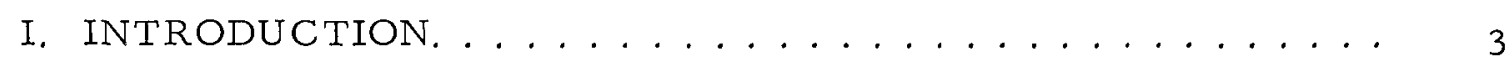

II. CALCULATION OF CROSS SECTIONS .......... 5

III. FLUX APPROXIMATIONS . . . . . . . . . . . . . 10

IV. TREATMENT OF NARROW RESONANCES .......... 15

V. LIBRARY TAPE. ....................... 16

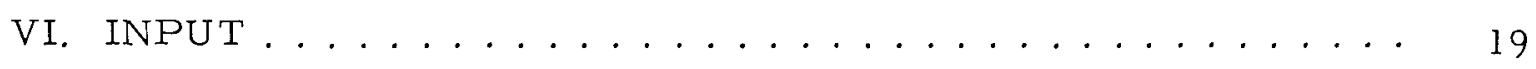

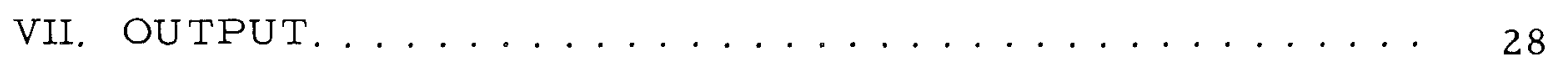

VIII. OPERATING INSTRUCTIONS ............... 30

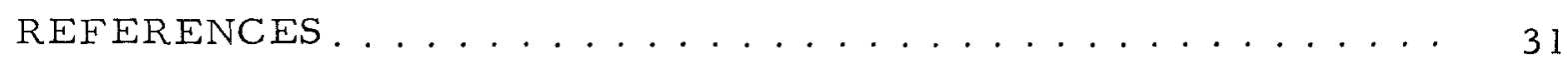

ACKNOW LEDGMENT . . . . . . . . . . . . . . . . . 32

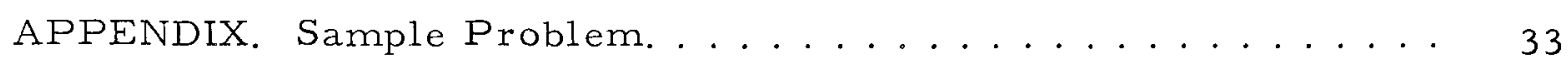




\title{
ELMOE: AN IBM-704 PROGRAM \\ TREATING ELASTIC SCATTERING RESONANCES \\ IN FAST REACTORS
}

by

A. L. Rago and H. H. Hummel

\begin{abstract}
A description of the operations carried out by the ELMOE code is given. This code carries out MUFT-type calculations for dealing with the elastic scattering resonances of the light elements present in fast reactors. Sources of information for the cross-section library in current use with the program, input specification, and operating instructions are also given.
\end{abstract}

\section{INTRODUCTION}

Elastic scattering resonances in the light elements present in fast power reactors have always been troublesome since they cause rapid fluctuations in the scattering cross sections of these materials, whereas variations of cross sections of fissile and fertile materials in the fast region are comparatively slow. The change in the neutron leakage and flux caused by adding or removing the light materials is difficult to calculate exactly, because the elastic moderation introduced by these materials causes perturbation of the neutron energy spectrum in the vicinity of the resonances.

In order to deal more effectively with problems of this type, an IBM-704 code, ELMOE, has been prepared. This code calculates the Fourier transform of the neutron flux for a mixture of moderators heavier than hydrogen, with the moderator-scattering law rigorously taken into account. Approximations available in the flux calculations are the usual P-1 and "consistent" P-1 and B-1 [1-3]. This code thus performs approximately the same calculation for moderators heavier than hydrogen as that which the MUFT codes [4] carry out for hydrogen. Although designed specifically for fast-reactor calculations, it can also be applied to thermal and intermediate reactors. No special provisions have yet been incorporated for dealing with capture resonances, however. Applications of the program have been previously reported [5-7]. 
A feature of the code is that certain parameters which are slowly varying need be specified only for "coarse" groups which are much wider in energy than the "fine" groups used to take detailed account of elastic moderation. This greatly reduces the amount of input data required.

The coarse groups are those ordinarily used in fast-reactor calculations with a total of 10 to 20 groups spanning the fast region. A finegroup structure is superimposed over all or a part of the usual groups. Associated with each of the fine groups is a set of Legendre polynomial expansion coefficients of the scattering cross section of each element. The original intention was that the fine-group network be fine enough to trace out the detailed variation of each scattering cross section. This has proved practicable except for the high-resolution data of Hibdon [8-10] for scattering from sodium, which are being specially handled as described later. From the Legendre polynomial expansion coefficients, elastictransfer, transport, and elastic-removal cross sections for the light elements are calculated for each fine group. Fission, capture, and inelastic-removal cross sections are normally kept constant for the fine groups corresponding to a given coarse group. Variable capture cross sections for the fine groups may be supplied if desired. Inelastic transfers entering the fine groups are distributed inproportion to the fine-group energy widths. The fission source has an energy dependence of the form $\sqrt{E} e^{-E} / T$ within the coarse groups. A variable fission source for the fine groups may be supplied if desired. No provision is made for inelastic transfers within coarse groups.

The fine groups all have the same lethargy width. Meshing of the coarse and fine groups is accomplished by a slight redefinition of the coarse-group energy limits. Although the fine-group lethargy width is arbitrary, in practice the labor involved in changing it except by integral multiples is such that only one width (and in some cases twice this width) has so far been used.

At least one coarse group at the top and bottom of the energy range must be handled in the usual way, i.e., not subdivided. Otherwise the finegroup region may cover any desired part of the energy spectrum. The groups not subdivided are calculated according to the usual equations for the Fourier transform. 


\section{CALCULATION OF CROSS SECTIONS}

1. Elastic-scattering Cross Sections

It is assumed in the calculation that data on the angular dependence of the elastic-scattering cross sections in the center-of-mass system are presented in the form

$$
\sigma_{s c}\left(\omega^{\prime}\right)=\sum_{l} B_{l} P_{l}\left(\mu^{\prime}\right)
$$

where

$$
\begin{aligned}
\sigma_{\mathrm{sc}}\left(\omega^{\prime}\right)= & \text { cross-section scattering into the solid angle } \\
& \text { element } \mathrm{d} \omega^{\prime}, \\
\mu^{\prime}= & \text { cosine of the scattering angle in the center-of-mass } \\
& \text { system, } \\
\mathrm{P}_{\ell}= & \ell^{\text {th }} \text { Legendre polynomial. }
\end{aligned}
$$

Data in the laboratory system are converted to the center-of-mass system. In the laboratory system the fractional energy change per collision is related to $\mu^{\prime}$ by [1].

$$
\frac{E}{E_{0}}=\frac{1}{(1+A)^{2}}\left(A^{2}+1+2 A \mu^{\prime}\right),
$$

or

$$
\mu^{\prime}=1-\frac{2}{\alpha}\left(1-\frac{E}{E_{0}}\right),
$$

where

$$
\begin{aligned}
\alpha & =4 \mathrm{~A} /(\mathrm{A}+1)^{2}, \\
\mathrm{~A} & =\text { moderator mass number, } \\
\mathrm{E}_{0} & =\text { initial energy of the neutron. }
\end{aligned}
$$

Integrating the scattering cross section over azimuth angle, one obtains

$$
2 \pi \sigma_{\mathbf{S C}}\left(\omega^{\prime}\right) \mathrm{d} \mu^{\prime}=\sigma_{\mathrm{SC}}\left(\mathrm{E}_{0} \rightarrow \mathrm{E}\right) \mathrm{dE} .
$$

Upon differentiating Eq. (II-2) and substituting this in Eq. (II-3) we obtain 


$$
\sigma_{S C}\left(E_{0} \rightarrow E\right) d E=\frac{4 \pi}{E_{0} \alpha} d E \sum_{\ell} B_{\ell} P_{\ell}\left(\mu^{\prime}\right)
$$

Let

$$
x=\frac{1}{\alpha}\left(1-\frac{E}{E_{0}}\right)
$$

Then, combining Eqs. (II-2) and (II-4), one may evaluate the Legendre polynomials in terms of:

$$
\begin{aligned}
& P_{1}\left(\mu^{\prime}\right)=\mu^{\prime}=1-2 x ; \\
& P_{2}\left(\mu^{\prime}\right)=\frac{1}{2}\left(3 \mu^{\prime 2}-1\right)=1-6 x+6 x^{2} ; \\
& P_{3}\left(\mu^{\prime}\right)=\frac{1}{2}\left(5 \mu^{\prime 3}-3 \mu^{\prime}\right)=1-12 x+30 x^{2}-20 x^{3} ; \\
& P_{4}\left(\mu^{\prime}\right)=\frac{1}{8}\left(35 \mu^{\prime 4}-30 \mu^{\prime 2}+3\right)=1-20 x+90 x^{2}-140 x^{3}+70 x^{4} .
\end{aligned}
$$

Therefore, $x$ varies from 0 to $l$ as $E$ varies from $E_{0}$, the initial energy, to $E=(1-\alpha) E_{0}$, the minimum possible final energy. This range is divided into $\mathrm{n}$ intervals chosen so that the ratio $\mathrm{r}$ of lower to upper energy for the interval is the same for each interval, i.e.,

$$
r=E_{j} /\left(E_{j-1}\right) .
$$

Therefore

$$
\begin{aligned}
\mathrm{x}_{0} & =0 ; \\
\mathrm{x}_{1} & =\frac{1}{\alpha}(1-\mathrm{r}) ; \\
\mathrm{x}_{2} & =\frac{1}{\alpha}\left(1-\mathrm{r}^{2}\right) ; \\
& \cdot \\
& \cdot \\
\mathrm{x}_{\mathrm{n}} & =\frac{1}{\alpha}\left(1-\mathrm{r}^{\mathrm{n}}\right)=1 ; \\
\mathrm{r} & =(1-\alpha)^{1 / n} .
\end{aligned}
$$

If one now integrates Eq. (II-4) over the final energy interval $\left(\mathrm{E}_{\ell}, \mathrm{E}_{\ell-1}\right)$ corresponding to the $\mathrm{x}$-interval $\left(\mathrm{x}_{\ell-1}, \mathrm{x}_{\ell}\right)$, 


$$
\int_{E_{\ell}}^{E_{\ell-1}} \sigma_{S C}\left(E_{0} \rightarrow E\right) d E=-4 \pi \int_{x_{\ell-1}}^{x_{\ell}} \mathrm{dx}\left[\sum_{\ell=0}^{4} B_{\ell} P_{\ell}\left(\mu^{\prime}\right)\right]
$$

Collecting terms $x_{\ell}^{i+1}$ and denoting the coefficients as $A_{i}$,

$\underset{\sigma_{e l t r}}{E_{0}{ }^{\ell}}=4 \pi\left[A_{0}^{0}\left(x_{\ell}-x_{\ell-1}\right)+A_{1}^{0} \frac{\left(x_{\ell}^{2}-x_{\ell-1}^{2}\right)}{2}+A_{2}^{0} \frac{\left(x_{\ell}^{3}-x_{\ell-1}^{3}\right)}{3}+A_{3}^{0} \frac{\left(x_{\ell}^{4}-x_{\ell-1}^{4}\right)}{4}+A_{4}^{0} \frac{\left(x_{\ell}^{5}-x_{\ell-1}^{5}\right)}{5}\right]$,

where

$$
\begin{aligned}
& \begin{array}{ll}
\begin{array}{l}
E_{0} \rightarrow \ell \\
\text { eltr }
\end{array} \quad \text { microscopic elastic-scattering cross section from } \\
\text { energy } E_{0} \text { to Group } l ;
\end{array} \\
& B_{\ell}^{0}=\text { coefficients of the Legendre polynomial expansion at energy } \\
& \quad E_{0} ; \\
& A_{0}^{0}=B_{0}^{0}+B_{1}^{0}+B_{2}^{0}+B_{4}^{0} \\
& A_{1}^{0}=2 B_{1}^{0}-6 B_{2}^{0}-12 B_{3}^{0}-20 B_{4}^{0} ; \\
& A_{2}^{0}=6 B_{2}^{0}+30 B_{3}^{0}+90 B_{4}^{0} ; \\
& A_{3}^{0}=-20 B_{3}^{0}-140 B_{4}^{0} ; \\
& A_{4}^{0}=70 B_{4}^{0} .
\end{aligned}
$$

If one now lets $E_{0}$ range over $E_{j}, j=0, \ldots, J$, the entire range under consideration, and $\ell$ range over the interval $\mathrm{k}=1, \ldots, \mathrm{J}$, where

$$
k=j+\ell,
$$

and

$$
E_{j}=\text { lower energy limit of Group } j,
$$

then, for the general case,

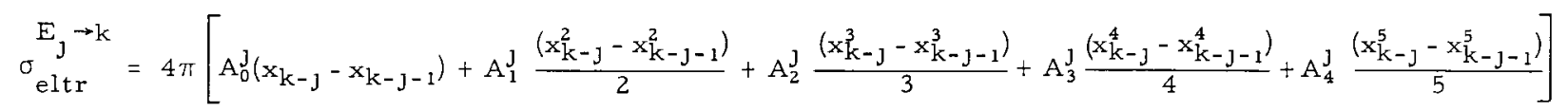

The elastic-scattering cross section from Group $j$ to Group $k$ is calculated from 


$$
\sigma_{\text {eltr }}^{j \rightarrow k}=\frac{\sigma_{j \rightarrow k}+\sigma_{j-l \rightarrow k}}{2}
$$

\section{Elastic-removal Cross Sections}

The elastic-removal cross section from energy $E_{j}$ to below $E_{j+1}$ may be represented as

$$
\sigma_{e l r}^{E_{j}, E_{j+1}}=\sum_{k=j+2}^{j+n} \underset{\sigma j}{E_{j} \rightarrow k}
$$

Substituting from Eq. II-6 we get

$$
\sigma_{\mathrm{elr}}^{E_{j}, E_{j+1}}=4 \pi\left[A_{0}^{j}\left(1-x_{1}\right)+A_{1}^{j} \frac{\left(1-x_{1}^{2}\right)}{2}+A_{2}^{j} \frac{\left(1-x_{1}^{3}\right)}{3}+A_{3}^{j} \frac{\left(1-x_{1}^{4}\right)}{4}+A_{4}^{j} \frac{\left(1-x_{1}^{5}\right)}{5}\right] \text {. }
$$

The elastic-removal cross section from Group $j$ is calculated as

$$
\sigma_{\mathrm{e} 1 \mathrm{r}}^{\mathrm{j}}=\frac{1}{2}\left({ }_{\sigma \mathrm{elr}}^{\mathrm{E}_{\mathrm{j}-1^{\mathrm{E}} \mathrm{j}}+4 \pi \mathrm{B}_{0}^{\mathrm{j}}}\right) \text {. }
$$

3. Transport Cross Sections

The transport cross section is given by

$$
3 \sigma_{\mathrm{tr}}^{\mathrm{E}_{\mathrm{j}}}=3 \sigma_{\mathrm{sc}}^{\mathrm{E}_{\mathrm{j}}}(1-\bar{\mu})+3 \sigma_{\mathrm{a}}
$$

where

$$
\begin{aligned}
\mu= & \text { average scattering angle cosine in laboratory system, } \\
\sigma_{a}= & \text { total removal cross section due to processes other than } \\
& \text { elastic scattering. } \\
\sigma_{\mathrm{sc}} & =4 \pi \mathrm{B}_{0}^{\mathrm{j}} .
\end{aligned}
$$

From Eq. (II-8), 
$3 \sigma_{t r}^{E_{j}}=12 \pi B_{0}^{j}\left(1-\frac{2}{3 A}-u_{1,1}^{-1} \frac{B_{1}^{j}}{3 B_{0}^{j}}-u_{1,2}^{-1} \frac{B_{2}^{j}}{3 B_{0}^{j}}-u_{1,3}^{-1} \frac{B_{3}^{j}}{3 B_{0}^{j}}-u_{1,4}^{-1} \frac{B_{4}^{j}}{3 B_{0}^{j}}\right)+3 \sigma_{a}$,

or

$3 \sigma_{\operatorname{tr}}^{E_{j}}=4 \pi\left(3 B_{0}^{j}-\frac{2 B_{0}^{j}}{A}-u_{1,1}^{-1} B_{1}^{j}-u_{1,2}^{-1} B_{2}^{j}-u_{1,3}^{-1} B_{3}^{j}-u_{1,4}^{-1} B_{4}^{j}\right)+3 \sigma_{a}$,

where $u_{1,1}^{-1}, u_{1,2}^{-1}, u_{1,3}^{-1}$ and $u_{1,4}^{-1}$ are elements of the transformation matrix $\mathrm{U}^{-1}[1]$ transforming the $\mathrm{B}_{\ell}$ from the center-of-mass system to the laboratory system. As above, the cross section at Group $j$ is calculated by

$$
3 \sigma_{t r}^{j}=\frac{3 \sigma_{t r}^{E_{j}}+3 \sigma_{t r}^{E_{j-1}}}{2}+3 \sigma_{a} \text {. }
$$




\section{FLUX APPROXIMATIONS}

There are four flux approximations available as options in ELMOE. The equations are given below.

\section{P-1 Approximation}

For the P-l approximation, the equation for the flux in the $k^{\text {th }}$ group is

$$
\phi_{\mathrm{k}}^{i}=\frac{\beta_{\mathrm{k}}+\psi_{\mathrm{k}}}{\frac{\mathrm{B}_{\mathrm{i}}^{2}}{3 \sum_{\mathrm{tr}}^{\mathrm{k}}}+\Sigma_{\mathrm{a}}^{\mathrm{k}}+\Sigma_{\mathrm{elr}}^{\mathrm{k}}},
$$

where

$$
\begin{aligned}
\phi_{k}^{i}= & i^{\text {th }} \text { iterate of the flux group } k ; \\
\beta_{k}= & \text { fraction of fission source in group } k \text { (assumed same for } \\
& \text { all isotopes); } \\
\psi_{k}= & \sum_{j=1}^{k-1}\left(\sum_{\text {eltr }}^{j \rightarrow k}+\sum_{i n t r}^{j \rightarrow k}\right) \phi j=\text { scattering source from higher } \\
& \text { energies; } \\
\sum_{\text {j }}^{j \rightarrow k}= & \text { elastic-scattering transfer cross section, group } j \text { to } \\
& \text { group } k ; \\
\sum_{j \rightarrow k}^{j \rightarrow k}= & \text { inelastic-scattering transfer cross section, group } j \text { to } \\
& \text { group } k ; \\
\sum_{a}^{k}= & \text { absorption (total non-elastic removal) cross section for } \\
& \text { group } k ; \\
\sum_{e l r}^{k}= & \text { elastic-removal cross section for group } k ; \\
\sum_{t r}^{k}= & \text { transport cross section for group } k ; \\
B_{i}^{2}= & \text { the ith iterate of the buckling. }
\end{aligned}
$$

This equation is calculated by iteration until convergence on $B^{2}$, which is decided by

$$
\left|\sum_{k=1}^{N} \nu_{k} \Sigma_{k}^{f} \phi_{k}^{i}-1\right| \leq \epsilon,
$$

where

$$
\begin{aligned}
\nu_{k} \sum_{k}^{f} & =\text { neutrons per fission times fission cross section for group } \mathrm{k}, \\
\epsilon & \text { and } \\
& \text { convergence criterion for iterative scheme. }
\end{aligned}
$$


In the further discussion, the superscript "1" is dropped as a convergence is assumed

\section{B-1 Approximation}

If one now replaces $\Sigma_{\mathrm{tr}}^{\mathrm{k}}$ in Eq (III-1) by $\gamma_{\mathrm{k}} \Sigma_{\mathrm{tr}}^{\mathrm{k}}$, where $\gamma_{\mathrm{k}}$ 1s defined as

$$
\gamma_{k}=\frac{\alpha_{k} \tan ^{-1} \alpha_{k}}{3\left(1-\frac{\tan ^{-1} \alpha_{k}}{\alpha_{k}}\right)}, \quad \alpha_{k}=\frac{B}{\sum_{t r}^{k}},
$$

one obtains the B-1 approximation [2] Under this transformation, Eq (III-1) becomes

$$
\phi_{\mathrm{k}}=\frac{\beta_{\mathrm{k}}+\psi_{\mathrm{k}}}{\frac{\mathrm{B}^{2}}{3 \gamma_{\mathrm{k}} \sum_{\mathrm{tr}}^{\mathrm{k}}}+\Sigma_{\mathrm{a}}^{\mathrm{k}}+\Sigma_{\mathrm{elr}}^{\mathrm{k}}},
$$

and the iterative procedure used in the $\mathrm{P}-1$ approximation is used for solution

3 "Consistent" P-1 Approximation

In the ordinary P-l approximation, the angular distribution of the slowing-down source is not properly taken into account The equations correctly taking it into account in the P-1 approximation are given in [1] as Eqs ( $1131 \mathrm{a})$ and ( $113 \mathrm{lb})$ For simplicity, the se are given here with spatial variation in the $Z$ direction only assumed

$$
\begin{aligned}
& \frac{1}{4 \pi} \frac{\partial J(E, Z)}{\partial Z}=\int_{0}^{\infty} \phi\left(E^{\prime}, Z\right) S_{0}\left(E^{\prime} \rightarrow E\right) d E^{\prime}-\frac{\sum_{\text {tot }}(E) \phi(E, Z)}{4 \pi}+S_{00}(E, Z) \\
& \frac{1}{4 \pi} \frac{\partial \phi(E, Z)}{\partial Z}=\int_{0}^{\infty} J\left(E^{\prime}, Z\right) S_{1}\left(E^{\prime} \rightarrow E\right) d E^{\prime}-\frac{3 \Sigma_{\text {tot }}(E) J(E, Z)}{4 \pi}, \\
& J(E, Z)=\text { current, } \\
& \phi(E, Z)= \text { flux, } \\
& 4 \pi S_{00}(E Z)=\text { inelastic-scattering plus fission sources (assumed } \\
& \text { isotropic) }
\end{aligned}
$$

For the Fourier transform one can set

$$
J(E, Z)=-1 J(E) e^{1 B Z}
$$




$$
\phi(E, Z)=\Phi(E) e^{i B Z},
$$

and

$$
S_{00}(E, Z)=S_{00}(E) e^{i B Z} .
$$

In multigroup notation, with $E^{\prime}$ and $E$ corresponding to groups $j$ and $k$, respectively,

$$
S_{0}\left(E^{\prime} \rightarrow E\right) \rightarrow S_{0}^{j \rightarrow k}=\frac{1}{4 \pi} \sum_{\text {eltr }}^{j \rightarrow k}
$$

and

$$
S_{1}\left(E^{\prime} \rightarrow E\right) \rightarrow S_{1}^{j \rightarrow k}=-\frac{1}{4 \pi} \frac{3}{2}\left(\frac{E_{k}}{E_{j}}\right)^{1 / 2}\left[(A+1)-\frac{(A-1)}{E_{k} / E_{j}}\right] \sum_{e l t r}^{j \rightarrow k}
$$

$E_{j}$ and $E_{k}$ may be taken as the lower group limits:

$$
\begin{aligned}
& J(E) \rightarrow J_{k}, \\
& \phi(E) \rightarrow \phi_{k} .
\end{aligned}
$$

Writing Eq. (III-3) in multigroup form with the use of Eq. (III-5),

$$
B J_{k}=\sum_{j=k-n}^{k-1} \phi_{j}\left[\begin{array}{c}
\sum_{\text {eltr }}^{j \rightarrow k}+\sum_{i n t r}^{j \rightarrow k} \\
\text { iln }
\end{array}\right]-\left(\sum_{a}^{k}+\Sigma_{e l r}^{k}\right) \phi_{k}+\beta_{k} .
$$

Equation (III-4) can be similarly rewritten as

$$
\frac{J_{k}}{B}=\frac{\phi_{k}}{3 \sum_{t o t}^{k}}+\frac{1}{3 \sum_{t o t}^{k}} \sum_{j=k-n}^{k-1} \frac{J_{j}}{B} \frac{3}{2}\left(\frac{E_{k}}{E_{j}}\right)^{1 / 2}\left[(A+1)-\frac{(A-1)}{E_{k} / E_{j}}\right] \sum_{\text {eltr }}^{j \rightarrow k},
$$

and we define

$\sum_{\mathrm{tot}}^{\mathrm{k}}=\sum_{\mathrm{tr}}^{\mathrm{k}}+\bar{\mu}_{\mathrm{k}} \Sigma_{\mathrm{elr}}^{\mathrm{k}}$.

Solving Eqs. (III-6) and (III-7) for the flux, one obtains

$$
\phi_{k}=\frac{\beta_{k}+\sum_{j=k-n}^{k-1} \phi_{j}\left[\Sigma_{\mathbf{e l t r}}^{j} \vec{k}+\sum_{i n t r}^{j \rightarrow k}\right]-\frac{1}{3 \Sigma_{t o t}^{k}} \sum_{j=k-n}^{k-1} \frac{3}{2}\left(\frac{E_{k}}{E_{j}}\right)^{1 / 2} B J_{j}\left[(A+1)-\frac{(A-1)}{E_{k} / E_{j}}\right] \Sigma_{e l t r}^{j \rightarrow k}}{\frac{B^{2}}{3 \Sigma_{\text {tot }}^{k}}+\Sigma_{a}^{k}+\Sigma_{\text {elr }}^{k}}
$$


Equations (III-7) and (III-8) are a coupled set which are solved iteratively to obtain the group fluxes and currents, and the eigenvalue $B^{2}$.

4. "Consistent" B-1 Approximation[2, 3]

If one substitutes $\gamma_{k} \sum_{\text {tot }}^{k}$ for $\sum_{\text {tot }}^{k}$, where $\gamma_{k}$ is defined as

$$
\gamma_{k}=\frac{\alpha_{k} \tan ^{-1} \alpha_{k}}{3\left(1-\frac{\tan ^{-1} \alpha_{k}}{\alpha_{k}}\right)} ; \quad \alpha_{k}=\frac{B}{\sum_{\text {tot }}^{\mathrm{k}}} \text {, }
$$

one obtains the "consistent" B-l approximation, in which a rigorous solution for the Fourier transform is obtained except that only a P-1 expansion of the scattering cross sections in the laboratory system is used. For this case, Eqs. (III-7) and (III-8) are transformed to

$$
\frac{J_{k}}{B}=\frac{\phi_{k}}{3 \gamma_{k} \sum_{t o t}^{k}}+\frac{1}{3 \gamma_{k} \sum_{\text {tot }}^{k}} \sum_{j=k-n}^{k-1} \frac{J_{j}}{B} \frac{3}{2}\left(\frac{E_{k}}{E_{j}}\right)^{1 / 2}\left[(A+1)-\frac{(A-1)}{E_{k} / E_{j}}\right] \sum_{\text {eltr }}^{j \rightarrow k}
$$

and

$$
\phi_{k}=\frac{\beta_{k}+\sum_{j=k-n}^{k-1} \phi_{j}\left[\sum \sum_{\text {eltr }}^{j+k}+\Sigma_{1 n t r}^{j-k}\right]-\frac{1}{3 \gamma \sum_{\text {tot }}^{k}} \sum_{j=k-n}^{k-1} \frac{3}{2}\left(\frac{E_{k}}{E_{j}}\right)^{1 / 2} B J_{j}\left[(A+1)-\frac{(A-1)}{E_{k} / E_{j}}\right] \sum_{\text {eltr }}^{j \rightarrow k}}{\frac{B^{2}}{3 \gamma_{k} \sum_{\text {tot }}^{k}}+\sum_{a}^{k}+\Sigma_{\text {elr }}^{k}}
$$

Again, the solution involves coupling Eqs. (III-10) and (III-11), with iteration on $B^{2}$.

For each of these approximations when the convergence criterion is satisfied, the code calculates a flux-weighted average of the fine-group cross sections to get an average homogenized coarse-group cross-section set. The coarse-group transport cross section is averaged for each of the options as follows:

$$
\begin{array}{lc}
\frac{\frac{P-1}{\left(\frac{1}{3 \Sigma_{t r}}\right)}=\frac{\sum \phi / 3 \Sigma_{t r}}{\sum \phi}}{\sum} & \bar{\Sigma}_{t r}-\frac{B}{\tan ^{-1} \frac{B}{\bar{\Sigma}_{t r}}}+\bar{D} B^{2}=0 \\
\frac{B-1 \text { and Consistent B-1 }}{\sum \phi} & \text { for } \bar{\Sigma}_{t r} .
\end{array}
$$


Consistent $P_{\infty} 1$

$\bar{D}=\frac{1}{\overline{3 \Sigma}_{\operatorname{tr}}}=\frac{\sum J / B}{\sum \phi}$.

These averaged cross sections are then used to do a normal fundamental mode calculation, using diffusion theory for the $\mathrm{P}-1$ and Consistent $\mathrm{P}-1$ cases, and the asymptotic solution to the transport equation for the B-1 and Consistent B-1 cases. 


\section{TREATMENT OF NARROW RESONANCES}

The ratio of final to initial fine-group energies thus far being used is 0.99133 , so that at $100 \mathrm{keV}$ the group width is $0.87 \mathrm{keV}$ and at $200 \mathrm{keV}$ 1.74. Since many of the resonances in sodium reported by Hibdon [8] in this energy region have widths less than $1 \mathrm{keV}$, this group spacing cannot be used to describe such resonance shapes. The method of dealing with these very narrow resonances is as follows. First, a problem is run with the low-resolution data for sodium given in [12], which can be adequately traced out with the group spacing being used. The usual assumption of the narrowresonance approximation is made: that over one of the fine groups the total source is constant and equal to that calculated with the low-resolution data. For the P-l approximation the actual magnitude of the source for each group need not be known. In this case the assumption is made that the source $S$ equals $\phi(E) \Sigma_{\text {tot }}(E)$ within the fine group. An average transport cross section for fine groups is obtained from

$$
\frac{l}{\Sigma_{t r}^{j}}=\frac{\int_{E_{j-1}}^{E_{j}} \frac{d E}{\sum_{t o t}(E) \Sigma_{t r}(E)}}{\int_{E_{j-1}}^{E_{j}} \frac{d E}{\Sigma_{t o t}(E)}} .
$$

$\Sigma_{\text {tot }}(E)$ and $\Sigma_{\text {tr }}(E)$ are obtained from the detailed sodium data, with the contributions of material other than sodium being held at the values for the original ELMOE claculation. Finally, the values of $1 / \sum_{\mathrm{tr}}^{j}$ are weighted with the fluxes from the ELMOE calculation to obtain a revised coarsegroup value.

To obtain a corrected scattering cross section for elastic-removal calculation, the following integral is computed:

$$
\sigma_{S N a}(j)=\frac{\int_{E_{j-1}}^{E_{j}} \frac{\sigma_{S N a}(E) d E}{\Sigma_{t o t}(E)}}{\int_{E_{j-1}}^{E_{j}} \frac{d E}{\sum_{t o t}(E)}}
$$

These values of $\sigma_{\mathrm{SNa}}(\mathrm{j})$ are used in conjunction with the original ELMOE fluxes to obtain a corrected elastic-removal cross section for sodium. A library tape containing $\sigma_{\mathrm{SNa}}(\mathrm{E})$ is available for this calculation. 


\section{LIBRARY TAPE}

As mentioned above, the elastic-scattering-matrix calculation uses the coefficients $B_{L}$ in the expression

$$
\sigma\left(\theta_{C}\right)=\sum_{L} B_{L} P_{L}\left(\cos \theta_{C}\right),
$$

where $\sigma\left(\theta_{C}\right)$ is the differential scattering cross section per unit solid angle in the center-of-mass system. The coefficients $B_{L}$ are supplied to the program by means of a library tape. The first file of the library tape contains the multigroup cross-section sets. The coefficients BL comprise the second file. The multigroup cross-section sets are described below (Section VI).

The coefficients may be supplied in either laboratory or centerof-mass system, and the ratio of the coefficient to the $L=0$ coefficient may be supplied if desired. All coefficients are converted to be stored in the center-of-mass system on the library tape.

The library currently contains coefficients for the following isotopes: carbon, oxygen, sodium, sodium-1, sodium-2, aluminum, vanadium, chromium, iron, Type 304 stainless steel, Hastelloy, nickel and niobium.

Sodium-1 coefficients were obtained by doing an ELMOE calculation for a typical metal core and then using the narrow-resonance option from $66 \mathrm{keV}$ to $830 \mathrm{keV}$, and using the re-averaged total scattering cross section for sodium output from this procedure as the basis for new values of $B_{0}$ for sodium- 1 in this range. The higher $B_{L}$ in this range were kept at the same ratio as for sodium. Sodium-2 coefficients were obtained in the same manner except that the ELMOE calculation was for an oxide core. Type 304 stainless steel coefficients are simply linear combinations of those for iron, chromium, and nickel with volume fractions by weight of $0.72,0.19$, and 0.09 respectively. Hastelloy coefficients were constructed in a similar manner, with the composition assumed to be 0.22 iron, 0.24 chromium, and 0.54 nickel, molybdenum being neglected. Total crosssection data were taken [12] and [13]. Total cross sections of sodium for the detailed treatment by the narrow-resonance approximation (Section IV) were taken from [8-10]. Since high-resolution angular-distribution data are available for only a few isolated resonances, the procedure followed was to derive the coefficient of the isotropic term from total cross-section data and to take the ratio of coefficients corresponding to higher values of $L$ to the $L=0$ coefficient from the angular-distribution data. Sources of angular-distribution and inelastic-scattering data were as follows: 


\section{Sodium}

In the energy range 200-800 keV the data of [14] were used. Outside this range up to $1400 \mathrm{keV},[15]$ was used. Above $1400 \mathrm{keV}, \omega_{1}$ was based on $\bar{\mu}=\omega_{1} / 3 \omega_{0}$ given in Fig. 22 of [16], where the $\omega$ 's are defined by

$$
\sigma_{\mathbf{s}}(\theta)=\frac{\sigma_{\mathbf{s}}}{4 \pi} \sum_{\mathrm{L}} \omega_{\mathrm{L}} \mathrm{P}_{\mathrm{L}}(\cos \theta),
$$

in which

$$
\begin{aligned}
\theta & =\text { scattering angle in the laboratory system, } \\
\sigma_{S} & =\text { total scattering cross section. }
\end{aligned}
$$

The quantities $\omega_{2}$ and $\omega_{3}$ were obtained by linear extrapolation to 1.49 and 0.61 , respectively, at $3.7 \mathrm{MeV}$. This was based on data in [17] for sulphur. Total scattering cross sections were obtained by subtracting the inelastic-scattering cross sections of [18] from the total cross-section data.

\section{$\underline{\text { Aluminum }}$}

The same procedure was followed in this case as for sodium, except that the only angular-distribution data available below $1400 \mathrm{keV}$ were, and are, those of [15]. Inelastic-scattering cross sections were taken from [19].

\section{Iron, Nicke1, Chromium}

The data of [15] were used up to $1800 \mathrm{keV}$ for $\omega_{1}, \omega_{2}, \omega_{3}$, and $\omega_{4}$. $\omega_{1}$ to $3.7 \mathrm{MeV}$ was obtained from $\bar{\mu}$ in [16]. $\omega_{2}, \omega_{3}$, and $\omega_{4}$ were obtained by linear extrapolation to $2.02,1.77$, and 1.10 , respectively, at $3.7 \mathrm{MeV}$, this point being based on the data in [17]. Inelastic-scattering cross sections were those of [19].

\section{Carbon}

In the energy range from 0 to $2.2 \mathrm{MeV}$ the data of [20] were used, whereas in the range from 2.2 to $3,7 \mathrm{MeV}$ the data of [21] were employed.

Oxygen

In the energy range from 0 to $1.7 \mathrm{MeV}$ the data of [20] were used, where above this to $3.7 \mathrm{MeV}$ the data in [22] were used. 
Niobium and Vanadium

In the range from 0 to $1.35 \mathrm{MeV}$ the data of [15] were used. Above $1.35 \mathrm{MeV}, \omega_{1}$ was based on $\bar{\mu}$ given in [16]. Coefficients for higher values of $\mathrm{L}$ have not yet been supplied above $1.35 \mathrm{MeV}$.

The available library of coefficients clearly leaves much to be desired. The energy resolution of the cross-section data, particularly of the angular-distribution data, is in general not nearly as good as one would like to have. The necessity of using angular-distribution data of resolution differing widely from the total cross-section data is not very satisfactory. In some cases, the total cross section obtained in the angular-distribution measurements is clearly inconsistent with the other total cross-section data from transmission measurements, even taking into account the difference of resolution. A better estimate of highenergy angular distribution than that made here could undoubtedly be obtained by means of the optical model of the nucleus. Since the library was prepared in 1960, it does not include data available later than that except for those of [9] and [10].

A library tape containing more detailed data for sodium due to Hibdon [8-10] is utilized by the narrow sodium-resonance routine. Energy vs. total scattering cross section for sodium are given for the range from 60 to $832 \mathrm{keV}$ in increments of 0.1 and $0.2 \mathrm{keV}$. 


\section{INPUT}

The coarse-group cross sections are taken from one of a library of such sets available. These may be either in the form used in the REX code [23] or in that of the SNG code [24]. Elastic-removal cross sections for the light elements used in these sets must be supplied, as they are subtracted out. The Legendre polynomial expansion coefficients for the light elements may be supplied either in the center-of-mass or the laboratory system. The library of such coefficients currently available includes the following elements: $\mathrm{Fe}, \mathrm{Ni}, \mathrm{Cr}, \mathrm{Nb}, \mathrm{V}, \mathrm{Al}, \mathrm{Na}, \mathrm{Na}-1, \mathrm{Na}-2, \mathrm{C}, \mathrm{O}$, stainless steel, and Hastelloy.

The number of fine groups that can be used is limited by the $32 \mathrm{~K}-$ core storage. If more groups than this limit are desired, the problem is run in more than one pass, subdividing different energy regions with an overlap of at least one or two coarse groups to produce a proper elastic moderation source at the upper end of the subdivided region. The number of fine groups that can be used is a function of the maximum size of the elastic scattering matrix. The Legendre coefficient library currently in use corresponds to elastic-scattering matrices of order 20, 29, and 39 for sodium, oxygen, and carbon, respectively. When sodium, oxygen, or carbon is the lightest element present, the maximum number of fine groups that can be used in the ordinary $P-1$ calculation is 880,682 , and 535 , respectively. With the consistent calculations, the corresponding values are 535,393 , and 303.

Materials in a problem that require Legendre coefficients for cross-section evaluation are referred to as "light" elements. Those that do not are referred to as "heavy." A feature of ELMOE allows problems to be done in "sets." A "set" is defined as a group of problems that differ only in composition (value of volume fraction). For the set only one calculation of the elastic-scattering matrix is executed, effecting a considerable time saving.

Description of the Input

ELMOE Calculation

\section{Card}

Set FORTRAN

No.

Symbol

Format

Definition

$1 \quad$ IDB

(13I4,E 12.5)

Identification of Legendre coefficient set required from Library Tape.

IDX

Identification of macroscopic cross section set required from Library Tape. 
Card

Set FORTRAN

No.

Symbol

Format

Definition

MEND

MTOT

JEND

$\mathrm{MH}$

KM2

KMIN

KEND

$\mathrm{IT}=1$

$\mathrm{KT}=1$

$\operatorname{ITH}=1$

NW 1

$\mathrm{EO}$

2

$\operatorname{KRSEC}(1), \operatorname{KRSEC}(2), \ldots$, KRSEC(MEND + MTOT)

3

KRSET(1), $\operatorname{KBSET}(2), \ldots$, KBSET(MTOT)
Number of heavy elements, $\leq 19$.

Number of light elements, $1 \leq$ MTOT $\leq 20, \mathrm{MTOT}+\mathrm{MEND} \leq 20$.

Number of fine groups. For limit see NWl.

Order of Legendre expansion used for differential scattering cross section, $\leq 5$.

If $\Delta$ is lethargy increment of Legendre library, problem lethargy increment is (KM2)A.

Initial coarse group subdivided.

Final coarse group subdivided.

Determines maximum size of scattering matrix (JEND $\times \mathrm{N}$ ) (See Card Set No. 5)

$\mathrm{NW} 1=1 \quad$ IOPT $=1,2 \quad(880 \times 20)$ $N W 1=2 \quad$ IOPT $=1,2 \quad(682 \times 29)$

$\mathrm{NWl}=3 \quad$ IOPT $=1,2 \quad(545 \times 39)$

$N W 1=4 \quad$ IOPT $=3,4 \quad(535 \times 20)$

$N W 1=5 \quad$ IOPT $=3,4 \quad(393 \times 29)$

$\mathrm{NWl}=6 \quad$ IOPT $=3,4 \quad(303 \times 39)$

Energy in $\mathrm{keV}$ of the top of group KMIN .

Indicates position in cross-section set of materials used in problem.

Indicates position in Legendre coefficient set of materials used in problem.

Problem identification. Column 1 blank, 2-72, alphanumeric

characters. 
Card

Set

FORTRAN

No.

Symbol

5
Format

IOPT
Flux approximation.

$\mathrm{IOPT}=1 \mathrm{P}-1$.

$\mathrm{IOPT}=2 \mathrm{~B}-1$.

$\mathrm{IOPT}=3$ "Consistent" $\mathrm{P}-1$.

IOPT $=4$ "Consistent" B-1.

Output options.

$I A=1$ Normal.

IA $=2$ Fine-group elastic-removal cross section printed out for each light material.

$I A=3$ Fine-group elastic-scattering matrix and elastic-removal cross section printed out for each light material.

IC

IC $=1$ Fine-group output omitted.

$I C=2$ Fine-group output printed .

Input options.

ID

IE

IF

NM

$\mathrm{N}$

NTIMES
ID $=1$ Fine-group capture cross section not supplied as input.

ID $=2$ Fine-group capture cross section supplied as input.

$I E=1$ Fine fission source not supplied as input.

$\mathrm{IE}=2$ Fine fission source supplied as input.

Preliminary sodium NR treatment option.

IF $=1$ Normal.

$I F=2$ Preliminary problems for sodium narrow-resonance treatment.

Position in problem of sodium.

Maximum energy loss per collision of sodium (in groups).

Number of problems in set. 
Card

Set FORTRAN

No. Symbol

6

Format

Definition

(18I4)

NUM(KMIN),

...,

NUM(KEND)

7

(6E 12.5)

$A(1), A C(1)$,

$U_{11}^{-1}(1), U_{12}^{-1}(1)$,

$\mathrm{U}_{13}^{-1}(1), \mathrm{U}_{14}^{-1}(1)$,

$\cdots$

$\mathrm{A}(\mathrm{MTOT})$,

$\mathrm{AC}(\mathrm{MTOT})$,

$\mathrm{U}_{11}^{-1}(\mathrm{MTOT})$,

$\mathrm{U}_{12}^{-1}$ (MTOT),

$\mathrm{U}_{13}^{-1}(\mathrm{MTOT})$,

$\mathrm{U}_{14}^{-1}(\mathrm{MTOT})$

$\mathrm{A}(\mathrm{MTOT}+1)$,

$\mathrm{AC}(\mathrm{MTOT}+1)$

... ,

A(MTOT+MEND),

AC(MTOT+MEND)

8

(6E 12.5)

EPS, BBO, V(1),

$\cdots$

$\mathrm{V}(\mathrm{MTOT}+\mathrm{MEND})$

9 If $I E=2$

$\mathrm{KU}, \mathrm{KL}, \beta(\mathrm{KU})$,

$\ldots$,

$\beta(\mathrm{KL})$

10 If ID $=2$

LL

$(2 \mathrm{I} 4 /(6 \mathrm{E} 12.5))$

Number of fine groups in standard groups subdivided.

Atomic weight, atoms/cc, transformation vector [11] from CMS to LAB system for material 1.

Atomic weight, atoms/cc, trans formation vector from CMS to LAB system for last light material.

Atomic weight and atoms/cc for first heavy material.

Atomic weight and atoms/cc for last heavy material.

Convergence criteria, initial buckling guess, volume fractions for material 1 through MTOT+ MEND.

Fine-group limits of specification, followed by the fission source for these fine groups.

Number of batches of crosssection input.

(4I4/(6E 12.5))

$\mathrm{M}^{1}, \mathrm{~K}^{1}, \mathrm{KU}^{1}, \mathrm{KL}^{1}$,

$\sigma_{\mathrm{C}}^{1}(\mathrm{KU})$,

$\ddot{\sigma}_{\mathrm{C}}^{1}(\mathrm{KL})$

Material number (position in problem), coarse group, finegroup limits within coarse group, and capture cross sections for batch 1 . 


\begin{tabular}{|c|c|c|c|}
\hline $\begin{array}{l}\text { Card } \\
\text { Set } \\
\text { No. }\end{array}$ & $\begin{array}{c}\text { FORTRAN } \\
\text { Symbol }\end{array}$ & Eormat & Definition \\
\hline 10 & & $(4 \mathrm{I} 4 /(6 \mathrm{E} 12.5))$ & \\
\hline & $\begin{array}{l}M^{L L}, K^{L L}, K^{L L}, K L L L \\
\sigma_{C}^{L L}(K U), \ldots, \sigma \frac{L}{L}(K L)\end{array}$ & & $\begin{array}{l}\text { Material number, coarse } \\
\text { group, fine-group limits } \\
\text { within coarse group, and } \\
\text { capture cross sections for } \\
\text { batch LL. }\end{array}$ \\
\hline 11 & If NTIMES > & & $\begin{array}{l}\text { Repeat card set numbers } 4 \\
\text { and } 8-10 \text { for each problem } \\
\text { in set. }\end{array}$ \\
\hline
\end{tabular}

Note: If attempting to iterate on the buckling with a non-multiplying medium, a negative input buckling guess must be given.

Treatment for Sodium Narrow Resonances

Card

$\begin{array}{lll}\begin{array}{c}\text { Set } \\ \text { No. }\end{array} & \begin{array}{c}\text { FORTRAN } \\ \text { Symbol }\end{array} & \text { Format } \\ 1 & \text { blank card } & \\ 3 & \text { ID } & (72 \mathrm{X}) \\ & & (3 I 4)\end{array}$

KMIN

First-group narrowresonance treatment is performed.

KEND

Last-group narrowresonance treatment is performed.

$\mathrm{KD}$

Output option.
$\mathrm{KD}=1$ Fine-group-averaged total scattering and transport cross sec- tion printed out.
$\mathrm{KD}=2$ Fine-group-averaged total scattering and transport cross sec- tion not printed out.

Note: Problems run on the sodium narrow-resonance routine may be run only one at a time, i.e., not more than one to a set. 
To Add Cross-section Sets to Library Tape

Card

Set FORTRAN

No. Symbol Format

Definition

1

IDB

$=0$

IDX

non-zero

2

KNUM

$=0$ Add to existing tape.

$=1$ Start new tape.

IDNUM

Cross-section set identification.

$M E$

Number of materials in cross-section set, $\leq 20$.

KAN

Number of inelastic-scattering groups, $\leq 23$.

KMAX

Number of energy groups, $\leq 24$.

IRS

Cross-section format option

$=1$ REX cross-section format.

$=2$ SN cross-section format.

KSET

Number of cross-section sets being added to tape at this time.

3 Cross-section deck

The SN cross-section cards contain eight words per card with a blank column between words. Column one always contains a nine punch and the sign of the number is placed over the "one-tenth's" digit, thereby designating the position of the decimal point. The cross sections are written in the following order:
Col. 1
Col. $2-9$
Col. $11-18$
Col. $20-27$
Col. 29-36
Col. $38-45$
9 punch
$\nu \sigma_{f, j}$
$\sigma_{t r, j}$
$\sigma_{t r, j}-\sigma_{a, j}$
$\sigma_{\text {in }}(j-1 \rightarrow j)$
$\sigma_{\text {in }}(j-2 \rightarrow j)$
$\sigma_{\text {in }}(j-L \rightarrow j)$

where $L=$ total number of inelastic-scattering groups for the cross-section set and $j=1,2, \ldots, J$ (the group index). The cards are arranged groupwise for each material and the fission spec$\operatorname{trum}\left(\beta_{j}\right)$ precedes the cross-section data. 


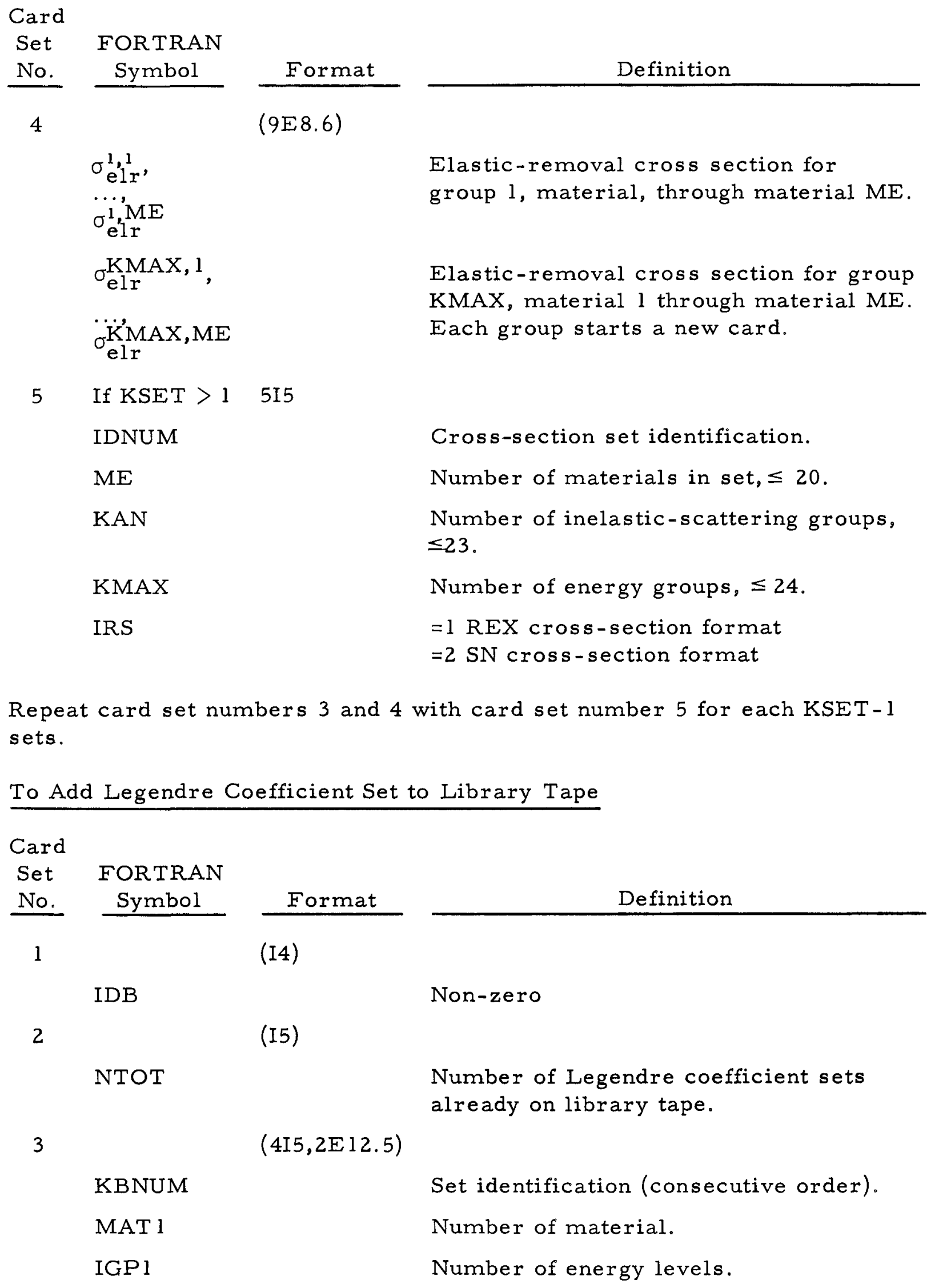


Card

Set

FORTRAN

No.

Symbol

Format

Definition

3

MADP

$=1$ Add complete set.

$=2$ Add MAT I materials to set KBNUM.

$=3$ Add IGPl energy level following existing levels to set KBNUM.

$=4$ Add IGPl energy levels preceding existing levels of set KBNUM.

EEOl

Energy of first level being added to set KBNUM.

U I

Constant energy ratio of set, $E_{i} / E_{i=1^{\circ}}$ 。

4 $(6 E 12.5)$
$A(1)$,
$\cdots$,
$\mathrm{A}(\mathrm{MAT} 1)$

5

$\operatorname{IB}^{1}, \operatorname{IW}^{1}(1), \operatorname{IG~}^{1}(1), \operatorname{IG}^{1}(1)$,

Number of batches of coefficients of different format for material 1, fol$\mathrm{IW}^{1}(\mathrm{IB}), \mathrm{IG}^{1}(\mathrm{IB}), \mathrm{IG}^{1}(\mathrm{IB})$ lowed by the batch format and batch energy-level limits.

IW $(1)=1$ Coefficicients in CMS

$I W(I)=2$ Coefficients in CMS ratio, $B_{0}$, $\mathrm{B}_{1} / \mathrm{B}_{0}, \mathrm{~B}_{2} / \mathrm{B}_{0}, \mathrm{~B}_{3} / \mathrm{B}_{0}, \mathrm{~B}_{4} / \mathrm{B}_{0}$.

$I W(I)=3$ Coefficients in LAB ratio, $A_{0}$, $A_{1} / A_{0}, A_{2} / A_{0}, A_{3} / A_{0}, A_{4} / A_{0}$.

$I W(I)=4$ Coefficients in LAB system.

IB ${ }^{\mathrm{MATl}}{ }_{\text {IW }} \mathrm{MAT}^{\mathrm{MA}}(1)$

$\mathrm{IGl}_{1} \operatorname{MAT}^{1}(1), \operatorname{IG}_{2} \operatorname{MATl}^{(1)}$,

Number of batches of coefficients of different format for material MAT $1_{\text {, }}$ followed by the batch format and batch $\left.\mathrm{IW}^{\mathrm{MAT}}{ }^{\mathrm{M}} \stackrel{\cdots}{\mathrm{IB}}\right), \mathrm{IGl}^{\mathrm{MAT}}{ }_{(\mathrm{IB})}$ energy-level limits. Each material IG $2^{\text {MATl }}$ (IB) starts a new card.

In addition, a $B C D$ tape to be mounted on logical unit 4 containing the following information must be prepared. 
$A_{0}(1,1), A_{1}(1,1), A_{2}(1,1), A_{3}(1,1), A_{4}(1,1)$

$\mathrm{A}_{0}(1, \mathrm{IGP} 1), \mathrm{A}_{1}(1, \mathrm{IGP} 1), \mathrm{A}_{2}(1, \mathrm{IGP} 1)$,

$\mathrm{A}_{3}(1, \mathrm{IGP} 1), \mathrm{A}_{4}(1, \mathrm{IGP} 1)$

$\mathrm{A}_{0}\left(\mathrm{MAT1}\right.$, IGP1), $\mathrm{A}_{1}(\mathrm{MAT1}, \mathrm{IGPl}), \mathrm{A}_{2}(\mathrm{MATl}$, IGPl), $\mathrm{A}_{3}(\mathrm{MAT1}, \mathrm{IGPl}), \mathrm{A}_{4}(\mathrm{MAT1}, \mathrm{IGPl})$
Remarks

Legendre coefficients for material 1 , energy level 1 . FORMAT (5E 12.5).

Legendre coefficients for material 1 , energy level IGPl. FORMAT (5E12.5).

Legendre coefficients for material MATl, energy level IGPl. FORMAT (5E12.5). 
VII. OUTPUT

On-line Output

The code prints out the iteration number, buckling, square root of the buckling, and $\mathrm{K}_{\mathrm{eff}}$ at each iteration, both for the ELMOE and coarsegroup fundamental-mode calculations.

Off-line

The off-line output consists of a summary of the input, initial, and final values of the buckling, and material-dependent-averaged coarse-group elastic-removal cross sections. Also, for each coarse group the flux, current/B, the diffusion coefficient, and elastic-removal cross section are listed. For each fine group the following quantities are normally written: flux, current/B (except for $\mathrm{P}_{1}$ ), slowing-down density, inelastic source, and elastic source. The complete transfer matrix can be obtained on option. For further clarification of the output, the following comments hold.

Material-dependent elastic-removal cross sections are the partial contribution from material $i$ to the homogenized-averaged coarse-group elastic-removal cross section.

Coarse-group Output

$1 / D$ - average value $3 \sigma_{t r}$ homogenized for all materials

Elastic removal - sum of elastic transfers out of the coarse group divided by the total coarse-group flux

Absorption - averaged homogenized value of $\left(\sigma_{a b s}-\sigma_{e l r}\right)$ for light elements and $\sigma_{a b s}$ for heavy elements.

Fine-group Output

Slowing-down density at $E$ is the total number of neutrons slowing down below $E$ because of elastic collisions above $E$. "A" denotes the source from higher energies due to elastic scattering. "B" denotes the source from higher energies due to inelastic scattering. "C" denotes the contribution from the current term in the "consistent" case.

Finally, a HOBO [25] equivalent is done; it is a coarse-group fundamental-mode calculation using averaged homogenized cross sections from the ELMOE calculation. The buckling obtained should be the same as for the main ELMOE calculation unless an error has occurred. The summary of macroscopic cross sections obtained has proven very useful. For the $\mathrm{P}-1$ option the information summarized in the $\mathrm{HOBO}$ equivalent output is written on the output tape with a " $Q$ " control character causing 
the information to be punched on cards. These cards may then be used (preceded by a title card) for input to an independent routine that provides a calculation of the adjoint flux.

Code Tape

The code tape itself consists of the following records, each preceded by WBTSB 2 - Absolute Binary Tape Loaders:

1. Monitor

2. Cross-section tape-reading routine

3. Legendre coefficient tape-reading routine

4. Cross-section tape-writing routine

5. Legendre coefficient tape-writing routine

6. Sodium narrow-resonance treatment

7. Scattering-matrix calculation

8. Fundamental-mode calculation

9. Output routine

Routines 7, 8, 9 are repeated for each of the dimension limits mentioned above. The code tape is written with the WB-CTB 2 - Absolute Binary Card to tape routine. To make the code tape, READY the card deck in the card reader, CLEAR, and depress LOAD CARDS. The code tape is written on logical unit $l$. 


\section{OPERATING INSTRUCTIONS}

The code requires a $32 \mathrm{~K}$ IBM 704 with 7 on-line tape units, an online printer, and an on-line card reader. The card reader uses the standard share $72 \times 72$ board, and the printer the SHARE number 2 board.

SENSE SWITCHES. All sense switches are to be in the "up" position.

Tape Units.

Tape 1 ELMOE Code

Tape 2 ELMOE Library

Tape 3 Scratch

Tape 4 Scratch, or BCD Input for Legendre coefficient writing routine.

Tape 5 Preliminary for sodium narrow resonance routine (used only for preliminary to sodium narrow resonance routine or narrow resonance routine)

Tape 7 Binary input for sodium narrow resonance routine (library supplied)

Tape 10 Output tape

$\underline{\text { Running procedure }}$

1. Mount and ready tapes

2. Underflow switch on

3. All sense switches up

4. Ready cards in reader

5. Ready printer

6. Clear, depress LOAD TAPE.

Program stops on card reader select. Write EOF on tape 10, rewind and print on Program Control. STOPS: FORTRAN program stops. 


\section{REFERENCES}

1. Weinberg, A. M., and Wigner, E. P., The Physical Theory of Neutron Chain Reactors, The University of Chicago Press, Chicago (1958).

2. Selengut, D. S., APEX-121 (1952).

3. Hurwitz, H., Jr., and Zweifel, P. F., J. Appl. Phys. 26, 923 (1955).

4. Hellens, R. L., Long, R. W., and Mount, B. H., WAPD-TM-4 (1956).

5. Hummel, H. H., and Rago, A. L., An Accurate Treatment of Resonance Scattering in Light Elements in Fast Reactors, Proc of IAEA Seminar on the Physics of Fast and Intermediate Reactors, Vienna, August 3-11, 1961. Vol. I, pp. 231-250, International Atomic Energy Agency (1962).

6. Meneghetti, D., Recent Advances and Problems in Theoretical Analyses of ZPR-III Critical Assemblies, Proc. of IAEA Seminar on the Physics of Fast and Intermediate Reactors, Vienna, August 3-11, 1961. Vol. I, pp. 457-487, International Atomic Energy Agency (1962). See also ANL- 6466.

7. Hummel, H. H., and Rago, A. L., Effect of Parameter Variation in Doppler Effect Calculations, Proc. of Conference on Breeding, Economics and Safety in Large Fast Power Reactors, October 7-10, 1963. ANL-6792.

8. Hibdon, C. T., Phys. Rev. 118, 514 (1960).

9. Hibdon, C. T., Phys. Rev. 122, 1235 (1961).

10. Hibdon, C. T., Phys. Rev. 124, 500 (1961).

11. Lane, R. O., Miller, W. F., and Hillstrom, K. E., ANL-6039 (1959).

12. Hughes, D. J., and Schwartz, R. B., BNL-325, 2nd ed. (1958), and Suppl. 1 (1960).

13. Hibdon, C. T., Phys. Rev. 114, 179 (1959).

14. Lane, R. O., and Monahan, J. E., Phys. Rev. 118, 533 (1960).

15. Langsdorf, A., Jr., Lane, R. O., and Monahan, J. E., Phys. Rev. 107, 1077 (1957). See also ANL-5567 (June 1956).

16. Yiftah, S., Okrent, D., and Moldauer, P. A., Fast Neutron Cross Sections, Pergamon Press, London (1960).

17. Machwe, M. K., et al., Phys. Rev。 114, 1563 (1959).

18. Lind, D. A., and Day, R. B., Annals of Physics, 12, 485 (1961).

19. Kiehn, R. M., and Goodman, C., Phys. Rev. 95, 989 (1954).

20. Lane, R. O., et al., Annals of Physics (Feb. 1961). 
21. Wills, J. E., et al., Phys. Rev. 109, 891 (1958).

22. Lustig, H., Goldstein, H., and Kalos, M. H., An Interim Report on the Neutron Cross Sections of Oxygen, NDA-086-2 (Jan. 31, 1958).

23. Butler, M. K., AMD Program Library RE-122.

24. Carlson, B. G., The $S_{n}$ Method and the SNG and SNK Codes, T-1-159, Los Alamos Scientific Laboratory (Jan. 1958).

25. Cavanaugh, M., AMD Program Library RE-247.

\section{ACKNOWLEDGMENT}

Extensive assistance with the computational work in preparing the library tapes was provided by K. E. Phillips, L. Kvitek, and Stella Dean. Thanks are due to C. E. Hibdon for furnishing his original data on the cross sections of aluminum and sodium. 


\section{APPENDIX}

\section{$\underline{\text { Sample Problem }}$}

The following information is given for a sample problem:

1. Input sheets

2. List of materials in cross-section set

3. List of materials in Legendre coefficient set

4. On-line output

5. Off-line output. 
704 INPUT DATA

FORM ॥

COST CODE

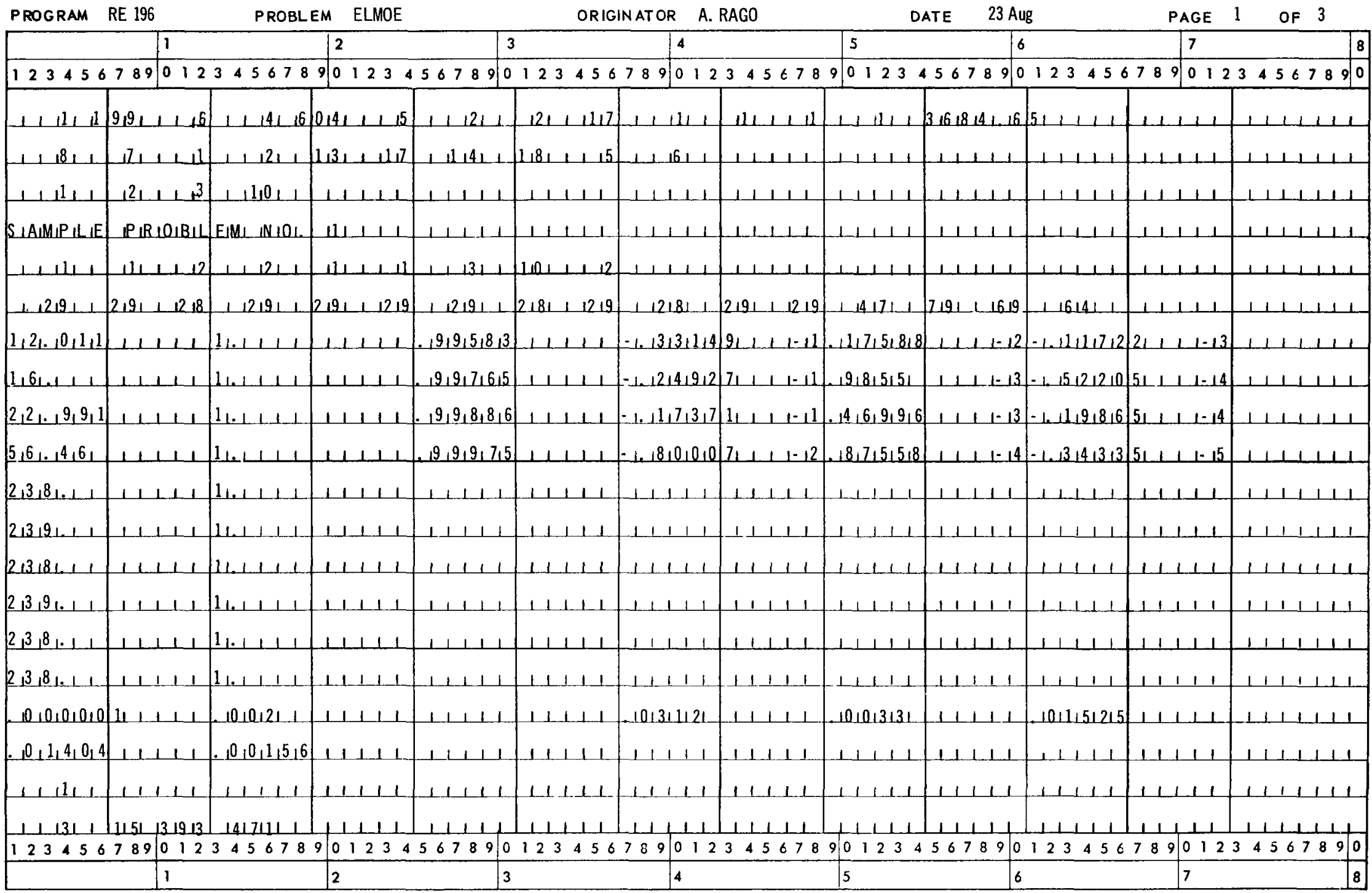

AMD.9 19.60 
704 INPUT DATA

FORM II

COST CODE

\begin{tabular}{|c|c|c|c|c|c|c|c|c|c|c|c|c|c|c|}
\hline ROGRAM & & & PROBLE & EM ELMOE & & ORIG & INATOR A. R & AG0 & & 23 Aug & & PAGE & OF & \\
\hline & & 1 & & 2 & 3 & & 4 & & 5 & & 6 & 7 & & 8 \\
\hline 123456 & 789 & $\begin{array}{lll}0 & 1 & 2\end{array}$ & 345678 & 9001123. & 15678890 & 123456 & \begin{tabular}{ll|lll}
78 & 9 & 0 & 12 \\
\end{tabular} & $345 \in 78$ & \begin{tabular}{l|llll}
9 & 0 & 1 & 2 & 3
\end{tabular} & 678 & 0123456 & \begin{tabular}{llll|l}
7 & 8 & 9 & 0 \\
\end{tabular} & 2345678 & \\
\hline 101118141 & 11 & $\perp \perp 1$. & 10111917 & $\begin{array}{lllll}1 & 1 & 1 & 1 & 1\end{array}$ & 101211121 & $\begin{array}{lllll}1 & 1 & 1 & 1 & 1\end{array}$ & .101212161 & 111 & $.10+3 \perp 11$ & $1 \cdot 1$ & $.14+111$ & 1111 & $1|1| \perp$ & \\
\hline , & 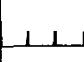 & 1.1 & $., 6,5, \ldots$ & $\begin{array}{llll}1 & 1 & 1 & 1\end{array}$ & $.1^{7}, 9,1$, & 1.1 .111 & $., 8,4, \ldots$, & & $., 9,6,1$ & 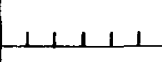 & $1, \ldots, 1,5,1$ & 1111 & 1 & \\
\hline $1_{1} \cdot 2_{1} 3_{1}$ & 11 & 111 & $1, \cdot \mu^{4}, 8, \perp$ & $\perp \perp \perp \perp L$ & $1, \cdot 1_{1}^{6}, 7_{1}$ & 111.1 .1 & $2+\cdot 4_{1} \perp \perp$ & $\perp \perp$ & $3+1^{2} \perp \perp 1$ & 1 & $44_{1} \cdot 1,1 / 1$ & $\perp \perp \perp \perp 1$ & 111 & \\
\hline $\left.2+1^{7}\right]^{8}+$ & 11 & $\perp \perp \perp$ & $1,1_{1}^{7}{ }_{1}^{8}$ & 11111 & $1, \perp_{1} \Lambda_{1}^{3} \perp \perp$ & $1 \perp 111$ & $1,1 \cdot 0_{\perp} 3_{1} L$ & 1 & $.1^{8}, 3+1 \ldots$ & & $.14_{1} \perp \perp 1$ & 11111 & 1 & \\
\hline $0,2,1,4$, & لــــــ & $1 \perp$ & $.10,1,5,9$, & لــــــــــ & $.0,1,2,7$, & 11111 & $.0_{1} 0_{1}, 9_{1} 8_{1}$ & $1 \perp \perp 1$ & $.0,0,8,3$, & 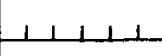 & $.0,0,7,9_{1}$ & ـــــــــــــــــ & $11+1$ & \\
\hline $1.0,0,7_{1} 1_{1}$ & LـL & 111 & $.10,0,6,4 \perp$ & 11111 & $.0,0,611$ & 11111 & $\therefore 10,0,5,61$ & 1111 & $.0_{1}, 0,5,1$, & & $.10,0,4,8$, & 11111 & 111111 & \\
\hline $1_{1}^{0}, 0,4,6$ & 1.1 & $1 \perp 1$ & $-1^{0}, 0,4,5$, & $\begin{array}{lllll}1 & 1 & 1 & 1 & 1\end{array}$ & $.1_{1}, 0,4,4$ & $1+111$ & $\left.-1^{0} \perp_{\perp} 0,4\right]_{1}$ & 11111 & $\cdot 1^{0}, 0,4,2$, & 11111 & $.10,0,4,1$, & 11111 & $1|1| 1|1| 1$ & \\
\hline$\perp_{1}^{0}{ }_{1}^{0}, 3,{ }_{1}$ & 11 & 111 & . $\left.0_{1}^{0} 0_{1}^{0}\right]_{1}^{8}$ & 11111 & $.{ }_{1}, 0,0,3,7$ & $\begin{array}{lllll}1 & 1 & 1 & 1 & 1\end{array}$ & $\cdot 1_{1}^{0}, 0,3,6$, & 1.1111 & - $1^{0}, 0,3,1_{1}$ & $11 \perp 11$ & $\cdot 0,0,3,4$ & $1 \perp \perp 1$ & & \\
\hline $1_{1}{ }_{1} 0,1_{1}^{3}{ }_{1}$ & 1 & 111 & - $1_{1}^{0} 1_{1}^{3} 1_{1}^{2}$ & 11111 & $\cdot 1^{0}, 0,3,1$ & $1 \begin{array}{lllll}1 & 1 & 1 & 1 & 1\end{array}$ & $\cdot 1_{1}, 0,3,0$, & 11111 & $., 0,0,2,9,5$ & $1 \perp \perp \perp 1$ & $.10,0,2,9$ & $1 \perp 1 \ldots$ & 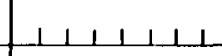 & \\
\hline $10,0,2,8,5$ & | & $1 \perp$ & $.10,0,2,8$ & $1 \perp \perp 11$ & $., 0,0,2,7,8$ & 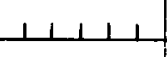 & . ${ }^{0}, 0,2,7,6$ & & $-10,0,2,7,4$ & & $=10,0,2,7,2$ & & 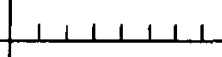 & \\
\hline$=0,0,2,7$, & 11 & 111 & $., 0,0,2,6,8$ & 11111 & $.0,0,2,6,6$ & 11111 & $.0,0,2,6,4$ & 11111 & $\mid 0,0,2,6,2$ & $1|1|$ & $.0,0,2,6,0$ & 11111 & 1111,11 & \\
\hline$-10,0,2,5,8$ & 11 & 1.1 .1$. & $.10,0,2,5,6$ & 11111 & $\cdot \perp^{0}, 0,2,5,4$ & 111111 & $\therefore, 10,0,2,5,2$ & 11111 & $=1_{1}^{0}, 0,2,5,0$ & 1111.11 & $\therefore 0,0,2,4,8$ & 111111 & $\perp 1111,111$ & \\
\hline $.0,0,2,4,7$ & 11 & 111 & $., 0,0,2,4,7$ & 11111 & $\cdot 1^{0}, 0,2,4,7$ & $\begin{array}{lllll}1 & 1 & 1 & 1 & 1\end{array}$ & $.0,0,2,4,7$ & 111111 & $1^{0}, 0,2,4,7$ & 11111 & $\cdot 1,0,0,2,4,7$ & 111111 & $1|1| 1|1|$ & \\
\hline $0,0,2,4,7$ & 11 & 1.1 & $1 \perp 111$ & $\begin{array}{lllll}1 & 1 & 1 & 1 & 1\end{array}$ & $\perp \perp \perp \perp 1$ & 11111 & $\begin{array}{lllll}1 & 1 & 1 & 1 & 1\end{array}$ & 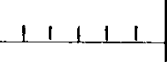 & & 1.1111 & & 111111 & $1 \perp 1 \perp 111$ & \\
\hline$S_{1} A_{1} M_{1} P_{1} L_{1} E$ & $P_{\perp} P_{\perp}$ & $\mathrm{O}_{1} \mathrm{~B}, \mathrm{~L}$ & $E_{1} M_{\perp} \perp N_{1} O_{ل}$. & $|2| I_{1}|1|$ & $\mid 1 \perp 1 \perp 1$ & 1111.11 & $\begin{array}{lllll}1 & 1 & 1 & 1 & 1\end{array}$ & $1: 111$ & $\mid \begin{array}{lllll}1 & 1 & 1 & 1 & 1\end{array}$ & $\mid \perp \perp \perp \perp$ & $\mid \begin{array}{lllll}1 & 1 & 1 & 1 & 1\end{array}$ & $\mid \begin{array}{lllll}1 & 1 & 1 & 1 & 1\end{array}$ & $\mid \begin{array}{llllll}1 & 1 & 1 & 1 & 1 & 1\end{array}$ & \\
\hline $.0,0,0,0,0$ & $1 \pm 1$ & 111 & $.10,0,2,1$ & 11111 & $\left|\begin{array}{lllll}1 & 1 & 1 & 1 & 1\end{array}\right|$ & 11111 & $.103_{1} 1_{1} 22_{1}$ & $\perp 1111$ & $=0,0,3,3$, & 11111 & $=10,1,5,2,5$ & 11111 & 11111111 & \\
\hline 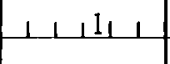 & 11 & 111 & $\mid \begin{array}{lllll}1 & 1 & 1 & 1 & 1\end{array}$ & 11111 & $.10,1,4,0,4$ & 11111 & $.10,0,1,5,6$ & 11111 & $11+11$ & $1 \perp 1 \perp \perp$ & $-\begin{array}{lllll}1 & 1 & 1 & 1 & 1\end{array}$ & 111111 & $\mid \begin{array}{llllll}1 & 1 & 1 & 1 & 1 & 1\end{array}$ & \\
\hline $1,1_{1}^{3} 1$ & 1,5, & {$\left[3,1^{9}, 3\right.$} & $14,7,1,1$ & 11111 & $\downarrow\llcorner\perp \perp 1$ & 11111 & 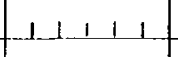 & 11111 & $\left|\begin{array}{lllll}1 & 1 & 1 & 1 & 1\end{array}\right|$ & 11111 & $\begin{array}{lllll}1 & 1 & 1 & 1 & 1\end{array}$ & $11+11$ & 111111.111 & \\
\hline $.10,1,8,41$ & 11 & 111 & $.1011,9171$ & $\begin{array}{lllll}1 & 1 & 1 & 1 & 1\end{array}$ & $.10,2,1,21$ & $1 ـ 1$ & $.1012,2,6]$ & 11111 & $.10,3,1,1$ & 11111 & $|4| 1,1 \ldots 1$ & $1,1 \ldots$ & $111 / 111$ & \\
\hline $151|1| 1 \mid$ & $\mid 11$ & 11 & $16151<1 \mid$ & 41111 & 1.171211 & $1 \perp \perp \perp 1$ & 1.10144 & $\perp \perp \perp \perp \perp$ & $L Y Y 10 L \perp \perp A$ & $1111+$ & 10.16] & لـ & $1+1,1111+$ & \\
\hline 23456 & & & 345678 & $9 \begin{array}{llllll}9 & 0 & 1 & 2 & 3 & 4 \\
\end{array}$ & 90 & 123456 & $\begin{array}{ll}789 & 0 \\
\end{array}$ & 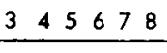 & 90101234 & $\begin{array}{llllll}5 & 6 & 7 & 8 & 9 & 0\end{array}$ & $\begin{array}{llllll}123 & 3 & 5 & 6\end{array}$ & \begin{tabular}{ll|lll}
7 & 8 & 9 & 0 & 1
\end{tabular} & 34567890 & \\
\hline & & 1 & & 2 & 3 & & & & 5 & 6 & & 7 & & \\
\hline
\end{tabular}


704 INPUT DATA

FORM ॥

COST CODE

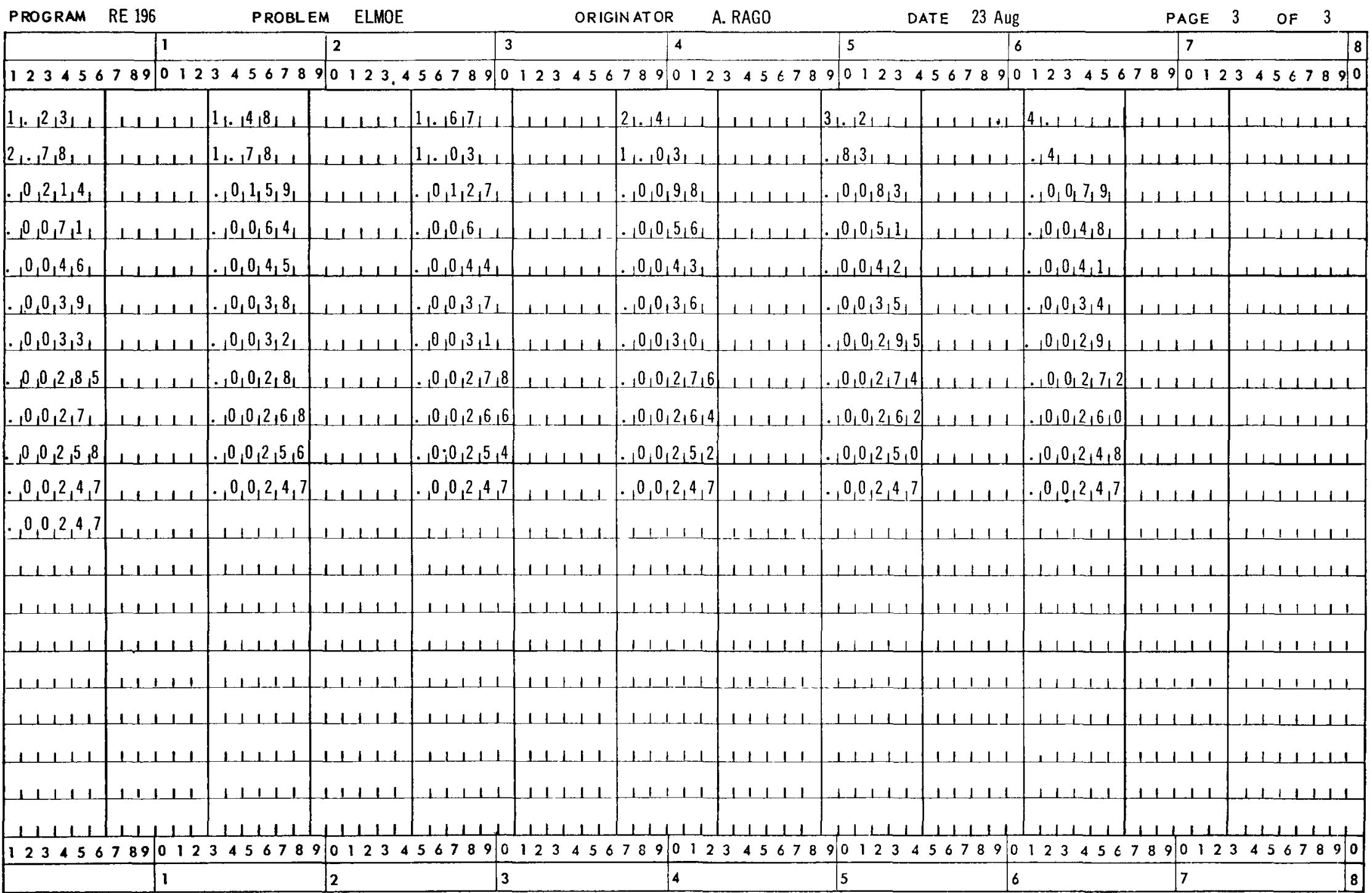


CROSS-SECTION SET \#199

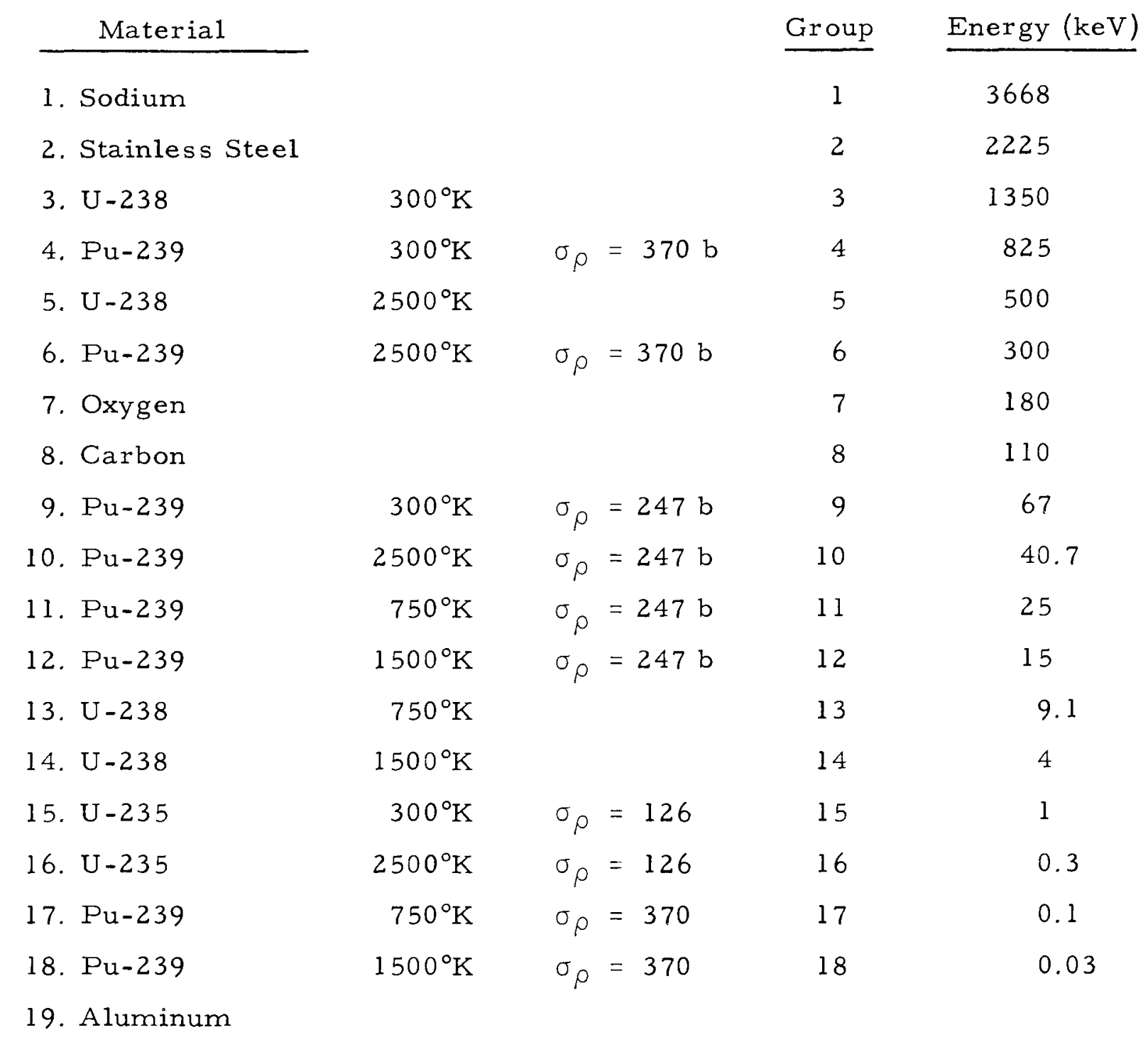

\section{LEGENDRE COEFFICIENT SET}

\section{Material}

1. Carbon

2. Oxygen

3. Sodium

4. Sodium- 1

5. Sodium-2

6. Aluminum

7. Vanadium
Atomic Weight

12.011

16.000

22.991

23.000

23.010

26.980

50.950
Material

8. Chromium

9. Iron

10. Stainless Steel

11. Hastelloy

12. Nickel

13. Niobium
Atomic Weight

52.010

55.840

56.460

56.500

58.690

92.910 


\section{ON-LINE OUTPUT}

RE 196

ELMOE

L E P R O B L E M N O . 1

IT. NO. 1 B SQUARE $=0.00200000$

IT. NO. 2 B SQUARE $=0.00241999$

$B=0.04472136$

SUM $=$

1.03785206

IT. NO. 3 B SQUARE $=0.00240569$

$B=0.04919349$

SUM $=0.99872560$

IT. NO. 4

B SQUARE $=0.00240587$

$B=0.04904783$

SUM $=$

1.00001706

$B=0.04904975$

SUM $=$

0.99999997

HOBO EQUIVALENT

IT. NO.

B SCUARE $=0.00200000$

$B=0.04472136$

SUM $=1.03794806$

IT. NO.

B SQUARE $=0.00241999$

$B=0.04919349$

SUM $=0.99872561$

IT. NO. 3

B SQUARE $=0.00240572$

$B=0.04904819$

SUM $=$

1.00001651

IT. NO. 4

B SQUARE $=0.00240590$

$B=0.04905004$

SUM $=1.00000000$

RE 196 ELMOE

SAM P LE PR D B LEM N D . 2

IT. NO. 1 B SQUARE $=0.00200000$

$B=0.044 / 2136 \quad$ SUM $=1.03354345$

IT. NO.

B SQUARE $=0.00241999$

$B=0.04919349$

SUM $=0.99482176$

IT. NO. 3

8 SQUARE $=0.00236151$

$B=0.04859544$

SUM $=$

1.00006440

IT. NO. 4

B SQUARE $=0.00236223$

$B=0.04860278$

SUM $=1.00000012$

MOBO EQUIVALENT

IT. NO.

B SQUARE $=0.00200000$

$B=0.04472136$

SUM

1.03364480

IT. NO. 2

B SQUARE $=0.00241999$

$B=0.04919349$

SUM $=0.99481191$

IT. NO. 3

B SQUARE $=0.00236157$

$B=0.04859601$

SUM $=1.00006203$

IT. NO.

B SQUARE $=0.00236226$

$B=0.04860307$

SUM $=1.00000010$ 


\title{
OFF-LINE OUTPUT
}

RE 196

P L E P P R D B LE M NO. 1

$\begin{array}{ccr}\text { MATERIAL } & \text { AT.WT } & \text { ATOMS/CCIE-24 } \\ 1 & 12.011000 & 1.00000000 \\ 2 & 16.000000 & 1.00000000 \\ 3 & 22.991000 & 1.00000000 \\ 4 & 56.460000 & 1.00000000 \\ 5 & 238.000000 & 1.00000000 \\ 6 & 239.000000 & 1.00000000 \\ 7 & 238.000000 & 1.00000000 \\ 8 & 239.000000 & 1.00000000 \\ 9 & 238.000000 & 1.00000000 \\ 10 & 239.000000 & 1.00000000\end{array}$

MATERIAL CONSTANTS

MATRIX FOR TRANSFORMING LEGENDRE EXPS. COEFF.-CMS TO LAB

$$
(1,1)
$$

$$
(1,2)
$$

$(1,3)$

$$
(1,4)
$$

$0.99583 E \quad 00 \quad-0.33149 E-01$

$0.99765 E 00-0.24927 E-01$

$0.99886 \mathrm{E} 00 \quad-0.17371 \mathrm{E}-01$

$0.99975 E \quad 00 \quad-0.80007 E-02$
$0.17588 \mathrm{E}-02$ $0.98550 \mathrm{E}-03$

$0.46996 \mathrm{E}-03$

$0.46996 \mathrm{E}-03$
$0.87558 \mathrm{E}-04$
$-0.11722 E-03$

$-0.52205 E-04$

$-0.19865 E-04$

$-0.34335 E-05$

$\mathrm{R}=$

\begin{abstract}
0.98274157
\end{abstract}
OPTION 1

$\begin{array}{cc}\text { CALCULATED } \\ \text { MATER IAL } \\ \text { ALPHA } \\ 1 & 0.2816316 E-00 \\ 2 & 0.2162987 E-00 \\ 3 & 0.1597795 \mathrm{E}-00 \\ 4 & 0.6726708 \mathrm{E}-01\end{array}$

0.01403999 0.00156000

$-0$.

$-0$.

$-0$ 
MATERIAL DEPENDENT ELASTIC REMOVAL CROSS SECTION

GROUP

$\begin{array}{ll}2 & -0 . \\ 3 & -0 . \\ 4 & -0 . \\ 5 & -0 . \\ 6 & -0 . \\ 7 & -0 . \\ 8 & -0 . \\ 9 & -0 . \\ 10 & -0 . \\ 11 & -0 . \\ 12 & -0 . \\ 13 & -0 . \\ 14 & -0 . \\ 15 & -0 . \\ 16 & -0 . \\ 17 & -0 .\end{array}$

MATERIAL

c. $7827 \mathrm{E}-02 \quad 0.1056 \mathrm{E}-02 \quad 0.1500 \mathrm{E}-02$ $0.1759 \mathrm{E}-010.9204 \mathrm{E}-03 \quad \mathrm{C} 0.1558 \mathrm{E}-02$ $0.3532 \mathrm{E}-010.2038 \mathrm{E}-020.3404 \mathrm{E}-02$ $0.2114 \mathrm{E}-01 \quad 0.1651 \mathrm{E}-02 \quad 0.2704 \mathrm{E}-02$ $\begin{array}{lll}0.4344 \mathrm{E}-01 & 0.2417 \mathrm{E}-02 & 0.2512 \mathrm{E}-02\end{array}$ $0.2775 \mathrm{E}-01 \quad 0.2568 \mathrm{E}-02 \quad 0.3431 \mathrm{E}-02$ $0.2521 \mathrm{E}-010.1766 \mathrm{E}-02 \quad 0.3555 \mathrm{E}-02$ $0.2495 \mathrm{E}-010.2141 \mathrm{E}-02 \quad 0.3462 \mathrm{E}-02$ $0.2491 \mathrm{E}-01 \quad 0.2 \mathrm{C} 22 \mathrm{E}-02 \quad 0.4772 \mathrm{E}-02$ $0.2782 \mathrm{E}-01 \quad 0.2907 \mathrm{E}-02 \quad 0.4895 \mathrm{E}-02$ $0.2020 E-01 \quad 0.1896 E-02 \quad 0.8291 E-02$ $0.2263 E-01 \quad 0.2744 E-02 \quad 0.6788 E-02$ $0.1381 \mathrm{E}-01 \quad 0.4840 \mathrm{E}-02 \quad \mathrm{C} .5664 \mathrm{E}-02$ $0.6703 E-02 \quad 0.4087 E-03 \quad 0.2311 E-02$ $0.5482 E-02 \quad 0.3335 E-03 \quad 0.2241 E-02$ $0.4009 E-02 \quad 0.2410 E-03 \quad 0.1647 E-02$

BUCKLING

INITIAL VALUE $0.2000000 E-02$ FINAL VALUE $0.2405878 E-02$ COARSE GROUP DATA

FINE GROUP

$\begin{array}{ccccc}\text { GROUP } & \text { CORRESPONDENCE } & \text { FLUX } & \text { CURRENT/B } \\ 1 & 0-0 & 0.147416 E \text { O } 1 & 0 . \\ 2 & 1-29 & 0.299984 \mathrm{E} 01 & 0 . \\ 3 & 30-58 & 0.433799 \mathrm{E} 01 & 0 . \\ 4 & 59-86 & 0.564262 \mathrm{E} 01 & 0 . \\ 5 & 87-115 & 0.123874 \mathrm{E} 02 & 0 . \\ 6 & 116-144 & 0.894581 \mathrm{E} 01 & 0 . \\ 7 & 145-173 & 0.115149 \mathrm{E} 02 & 0 . \\ 8 & 174-202 & 0.111647 \mathrm{E} 02 & 0 . \\ 9 & 203-230 & 0.859068 \mathrm{E} 01 & 0 . \\ 10 & 231-259 & 0.731513 \mathrm{E} 01 & 0 . \\ 11 & 260-287 & 0.512522 \mathrm{E} 01 & 0 . \\ 12 & 288-316 & 0.428785 \mathrm{E} 01 & 0 . \\ 13 & 317-345 & 0.285616 \mathrm{E} 01 & 0 . \\ 14 & 346-392 & 0.243306 \mathrm{E} 01 & 0 . \\ 15 & 393-471 & 0.183030 \mathrm{E} 01 & 0 . \\ 16 & 472-540 & 0.475049 \mathrm{E}-00 & 0 . \\ 17 & 541-604 & 0.631634 \mathrm{E}-01 & 0 . \\ 18 & 0- & 0 & 0.558255 \mathrm{E}-02 & 0 .\end{array}$

ELASTIC REMOVAL

$0.39255 E-00$ $0.43178 E-00$ $0.50863 \mathrm{E} \quad 00$ $0.70405 E 00$ $0.64267 \mathrm{E} 00$ $0.97859 \mathrm{E} 00$ $0.94079 E \quad 00$ $0.10140 E$ OI $0.11142 E 01$ $0.11639 E$ O 1 $0.13713 E \quad 01$ $0.12465 E 01$ 0.14256 E 01 $0.17734 \mathrm{E}$ OI 0.16978 E 01 0.17987 E 01 $0.20865 \mathrm{E} 01$ $0.40654 \mathrm{E} \quad 01$
$0.10383 E-01$ $0.20070 \mathrm{E}-01$ $0.20070 \mathrm{E}-01$ $0.25496 E-0$ $0.48365 E-01$ $0.33751 E-01$ $0.3 \cup 528 E-01$ $0.30551 \mathrm{E}-0$ $0.31708 E-01$ $0.35618 \mathrm{E}-01$ $0.30383 E-01$ $0.32166 \mathrm{E}-01$ $0.24311 E-01$ $0.94224 \mathrm{E}-02$ $0.80564 E-02$ $0.58966 \mathrm{E}-02$ $0.64480 E-01$ $0.48890 \mathrm{E}-01$ $0.24633 E-01$ $0.13707 E-01$

$0.12802 \mathrm{E}-01$ $0.14007 \mathrm{E}-01$ $0.14613 E-01$ $0.15775 E-01$ $0.13817 E-01$ $0.12710 \mathrm{E}-01$ $0.16420 \mathrm{E}-01$ $0.16621 \mathrm{E}-01$ $0.15216 \mathrm{E}-01$ $0.25014 \mathrm{E}-\mathrm{Cl}$ $0.36893 \mathrm{E}-01$ 
GROUP ENERGY

FLUX

CURRENT/B

$0.381869 \mathrm{~F}-\mathrm{Cl}$ $0.461638 \mathrm{E}-01$ $0.5120 C 4 E-C 1$ $0.551196 \mathrm{E}-01$ C. $605298 \mathrm{E}-01$ $0.630217 E-01$ $0.652949 \mathrm{E}-01$ $0.727598 \mathrm{E}-01$ $0.836085 \mathrm{E}-\mathrm{Cl}$ $0.927708 E-01$ $0.997940 \mathrm{E}-01$ $0.104242 E-00$ $0.108212 \mathrm{E}-00$ $0.113019 E-00$ $0.118208 E-00$ $0.120698 E-00$ $0.121731 t-C O$ $0.123555 \mathrm{E}-\mathrm{CO}$ $0.124180 \mathrm{E}-00$ $0.124180 \mathrm{E}-00$ $0.124003 E-G 0$ $0.123661 t-C 0$ $0.123183 \mathrm{E}-\mathrm{CO}$ $0.125760 t-00$ $0.132864 E-00$ $0.139190 \mathrm{E}-\mathrm{CO}$ $0.158060 E-C O$ $0.147534 \mathrm{E}-00$ C. $134008 \mathrm{E}-\mathrm{CO}$ D. 12928 CE-OO $0.152123 E-C O$ $0.153985 \mathrm{E}-00$ C. 1509 CCE-0O $0.150297 \mathrm{E}-\mathrm{CO}$ $0.148434 E-00$ $0.150083 \mathrm{E}-00$ $0.150083 \mathrm{E}-00$ $0.146062 \mathrm{E}-\mathrm{CO}$ $0.137442 E-00$ $0.131472 \mathrm{E}-00$ $0.132607 \mathrm{E}-00$ $0.141134 \mathrm{E}-00$ $0.153679 E-00$ $0.166355 \mathrm{E}-00$ $0.166429 \mathrm{E}-00$ $0.166455 \mathrm{E}-00$ $0.156929 \mathrm{E}-00$ $0.134142 \mathrm{E}-00$ $0.135357 \mathrm{E}-00$ $0.149669 \mathrm{E}-00$ $0.154555 \mathrm{E}-00$ $0.147203 \mathrm{E}-00$ $0.149151 \mathrm{E}-00$ $0.154201 E-00$
35IGMA

SLCWING CCAN

DENSITY

$0.578167 \mathrm{E}$ OO $0.451121 \mathrm{E}-02 \quad 0$

$0.579073 \mathrm{E}$ CO $0.858492 \mathrm{~F}-32 \quad 0.12611 \mathrm{E}-02$ 0.58358 $0.585475 E$ DO $0.160978 E-01$ $0.565383 \mathrm{E}$ 00 $0.19497 \mathrm{CE}-01$ $0.560325 E$ CO C.228237E-0I 0.5629875 กO $0.524412 E$ O0 $0.475361 t-00$ C. $441149 \mathrm{E}-\mathrm{CO}$ $0.423608 t-0 C$ $0.417609 t-00$ 0.417609 $0.417736 E-00$ $0.418482 \mathrm{E}-00$ $0.418607 \mathrm{E}-00$ $0.418965 E-C O$ $0.423487 E-00$ $0.423092 t-00$ $0.423092 t-60$ $0.425408 E-00$
$0.429421 E-00$ $0.429421 E-00$
$0.434596 E-00$ $0.441315 t-00$ $0.429546 \mathrm{E}-0 \mathrm{O}$ $0.411907 \mathrm{C}$

$\vec{i}-\mathrm{CO}$

$0.390903 t-00$ $0.358699 E-00$ $0.366410 \mathrm{t}-00$ $0.406397 t-00$ $0.421828 E-00$ 0.42182 0.4275435 $0.436533 E-00$ $0.448328 \mathrm{E}-00$ $0.460356 E-00$ $0.464506 E-00$ $0.467432 t-00$ $0.467432 t-00$ $0.476897 \mathrm{E}-00$

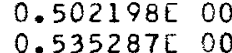
$0.539848 t 00$ $0.523965 \mathrm{E} \quad 00$ $0.497812 \mathrm{E}-0$ $0.469285 \mathrm{E}-00$ $0.472146 \mathrm{E}-00$ $0.482025 t-00$ $0.500742 \mathrm{E} \quad 00$ $0.599282 \mathrm{E} \mathrm{DO}$ $0.624003 t \quad 00$ $0.552703 E 00$ $0.534671 \mathrm{E} 00$ $0.547726 \mathrm{E} \mathrm{OO}$ $0.558200 \mathrm{E}$ OO $0.544152 \mathrm{E} \quad 00$ $0.20458 E-02$ $0.26601 E-02$ $0.31473 E-02$ C. $35397 \mathrm{E}-02$ $0.39142 \mathrm{E}-02$ $0.42738 E-02$ $0.46322 \mathrm{E}-02$ $0.49186 \mathrm{E}-02$ $0.52011 E-02$ $0.54857 E-02$ $0.58746 E-02$ $0.64093 \mathrm{E}-02$ C. $69481 \mathrm{E}-02$ $0.7204 \mathrm{CE}-02$ C. $74600 E-02$ C. $75738 \mathrm{E}-02$ $0.76450 E-02$ $0.77281 \mathrm{E}-02$ C. $77565 \mathrm{E}-02$ $0.77529 E-02$ C. $75990 E-02$ $0.76691 \mathrm{E}-02$ $0.76172 \mathrm{E}-02$ $0.77915 E-02$ $0.74457 \mathrm{E}-02$ $0.74926 \mathrm{E}-02$ $0.74044 E-02$ $0.70491 \mathrm{E}-02$ $0.76554 \mathrm{E}-02$ $0.79491 \mathrm{E}-02$ $0.82683 \mathrm{E}-02$ $0.83817 E-02$ $0.86055 \mathrm{E}-02$ $0.86592 \mathrm{E}-02$ $0.87629 \mathrm{E}-02$ $0.92250 \mathrm{E}-02$ $0.95327 \mathrm{E}-02$ $0.10078 \mathrm{E}-01$ C. $10574 E-01$ 0. $10879 \mathrm{E}-01$ $0.11225 \mathrm{E}-01$ $0.11601 E-01$ $0.11718 \mathrm{E}-01$ $0.12291 E-01$ $0.12706 E-01$ $0.12196 \mathrm{E}-01$ $0.12156 \mathrm{E}-01$ $0.12033 E-01$ $0.12593 E-01$ $0.12987 \mathrm{E}-01$
$0.12317 E-02 \quad 0$ $0.12105 \mathrm{E}-02 \quad 0$. $0.11896 E-02 \quad 0$. $0.11690 \mathrm{E}-02 \quad 0$. $0.11489 \mathrm{E}-02 \quad 0$. $0.11290 \mathrm{E}-02 \quad 0$ $0.1 C 904 E-02 \quad 0$. $0.10716 \mathrm{E}-02 \quad 0$. $0.10531 \mathrm{E}-02 \quad 0$. $0.10349 \mathrm{E}-02 \quad 0$. $0.10171 E-02 \quad 0$. $0.99950 E-03 \quad 0$. $0.98225 \mathrm{E}-03 \quad 0$. $0.96530 E-03 \quad 0$. $0.94864 E-03 \quad 0$. $0.93227 E-03 \quad 0$. $0.91618 \mathrm{E}-03 \quad 0$. $0.90037 E-03 \quad 0$. $0.88483 E-03 \quad 0$. $0.86956 \mathrm{E}-03 \quad 0$. $0.85455 \mathrm{E}-03 \quad 0$. $0.83980 \mathrm{E}-03 \quad 0$. $0.82531 \mathrm{E}-03 \quad 0$. $0.81107 \mathrm{E}-03 \quad 0$. $0.79707 \mathrm{E}-03 \quad 0$. $0.78331 \mathrm{E}-03$ 0. $0.76979 \mathrm{E}-03$ 0. $0.75651 E-03 \quad 0$. $0.24606 \mathrm{E}-02 \quad 0$. $0.24181 \mathrm{E}-02 \quad 0$. $0.23764 E-02 \quad 0$. $0.23353 \mathrm{E}-02$ 0 . $0.22950 E-02$. $0.22165 E-02 \quad 0$. $0.21783 E-02 \quad 0$. $0.21407 \mathrm{E}-02 \quad 0$. $0.21037 \mathrm{E}-02 \quad 0$. $0.20674 \mathrm{E}-02 \quad 0$. $0.20317 E-02 \quad 0$. $0.19967 E-02 \quad 0$. $0.19622 \mathrm{E}-02 \quad 0$. $0.19283 E-02 \quad 0$. $0.18951 \mathrm{E}-02$ 0. $0.18624 \mathrm{E}-02 \quad 0$. $0.18302 \mathrm{E}-02 \quad 0$. $0.17986 \mathrm{E}-02$ O. $0.17676 \mathrm{E}-02 \quad 0$. $0.17371 E-02 \quad 0$. $0.17071 \mathrm{E}-02 \quad 0$. 0.16776 E-02 0 . 


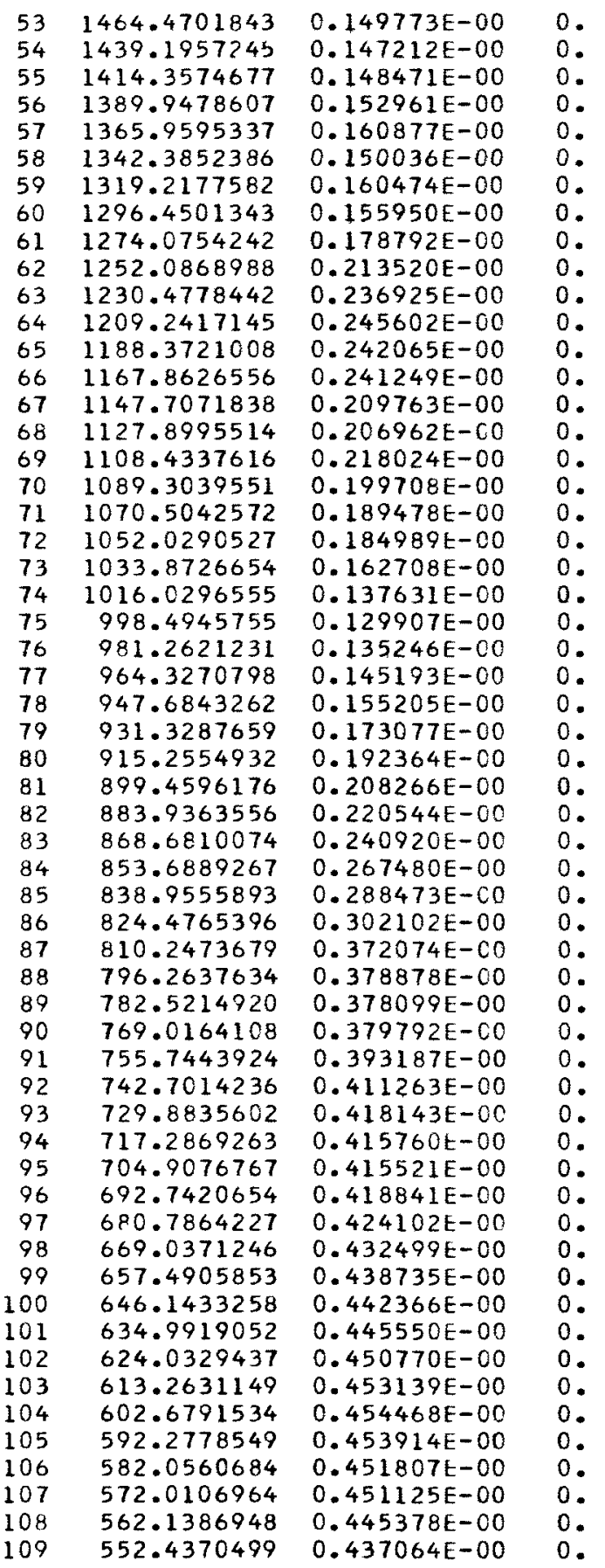

$0.550596 E$ OO $0.817735 E-01$ $0.573963 \mathrm{t} 00$ $0.571312 \mathrm{E} \quad 00$ $0.548014 \mathrm{E} \quad 00$ $0.514728 \mathrm{E} 00$ $0.497405 E-00$ $0.592429 E \quad 00$ $0.733206 \mathrm{E} \mathrm{OO}$ 0.739039 E OO $0.677383 \mathrm{E} \quad 00$ $0.643746 E$ EO $0.622764 E \quad 00$ $0.611033 E$ CO $0.617106 \mathrm{E}$ 0O $0.683196 E$ OO $0.688828 \mathrm{E} 00$ $0.653334 \mathrm{t}$ OO $0.696769 E \quad 00$ 0.729429 t CO $0.760902 t$ OO $0.881805 \mathrm{E} 00$ $0.104099 E$ DI $0.107594 \mathrm{E} \quad 01$ $0.102161 \mathrm{E} 01$ 0.972137 E 00 $0.914432 t \quad 00$ $0.822282 \mathrm{E} \quad 00$ $0.739510 \mathrm{E}$ OO $0.702524 \mathrm{E} 30$ $0.678386 E$ OO 0.652287 t 00 $0.652287 \mathrm{E} 00$ $0.642509 \mathrm{E}$
$0.639438 \mathrm{E} 00$ $0.635051 E$ OD $0.647413 E \quad 00$ $0.653574 E \quad 00$ $0.667403 E \quad 00$ $0.679891 \mathrm{E} 30$ 0.679220 t 00 0.661661 E 00 0.649880 E CC $0.653434 E \quad 00$ $0.658563 \mathrm{E}$ OO 0.659002 t 00 0.657840 E 00 0.653190 E OO $0.646517 E$ OC 0.640590 E OC $0.635620 \mathrm{t} \quad 00$ $0.631819 E \quad 00$ $0.629593 \mathrm{E} \quad 00$ 0.630407 t 00 $0.633423 E \quad 00$ 0.637090 E 00 $0.635485 E \quad 00$ $0.632586 t$ oC $0.632623 t \quad 00$
C. $13152 \mathrm{E}-01 \quad 0.16487 \mathrm{E}-02$ $0.13947 \mathrm{E}-01 \quad 0.16202 \mathrm{E}-02 \quad 0$. $0.14258 E-010.15923 E-020$. $0.14745 \mathrm{E}-01 \quad 0.15648 \mathrm{~F}-02 \quad 0$. $0.15709 \mathrm{E}-01 \quad 0.15378 \mathrm{E}-02 \quad 0$. $0.16695 \mathrm{E}-01 \quad 0.15112 \mathrm{E}-02 \quad 0$. $0.18117 \mathrm{E}-01 \quad 0.54662 \mathrm{E}-020$. $0.21439 E-01 \quad 0.53719 E-02$ $0.22000 E-010.52792 E-020$ $0.22051 \mathrm{E}-01 \quad 0.51881 \mathrm{E}-02$ $0.22633 E-01 \quad 0.50995 E-02$ $0.22562 \mathrm{E}-010.50106 \mathrm{E}-02 \quad 0$. $0.21900 E-01 \quad 0.49241 E-n 2 \quad 0$. $0.22535 \mathrm{E}-010.44391 \mathrm{E}-02$ 0. $0.22690 E-01 \quad 0.47556 t-02 \quad 0$ $0.22331 E-01 \quad 0.46735 E-02$ $0.22467 E-01 \quad 0.45929 E-02$ $0.22626 \mathrm{E}-01 \quad 0.45136 \mathrm{E}-02 \quad 0$. C. $23050 E-01 \quad 0.44357 t-02 \quad 0$. $0.24239 \mathrm{E}-010.43591 \mathrm{E}-020$ $0.26643 E-01 \quad 0.42839 E-02$ $0.28032 \mathrm{E}-01 \quad 0.421 \cap 0 \mathrm{E}-02$ C. $28380 E-01 \quad 0.41373 E-02$ C. $28363 \mathrm{E}-0 \mathrm{I} \quad 0.40659 \mathrm{E}-02$ C. $28175 \mathrm{E}-01 \quad 0.39957 \mathrm{E}-02$ C. $27360 E-01 \quad 0.39268 E-02$ $0.26940 E-01$ C. $27040 E-01$ $0.28186 \mathrm{E}-01$ $0.28974 \mathrm{E}-01$ $0.31136 \mathrm{~F}-01$ $0.34822 E-01$ $0.38244 E-01$ $0.40431 E-01$ $0.43336 E-01$ C. $45916 \mathrm{E}-01$ $0.47687 E$ $0.49752 \mathrm{E}-01$ C. $5165 \mathrm{CE}-01$ C. $52534 \mathrm{E}-01$ C. $52898 \mathrm{E}-01$ $0.53673 E-01$ $0.54602 \mathrm{E}-01$ $0.55373 E-01$ $0.55992 \mathrm{E}-01$ $0.56705 E-01$ $0.56871 \mathrm{E}-01$ $0.56984 \mathrm{E}-01$ $0.57260 E-01$ $0.58023 E-01$ $0.59005 E-01$ $0.60288 E-01$ $0.61441 \mathrm{E}-01$ C. $62404 \mathrm{E}-01$ $0.63123 \mathrm{E}-01$ $0.63384 \mathrm{E}-01$ $0.63973 E-01$ 


$\begin{array}{llll}110 & 542.9028625 & 0.432950 \mathrm{E}-00 & 0 . \\ 111 & 533.5332031 & 0.433490 \mathrm{E}-00 & 0 . \\ 112 & 524.3252563 & 0.439089 \mathrm{E}-00 & 0 . \\ 113 & 515.2762222 & 0.442510 \mathrm{E}-00 & 0 . \\ 114 & 506.3833733 & 0.440906 \mathrm{E}-00 & 0 . \\ 115 & 497.6439857 & 0.435982 \mathrm{E}-00 & 0 . \\ 116 & 489.0554314 & 0.440543 \mathrm{E}-00 & 0 . \\ 117 & 480.6151085 & 0.411922 \mathrm{E}-00 & 0 . \\ 118 & 472.3204422 & 0.357644 \mathrm{E}-00 & 0 . \\ 119 & 464.1689262 & 0.297682 \mathrm{E}-00 & 0 . \\ 120 & 456.1581039 & 0.243552 \mathrm{E}-00 & 0 . \\ 121 & 448.2855225 & 0.199000 \mathrm{E}-00 & 0 . \\ 122 & 440.5488243 & 0.181351 \mathrm{E}-00 & 0 . \\ 123 & 432.9456444 & 0.174832 \mathrm{E}-00 & 0 . \\ 124 & 425.4736786 & 0.180299 \mathrm{E}-00 & 0 . \\ 125 & 418.1306686 & 0.203610 \mathrm{E}-00 & 0 . \\ 126 & 410.9143906 & 0.223810 \mathrm{E}-00 & 0 . \\ 127 & 403.8226547 & 0.229823 \mathrm{E}-00 & 0 . \\ 128 & 396.8533058 & 0.242169 \mathrm{E}-00 & 0 . \\ 129 & 390.0042381 & 0.251917 \mathrm{E}-00 & 0 . \\ 130 & 383.2733803 & 0.265768 \mathrm{E}-00 & 0 . \\ 131 & 376.6586838 & 0.290353 \mathrm{E}-00 & 0 . \\ 132 & 370.1581421 & 0.316016 \mathrm{E}-00 & 0 . \\ 133 & 363.7697945 & 0.314636 \mathrm{E}-00 & 0 . \\ 134 & 357.4916992 & 0.328296 \mathrm{E}-00 & 0 . \\ 135 & 351.3219528 & 0.362918 \mathrm{E}-00 & 0 . \\ 136 & 345.2586861 & 0.343667 \mathrm{E}-00 & 0 . \\ 137 & 339.3000641 & 0.341439 \mathrm{E}-00 & 0 . \\ 138 & 333.4442787 & 0.357929 \mathrm{E}-00 & 0 . \\ 139 & 327.6895561 & 0.342244 \mathrm{E}-00 & 0 . \\ 140 & 322.0341454 & 0.350911 \mathrm{E}-00 & 0 . \\ 141 & 316.4763374 & 0.375676 \mathrm{E}-00 & 0 . \\ 142 & 311.0144577 & 0.422946 \mathrm{E}-00 & 0 . \\ 143 & 305.6468315 & 0.452886 \mathrm{E}-00 & 0 . \\ 144 & 300.3718491 & 0.441973 \mathrm{E}-00 & 0 . \\ 145 & 295.1879005 & 0.420839 \mathrm{E}-00 & 0 . \\ 163 & 215.7776318 & 0.427905 \mathrm{E}-00 & 0 . \\ 164 & 212.0536461 & 0.430182 \mathrm{E}-00 & 0 . \\ 165 & 208.3939323 & 0.409888 \mathrm{E}-00 & 0 . \\ 166 & 204.7973824 & 0.381413 \mathrm{E}-00 & 0 . \\ 147 & 290.0934181 & 0.399542 \mathrm{E}-00 & 0 . \\ 148 & 285.0868607 & 0.394269 \mathrm{E}-00 & 0 . \\ 160 & 227.3465881 & 0.396054 \mathrm{E}-00 & 0 . \\ 149 & 275.3314705 & 0.383934 \mathrm{E}-00 & 0 . \\ 150 & 270.5796852 & 0.395527 \mathrm{E}-00 & 0 . \\ 151 & 265.9099007 & 0.444330 \mathrm{E}-00 & 0 . \\ 152 & 261.3207130 & 0.453028 \mathrm{E}-00 & 0 . \\ 153 & 256.8107224 & 0.421413 \mathrm{E}-00 & 0 . \\ 154 & 252.3785801 & 0.397593 \mathrm{E}-00 & 0 . \\ 155 & 248.0229168 & 0.386121 \mathrm{E}-00 & 0 . \\ 156 & 243.7424316 & 0.382382 \mathrm{E}-00 & 0 . \\ 157 & 239.5358181 & 0.389930 \mathrm{E}-00 & 0 .\end{array}$

$0.632724 E 00$ $0.630652 \mathrm{E} 00$ $0.623568 \mathrm{E} \quad 00$ $0.620465 E \quad 00$ $0.625259 \mathrm{E} 00$ $0.629305 E \quad 00$ $0.728101 \mathrm{E}$ DO 0.778910 E 00 $0.870464 E \quad 00$ $0.101218 \mathrm{E} \quad 1$ $0.118725 E \quad 01$ $0.142574 t \quad 01$ $0.158786 E \quad 01$ $0.165908 E$ OL $0.162203 E$ OI $0.143882 E \quad O 1$ $0.130573 E \quad 01$ $0.128331 \mathrm{E}$ O1 $0.124158 \mathrm{E}$ OI $0.117964 E$ OI $0.112364 E \quad 01$ $0.104914 \mathrm{E} \quad 01$ $0.990107 E$ OO $0.983768 t \quad 00$ $0.969852 \mathrm{t} \quad 00$ 0.918065 t 00 $0.954132 \mathrm{E} \quad 00$ $0.953038 t \quad 00$ $0.900474 t \quad 00$ $0.924634 \mathrm{E} \mathrm{OO}$ 0.936889 t 00 $0.924169 \mathrm{E} \mathrm{OO}$ 0.871955 E 00 $0.824467 \mathrm{E} \quad 00$ $0.819912 \mathrm{E} \quad 00$ $0.900632 \mathrm{E} \mathrm{OO}$ $0.912533 E \quad 00$ $0.925669 \mathrm{E} \mathrm{OO}$ $0.936920 \mathrm{E} \mathrm{OO}$ 0.966229 E 00 $0.973042 \mathrm{E} 00$ $0.913050 E$ OO $0.884426 \mathrm{E} \quad 00$ $0.910831 \mathrm{E} 00$ $0.930947 E \quad 00$ $0.943843 \mathrm{E} \quad 00$ $0.962691 \mathrm{E} \mathrm{OO}$ $0.964461 t \quad 00$ 0.943787 E CO 0.947204 E CO $0.952505 \mathrm{t} 00$ $0.945988 \mathrm{E} \quad 00$ $0.933445 \mathrm{E} \quad 00$ 0.904231 t 00 0.889755 t 00 0.901539 E 00 0.926095 t 00
$0.312597 E-00$ $0.313647 \mathrm{E}-00$ $0.314425 E-00$ $0.314982 E-00$ $0.315431 \mathrm{~F}-00$ $0.315829 E-00$ $0.319880 \mathrm{E}-00$ $0.324281 \mathrm{E}-00$ $0.329466 E-00$ $0.335506 E-00$ $0.342264 E-00$ $0.349564 E-00$ $0.356968 \mathrm{E}-00$ $0.364296 \mathrm{E}-00$ $0.371363 E-00$ $0.377885 E-00$ $0.383905 E-00$ $0.389661 \mathrm{E}-00$ $0.395055 E-00$ $0.400117 \mathrm{E}-00$ $0.404787 \mathrm{E}-00$ $0.408887 E-00$ $0.412403 E-00$ $0.415783 E-00$ $0.418793 \mathrm{E}-00$ $0.421077 \mathrm{E}-00$ $0.423548 E-00$ $0.425911 \mathrm{E}-00$ $0.427829 \mathrm{E}-00$ $0.429876 E-00$ $0.431668 \mathrm{E}-00$ $0.432933 E-00$ $0.433275 \mathrm{E}-00$ $0.432952 \mathrm{E}-00$ $0.432668 \mathrm{E}-00$ $0.432138 E-00$ $0.431858 \mathrm{E}-00$ $0.431564 E-00$ $0.431132 E-00$ $0.430822 E-00$ $0.430217 \mathrm{E}-00$ $0.428627 \mathrm{E}-00$ $0.426745 \mathrm{E}-00$ $0.425321 \mathrm{E}-00$ $0.424213 E-00$ $0.423207 \mathrm{E}-00$ $0.422182 \mathrm{E}-00$ $0.420937 \mathrm{E}-00$ $0.419316 E-00$ $0.41771 \mathrm{CE}-00$ $0.416069 \mathrm{E}-00$ $0.41432 \mathrm{CE}-00$ $0.412319 \mathrm{E}-00$ $0.409825 E-00$ $0.4 C 7188 \mathrm{E}-00$ $0.40482 \mathrm{CE}-00$ $0.402872 \mathrm{E}-00$
$0.65250 E-01$ $0.66918 E-01$ $0.69056 E-01$ $0.71504 E-01$ $0.74193 E-01$ $0.77005 E-01$ $0.79879 E-01$ $0.82516 E-01$ $0.84510 \mathrm{E}-01$ $0.86077 \mathrm{E}-01$ $0.86537 E-01$ $0.85466 \mathrm{E}-01$ $0.83027 \mathrm{E}-01$ $0.79524 \mathrm{E}-01$ $0.75339 E-01$ $0.70670 E-01$ $0.66335 \mathrm{E}-01$ $0.62381 E-01$ $0.59097 E-01$ $0.56405 \mathrm{E}-01$ $0.55549 E-01$ $0.54792 \mathrm{E}-01$ $0.55106 E-01$ $0.55110 \mathrm{E}-01$ $0.56013 \mathrm{E}-01$ $0.57087 E-01$ $0.57294 E-01$ $0.57334 \mathrm{E}-01$ $0.56272 \mathrm{E}-01$ $0.56592 \mathrm{E}-01$ $0.59776 E-01$ $0.63309 E-01$ $0.65302 E-01$ $0.65127 E-01$ $0.63341 \mathrm{E}-01$ $0.61626 E-01$ $0.60080 \mathrm{E}-01$ $0.60918 E-01$ $0.62843 E-01$ $0.64683 E-01$ $0.66991 \mathrm{E}-01$ $0.67438 E-01$ $0.67042 E-01$ $0.65741 \mathrm{E}-01$ $0.64413 E-01$ $0.64070 E-01$ $0.66140 E-01$ $0.67377 \mathrm{E}-01$ $0.68009 E-01$ $0.67721 E-01$ $0.67558 E-01$ $0.67045 E-01$ $0.67242 E-01$ $0.67699 \mathrm{E}-01$ C.66909E-01 $0.65321 E-01$ $0.63621 E-01$
$0.54261 \mathrm{E}-02$ $0.53325 \mathrm{E}-02$ $0.52404 \mathrm{E}-02 \quad 0$. $0.51500 \mathrm{E}-02$ 0. $0.50611 \mathrm{E}-02 \quad 0$. $0.49738 \mathrm{E}-02 \quad 0$. $0.81134 \mathrm{E}-02 \quad 0$. $0.79734 \mathrm{E}-02 \quad 0$. $0.78358 \mathrm{E}-02$ 0. $0.77006 \mathrm{E}-02 \quad 0$. $0.75677 \mathrm{E}-02$ O. $0.74371 E-02 \quad 0$. $0.73087 E-02 \quad 0$. $0.71826 \mathrm{E}-020$. $0.70586 \mathrm{E}-02 \quad 0$. $0.69368 \mathrm{E}-02 \quad 0$. $0.68171 \mathrm{E}-02$ 0. $0.66994 \mathrm{E}-02 \quad 0$. $0.65838 \mathrm{E}-02 \quad 0$. $0.64702 \mathrm{E}-02$ 0 . $0.63585 E-02 \quad 0$. $0.62488 \mathrm{E}-02$ 0. $0.61409 E-02 \quad 0$. $0.60349 \mathrm{E}-020$. $0.59308 \mathrm{E}-02 \quad 0$. $0.58284 E-02 \quad 0$. $0.57278 \mathrm{E}-02 \quad 0$. $0.56290 \mathrm{E}-02$ 0. $0.55318 \mathrm{E}-02 \quad 0$. $0.53426 E-02 \quad 0$. $0.52504 \mathrm{E}-02 \quad 0$. $0.51597 \mathrm{E}-02 \quad 0$. $0.50707 \mathrm{E}-02 \quad 0$. $0.49832 \mathrm{E}-02$ 0. $0.49070 E-020$. $0.48223 E-020$. $0.47391 \mathrm{E}-02$ 0. $0.46573 \mathrm{E}-02 \quad 0$. $0.45769 \mathrm{E}-02$ O. $0.44979 \mathrm{E}-02$ 0. $0.44203 \mathrm{E}-020$. $0.43440 E-02 \quad 0$. $0.42690 \mathrm{E}-02 \quad 0$. $0.41954 \mathrm{E}-02 \quad 0$. $0.41230 E-02$ O. $0.40518 \mathrm{E}-020$. $0.39819 \mathrm{E}-02 \quad 0$. $0.39131 \mathrm{E}-02 \quad 0$. $0.38456 \mathrm{E}-02$ 0 . $0.37792 \mathrm{E}-02$ 0 . $0.37140 \mathrm{E}-02$ 0 . $0.36499 \mathrm{E}-02 \quad 0$. $0.35869 E-02 \quad 0$. $0.35250 \mathrm{E}-02 \quad 0$. $0.34642 \mathrm{E}-02 \quad 0$. $0.34044 \mathrm{E}-02 \quad 0$.

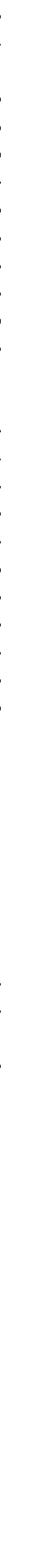




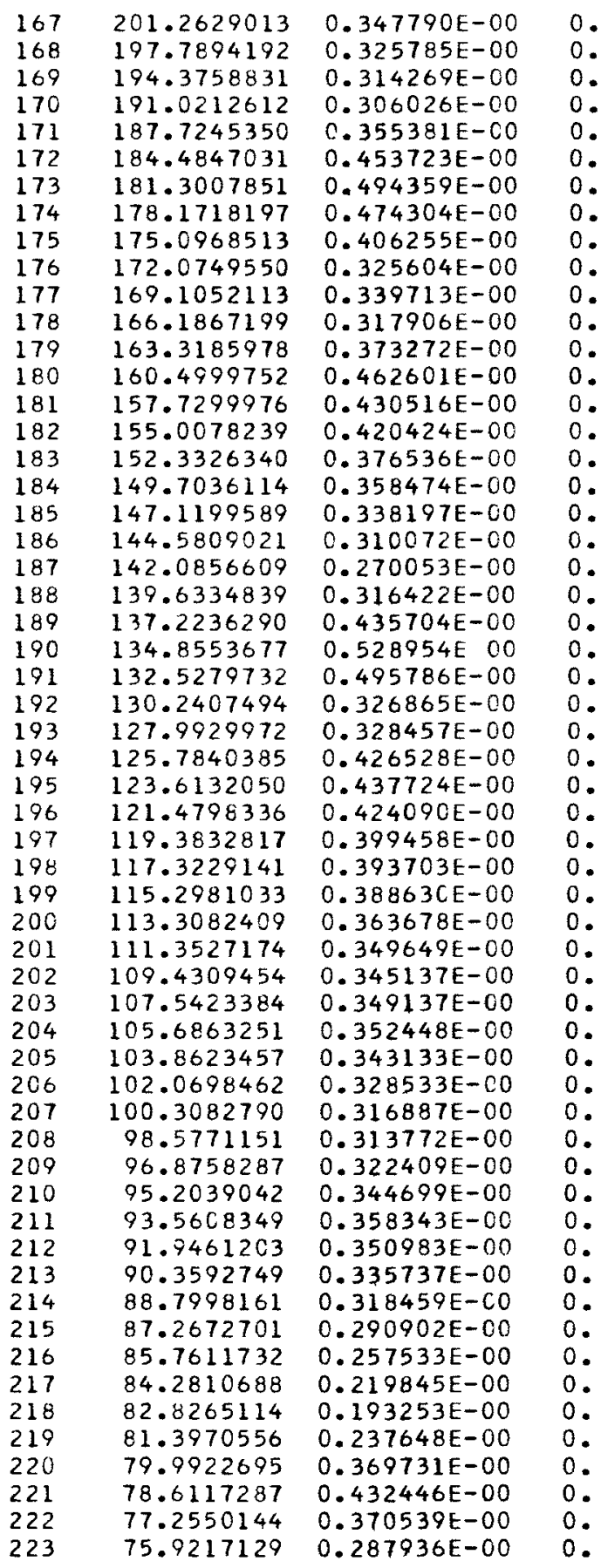

$0.973364 \mathrm{t} \quad 00 \quad 0.401444 \mathrm{E}-00 \quad 0.63161 \mathrm{E}-01$ $0.102511[01 \quad 0.400339 E-00$ 0.107511 t 01 $0.112691 \mathrm{E} 01$ $0.106844 \mathrm{E} \mathrm{Cl}$ $0.925222 E \quad 00$ 0.857347 E 00 $0.935955 E$ กO $0.971274 E \quad 00$ $0.107758 E \quad 01$ $0.109584 \mathrm{E} \quad 01$ $0.114393 \mathrm{E} \mathrm{C1}$ $0.110581 E \quad 01$ 0.966878 E 00 $0.979774 t \quad 00$ 0.993792 t 00 $0.101609 \mathrm{E} 01$ $0.105942 t \quad 01$ $0.111663 E$ OI $0.119619 \mathrm{E}$ O1 $0.134234 E \quad 01$ $0.128264 \mathrm{E} \quad 01$ 0.105501 t 01 $0.932686 E$ OO $0.925531 \mathrm{E}$ 0O $0.110806 E$ OL $0.113153 \mathrm{t} 01$ $0.960810 \mathrm{E} 00$ $0.940968 \mathrm{E} 00$ $0.958150 E$ OO 0.938164 E OO $0.928442 \mathrm{E} \quad 00$ 0.934382 E 00 $0.957248 \mathrm{E}$ OO $0.983003 \mathrm{E} 00$ $0.100427 \mathrm{E} 01$ $0.107339 \mathrm{E} 01$ $0.107104 \mathrm{E} \quad 01$ $0.108360 \mathrm{E}$ 01 $0.111084 \mathrm{E} 01$ $0.114108 \mathrm{E} \quad 01$ $0.115810 \mathrm{E}$ OI $0.114837 E \quad 01$ $0.110746 E$ OI $0.107139 E$ E 1 $0.106525 E \quad 01$ $0.106918 \mathrm{E} 01$ 0.107925 t 01 $0.112584 t \quad 01$ 0.121551 E 01 $0.136167 \mathrm{t} 01$ $0.153843 E \quad 01$ $0.143045 E$ O1 $0.110711 E$ OI $0.982752 \mathrm{E}$ OO 0.102010 E OI $0.108967 \mathrm{E} \quad 01$ 0.39937 AE-0O $0.3985 \mathrm{C} 7 \mathrm{E}-00$ $0.396721 E-30$ $0.393103 E-00$ $0.388637 \mathrm{E}-00$ $0.386726 \mathrm{E}-00$ $0.38591 \mathrm{CE}-00$ $0.386442 \mathrm{E}-0 \mathrm{D}$ $0.38664 \mathrm{CE}-00$ $0.387126 \mathrm{E}-00$ $0.386556 E-00$ $0.384238 \mathrm{E}-00$ $0.382382 \mathrm{E}-00$ $0.380614 \mathrm{E}-00$ $0.379516 E-00$ $0.378664 E-00$ $0.378099 \mathrm{E}-00$ $0.377958 E-00$ $0.378451 E-00$ $0.378069 E-00$ $0.375455 E-00$ $0.371023 \mathrm{E}-00$ $0.367066 E-00$ $0.366075 E-00$ C. $364990 E-00$ $0.362024 E-00$ $0.358764 E-00$ $0.355680 t-00$ $0.352922 t-00$ $0.350178 E-00$ $0.347455 E-00$ $0.345112 \mathrm{E}-00$ $0.342962 \mathrm{E}-00$ $0.340839 E-00$ $0.337976 \mathrm{E}-00$ $0.33499 C E-00$ $0.332118 E-00$ $0.329468 \mathrm{E}-00$ $0.326985 E-00$ $0.324509 \mathrm{E}-00$ $0.321816 \mathrm{E}-00$ $0.318642 E-00$ $0.315143 \mathrm{E}-00$ $0.311717 \mathrm{E}-\mathrm{CO}$ $0.308516 E-00$ $0.305581 E-00$ $0.303118 \mathrm{E}-00$ $0.301244 \mathrm{E}-00$ $0.300036 E-00$ $0.299285 \mathrm{E}-00$ $0.297689 \mathrm{E}-00$ $0.293559 \mathrm{E}-00$ $0.288140 \mathrm{E}-00$ $0.283836 E-00$ $0.28103 \mathrm{CE}-0 \mathrm{O}$
0.64339 $0.66865 \mathrm{E}-01$ $0.69771 \mathrm{E}-0$ $0.73458 E-01$ $0.75818 \mathrm{E}-01$ $0.74549 \mathrm{E}-01$ $0.70017 \mathrm{E}-01$ $0.64021 \mathrm{E}-01$ $0.61641 E-01$ $0.64315 \mathrm{E}-01$ $0.67385 \mathrm{E}-01$ $0.71026 \mathrm{E}-01$ $0.73895 \mathrm{E}-01$ $0.70265 E-01$ $0.70665 E-01$ $0.65580 E-01$ $0.67451 \mathrm{E}-01$ $0.67343 \mathrm{E}-01$ C. $71667 \mathrm{E}-01$ C. $74500 E-01$ $0.78710 E-01$ $0.82183 E-01$ $0.82203 E-01$ $0.77911 \mathrm{E}-01$ $0.69321 \mathrm{E}-01$ $0.67904 E-01$ $0.69169 \mathrm{E}-01$ $0.70384 \mathrm{E}-01$ $0.69339 \mathrm{E}-01$ $0.62951 E-01$ $0.60896 E-01$ $0.61110 \mathrm{~F}-01$ $0.59493 E-01$ $0.59823 E-01$ $0.61155 E-01$ $0.62819 E-01$ $0.63343 E-01$ $0.63099 E-01$ $0.63054 E-01$ $0.63588 \mathrm{E}-01$ $0.64402 \mathrm{E}-01$ $0.65140 E-01$ $0.65426 E-01$ $0.64682 E-01$ $0.62811 \mathrm{E}-01$ $0.60414 E-01$ $0.58250 E-01$ $0.57476 E-01$ $0.57610 \mathrm{E}-01$ $0.58831 \mathrm{E}-01$ $0.61465 \mathrm{E}-01$ $0.65604 \mathrm{E}-01$ $0.68973 E-01$ $0.68123 E-01$ $0.62458 E-01$ $0.54168 \mathrm{E}-01$
$.33456 E-020$. $0.32312 E-02$ O. $0.31754 \mathrm{E}-02 \quad 0$. $0.31206 E-02$ 0 . $0.30667 E-02$ O. $0.30138 E-02$ 0. $0.54352 E-02$ O. $0.53414 E-02$ $0.52493 E-02 \quad 0$. $0.51587 \mathrm{E}-02$ O $0.50696 \mathrm{E}-02$ O. $0.49821 \mathrm{E}-02 \quad 0$. $0.48962 \mathrm{E}-02 \quad 0$. $0.48117 \mathrm{E}-02 \quad 0$. $0.47286 \mathrm{E}-02$ 0 . $0.46470 E-02$. $0.45668 E-02$ O. $0.44880 \mathrm{E}-02$ O. $0.44105 E-02 \quad 0$. $0.43344 \mathrm{E}-02 \quad 0$. $0.42596 \mathrm{E}-02$ $0.41861 \mathrm{E}-02 \quad 0$. $0.41138 \mathrm{E}-02 \quad 0$. $0.40429 E-02 \quad 0$. $0.39731 \mathrm{~F}-\mathrm{C} 2 \mathrm{O}$ $0.38371 E-02$ O. $0.37709 E-02 \quad 0$. $0.37058 \mathrm{E}-02 \quad 0$. $0.36419 \mathrm{E}-02$ 0. $0.35790 \mathrm{~F}-02 \quad 0$ $0.34565 \mathrm{E}-02 \quad 0$. $0.33969 E-02 \quad 0$. $0.33383 \mathrm{E}-02 \quad 0$. $0.29379 \mathrm{E}-02$ 0 . $0.28872 \mathrm{E}-02$ 0. $0.28374 \mathrm{E}-02 \quad 0$. $0.27884 \mathrm{E}-02 \quad 0$. $0.27403 E-02 \quad 0$. $0.26930 \mathrm{E}-02$ 0 $0.26465 E-02 \quad 0$. $0.26008 \mathrm{E}-02 \quad 0$. 0.25560 E-02 0 . $0.25118 E-020$. $0.24685 E-02 \quad 0$. $0.24259 \mathrm{E}-02 \quad 0$. $0.23840 E-02 \quad 0$. $0.23429 E-02 \quad 0$. $0.23024 E-02 \quad 0$. $0.22627 \mathrm{E}-02$ 0. $0.22237 \mathrm{E}-02 \quad 0$. $0.21853 \mathrm{E}-02$ 0. $0.21476 \mathrm{E}-02$ 0 . $0.21105 E-02 \quad 0$ 


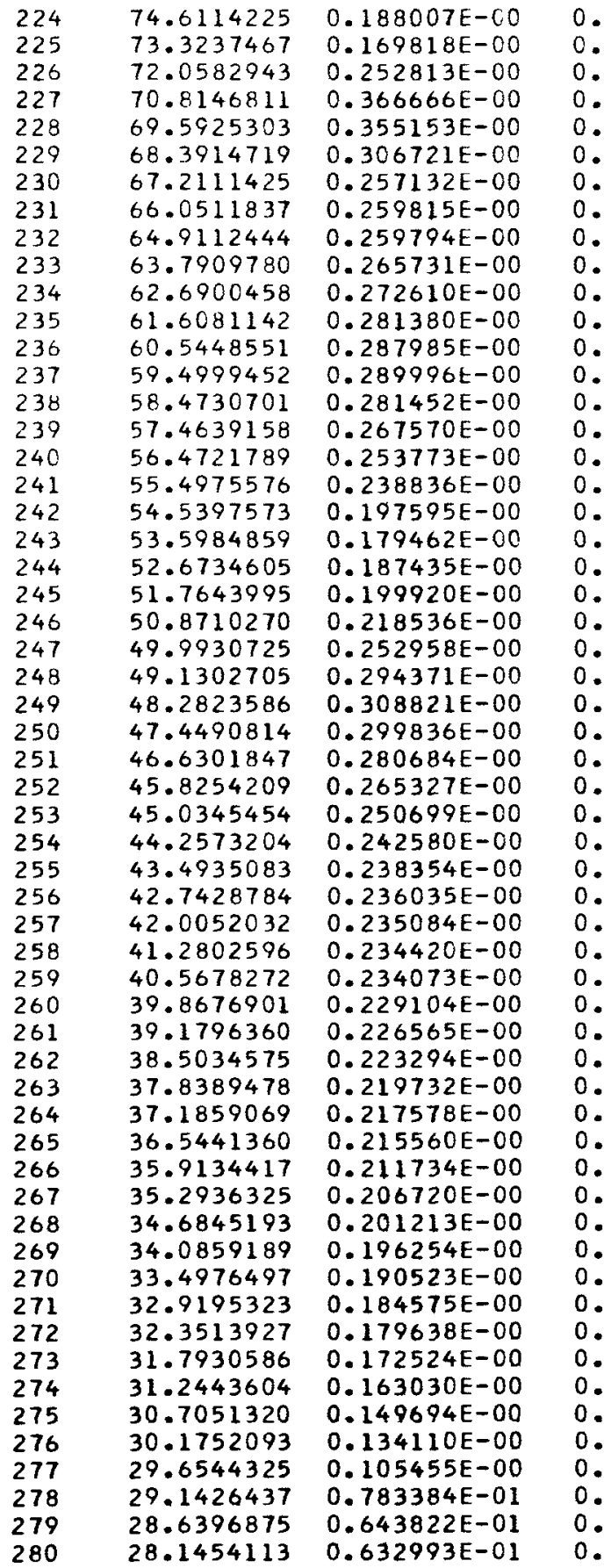

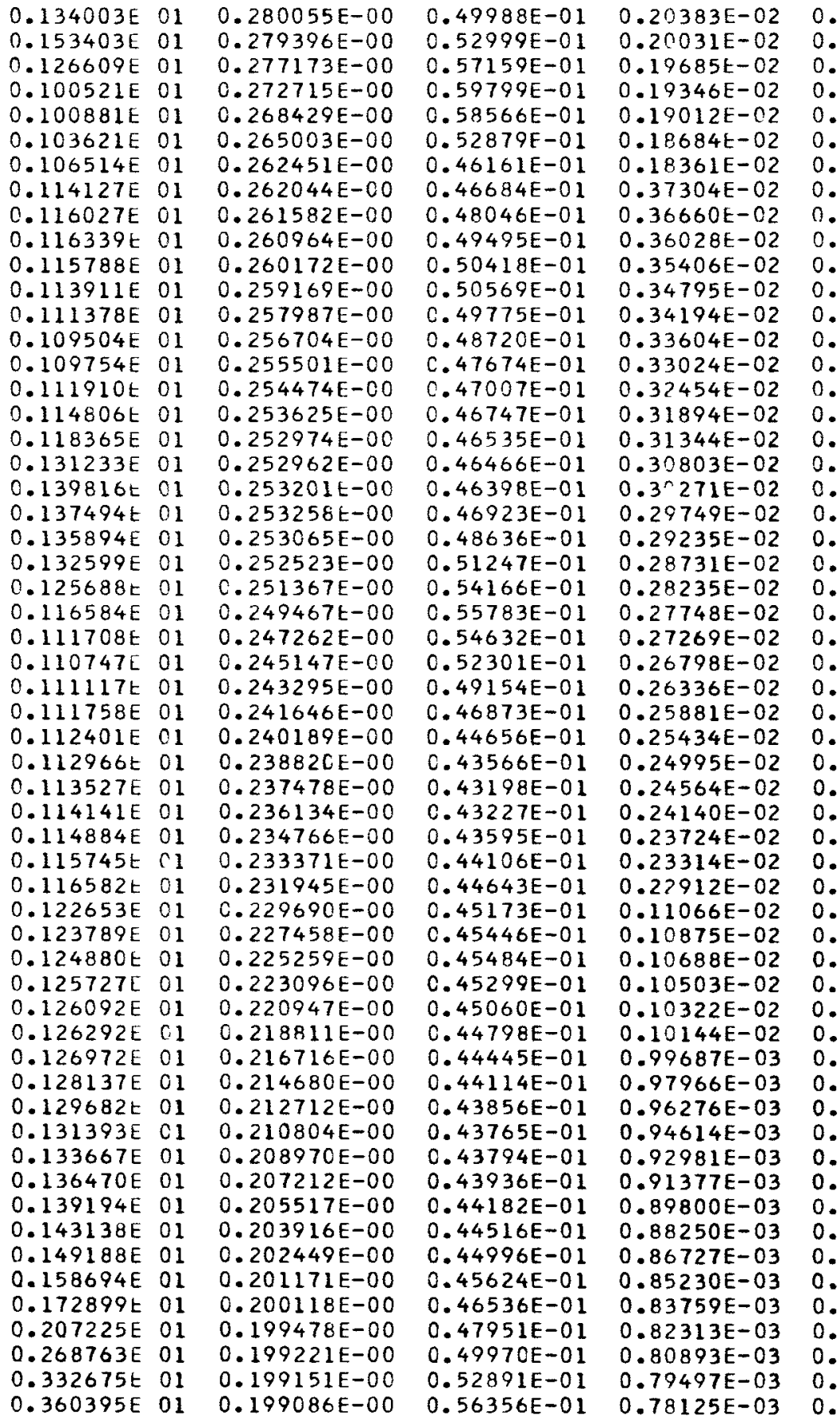




\begin{tabular}{|c|c|c|}
\hline $\begin{array}{l}281 \\
282\end{array}$ & $\begin{array}{l}27.6596653 \\
27.1823034\end{array}$ & $\begin{array}{l}0.683854 \mathrm{E}-01 \\
0.981148 \mathrm{E}-01\end{array}$ \\
\hline 283 & 26.7131793 & $0.179209 E-00$ \\
\hline 284 & 26.2521515 & $0.264369 E-00$ \\
\hline 285 & 25.7990806 & $0.313209 E-00$ \\
\hline 286 & 25.3538291 & $0.303747 \mathrm{E}-00$ \\
\hline 287 & 24.9162617 & $0.264866 \mathrm{E}-00$ \\
\hline 288 & 24.4862459 & $0.212268 E-00$ \\
\hline 289 & 24.0636516 & $0.177788 \mathrm{E}-\mathrm{CO}$ \\
\hline 290 & 23.6483510 & $0.161688 E-00$ \\
\hline 291 & 23.2402172 & $0.161080 E-00$ \\
\hline 292 & 22.8391273 & $0.164932 E-00$ \\
\hline 293 & 22.4449599 & $0.166911 \mathrm{E}-00$ \\
\hline 294 & 22.0575955 & $0.168297 \mathrm{E}-\mathrm{CO}$ \\
\hline 295 & 21.6769159 & $0.169577 \mathrm{E}-00$ \\
\hline 296 & 21.3028061 & $0.171456 \mathrm{E}-00$ \\
\hline 297 & 20.9351532 & $0.171904 \mathrm{E}-00$ \\
\hline 298 & 20.5738454 & $0.169697 E-C O$ \\
\hline 299 & 20.2187731 & $0.165578 E-00$ \\
\hline 300 & 19.8698287 & $0.160494 \mathrm{E}-00$ \\
\hline 301 & 19.5269065 & $0.155558 \mathrm{E}-00$ \\
\hline 302 & 19.1899028 & $0.150913 E-00$ \\
\hline 303 & 18.8587151 & $0.146615 \mathrm{E}-00$ \\
\hline 304 & 18.5332432 & $0.143156 \mathrm{E}-00$ \\
\hline 305 & 18.2133887 & $0.139830 E-00$ \\
\hline 306 & 17.8990541 & $0.136434 E-00$ \\
\hline 307 & 17.5901444 & $0.132905 E-00$ \\
\hline 308 & 17.2865663 & $0.129387 E-C O$ \\
\hline 309 & 16.9882271 & $0.126218 E-C 0$ \\
\hline 310 & 16.6950369 & $0.122501 \mathrm{E}-00$ \\
\hline 311 & 16.4069068 & $0.118648 E-00$ \\
\hline 312 & 16.1237493 & $0.115134 \mathrm{E}-00$ \\
\hline 313 & 15.8454785 & $0.112165 E-00$ \\
\hline 314 & 15.5720106 & $0.110771 E-00$ \\
\hline 315 & 15.3032621 & $0.111562 \mathrm{E}-\mathrm{CO}$ \\
\hline 316 & 15.0391518 & $C .114380 \mathrm{E}-\mathrm{CO}$ \\
\hline 317 & 14.7795994 & $0.118005 \mathrm{E}-00$ \\
\hline 318 & 14.5245271 & $0.122632 E-00$ \\
\hline 319 & 14.2738564 & $0.124854 E-C 0$ \\
\hline 320 & 14.0275121 & $0.122122 E-00$ \\
\hline 321 & 13.7854191 & $0.116855 E-00$ \\
\hline 322 & 13.5475044 & $0.111293 E-00$ \\
\hline 323 & 13.3136957 & $0.107010 E-00$ \\
\hline 324 & 13.0839221 & $0.104583 E-00$ \\
\hline 325 & 12.8581141 & $0.103177 \mathrm{E}-00$ \\
\hline $\begin{array}{l}326 \\
327\end{array}$ & 12.6362034 & $0.102792 \mathrm{E}-00$ \\
\hline $\begin{array}{l}327 \\
328\end{array}$ & $\begin{array}{l}12.4181222 \\
12.2038051\end{array}$ & $0.1 C 2862 \mathrm{E}-00$ \\
\hline $\begin{array}{l}328 \\
329\end{array}$ & $\begin{array}{l}12.2038051 \\
11.9931865\end{array}$ & $0.102390 E-C O$ \\
\hline $\begin{array}{l}329 \\
330\end{array}$ & $\begin{array}{l}11.9931865 \\
11.7862029\end{array}$ & $\begin{array}{l}0.101361 \mathrm{E}-\mathrm{CO} \\
0.100326 \mathrm{E}-00\end{array}$ \\
\hline $\begin{array}{l}330 \\
331\end{array}$ & $\begin{array}{l}11.6862029 \\
11.5827914\end{array}$ & $\begin{array}{l}0.100326 \mathrm{E}-00 \\
0.995157 \mathrm{E}-01\end{array}$ \\
\hline 332 & $\begin{array}{l}11.5827914 \\
11.3828907\end{array}$ & $0.983829 E-01$ \\
\hline 333 & 11.1864399 & $0.968340 E-01$ \\
\hline 334 & 10.9933795 & $0.948594 \mathrm{E}-\mathrm{Cl}_{1}$ \\
\hline 335 & 10.8036509 & $0.928281 \mathrm{E}-01$ \\
\hline 336 & 10.6171968 & $0.904102 E-01$ \\
\hline 337 & 10.4339607 & $0.868752 E-01$ \\
\hline
\end{tabular}

$\begin{array}{llll}0.358816 E & 01 & 0.198939 E-00 & 0.60647 E-01\end{array}$ $0.288457 \mathrm{E}$ O1 $0.198364 \mathrm{E}-00 \quad 0.63959 \mathrm{E}-01$ $0.186191 \mathrm{t} 01$ $0.140843 E$ O 1 $0.121825 E$ OI $0.111605 \mathrm{E} \mathrm{Cl}$ $0.107415 E$ OI $0.111719 \mathrm{E}$ OI $0.113656 \mathrm{t}$ O $0.114871\left[\begin{array}{ll}0 \\ 0.115183 E & 01\end{array}\right.$ $0.115183 \mathrm{E}$ OI $0.115009 \mathrm{E}$ OI 0.115547 t 01 $0.116519 E$ OI $0.117513 \mathrm{E}$ OI $0.118414 \mathrm{E} 01$ $0.119352 t \quad 01$ 0.120296501 $0.121044 E$ OI 0.121690 E 01 $0.122317 E$ ? 1 $0.123138 \mathrm{E}$ OI $0.124333 E$ O $0.125670 t$ o $0.125670 \mathrm{t} 01$ $0.129158 \mathrm{t} \quad$ $0.131333 E$ OI C.133736t ? $0.136185 \mathrm{t}$ ol $0.139078 E$ OI C. $142370 \mathrm{E}$ OI 0.145621 t 01 $0.148703 E$ OI $0.150783 E$ OI $0.150889 t^{01}$ $0.148947[01$ $0.148778 E \quad 01$ $0.144271 E$ OI $0.140493 E_{01}$ $0.139286 t$ t $0.139756 t \quad 01$ 0.140890 t 01 $0.142242 E \quad 01$ $0.143063 t \quad 01$ $0.143381 \mathrm{E} 01$ $0.143138 \bar{E} \mathrm{C}$ 0.142420 t 01 $0.141926 E \quad 01$ $0.141740 \mathrm{t}$ O $0.141430 \mathrm{E}$ OI 0.140871 t 01 $0.140440 E \quad 01$ 0.140213 E 01 $0.140231 \mathrm{t} 01$ $0.140469 E$ OI $0.141215 E$ OI $0.143272 \mathrm{E} \quad 1$
$0.196596 E-00 \quad 0.66320 E-01$ $0.193514 \mathrm{E}-00 \quad 0.65215 \mathrm{E}-01$ $0.18963 C E-00 \quad 0.60783 E-01$ $0.185819 \mathrm{E}-00 \quad 0.50142 \mathrm{E}-01$ $0.182551 E-00 \quad 0.40815 E-01$ $0.179758 E-00 \quad 0.33660 E-01$ $0.177592 E-00 \quad 0.28993 E-01$ $0.175709 \mathrm{E}-0 \mathrm{O} \quad 0.26853 \mathrm{E}-01$ $0.173819 E-00 \quad 0.26857 E-01$ $0.171838 E-00 \quad 0.27485 E-01$ $0.169804 E-00 \quad 0.28135 E-01$ $0.167729 E-00 \quad 0.28878 E-01$ $0.165615 \mathrm{E}-00 \quad 0.29613 \mathrm{E}-01$ $0.163452 \mathrm{E}-00 \quad 0.30418 \mathrm{E}-01$ $0.161266 E-0 C \quad 0.30990 E-01$ $0.159106 E-00$ $0.157008 E-00$ $0.15498 E E-00$ C. $153045 \mathrm{E}-00$ $0.151173 \mathrm{E}-00$ $0.149368 E-00$ $0.147613 \mathrm{E}-\mathrm{CO}$ $0.145908 \mathrm{E}-00$ $0.144254 E-00$ $0.142634 E-00$ C. $141109 E-O C$ $0.139611 E-00$ $0.138172 E-00$ $0.1367935-00$ $0.13547 C E-00$ $0.134191 \mathrm{E}-00$ $0.132926 E-00$ $0.131635 \mathrm{E}-00$ $0.130279 E-00$ $0.128731 E-00$ $0.127082 E-00$ $0.125377 F-00$ $0.123710 \mathrm{E}-0 \mathrm{O}$ $0.12213 \mathrm{CE}-0$ $0.120645 E-00$ $0.11923 \mathrm{CE}-00$ $0.117851 E-00$ $0.116488 \mathrm{E}-0 \mathrm{C}$ C. $115124 \mathrm{E}-00$ $0.113748 E-00$ $0.112372 \mathrm{E}-00$ $0.111006 \mathrm{E}-00$ $0.10965 C E-0 O$ $0.10830 \mathrm{CE}-00$ C. $106961 \mathrm{E}-00$ $0.105643 \mathrm{E}-00$ $0.104354 E-00$ $0.103094 E-00$ $0.101871 \mathrm{E}-00$ $0.100708 E-00$
$0.76777 E-03$ $0.75451 E-03$ $0.74149 \mathrm{E}-03 \quad 0$ $0.72870 E-03 \quad 0$ $0.71612 \mathrm{E}-03 \quad 0$. $0.7^{\cap} 376 E-03 \quad 0$. $0.69162 t-03 \quad 0$. $0.11498 E-02 \quad 0$. $0.11300 \mathrm{E}-02 \quad 0$. $0.11105 E-02 \quad 0$. $0.1 C 913 \mathrm{E}-02$ O. $0.10725 \mathrm{E}-02 \quad 0$ $0.10358 E-02 \quad 0$. $0.10179 \mathrm{E}-02 \quad 0$. $0.10003 \mathrm{E}-02 \quad 0$. $0.98308 \mathrm{E}-03 \quad 0$ $0.96611 E-03$ O. $0.94944 \mathrm{E}-03 \quad 0$ $0.93305 E-030$. $0.91695 E-03 \quad 0$. $0.90112 E-03$ O. $0.88557 E-03$ O $0.88557 E-03$ O. $0.87029 \mathrm{E}-03 \quad 0$ $0.85527 E-030$. $0.84051 \mathrm{E}-03 \quad 0$. $0.82600 E-03 \quad 0$. $0.81175 E-03$ O $0.81175 E-03$ O. $0.79774 \mathrm{E}-03 \quad 0$ $0.78397 \mathrm{E}-03 \quad 0$. $0.77044 \mathrm{t}-03 \quad 0$. $0.75714 E-03 \quad 0$. $0.74408 E-03 \quad 0$ $0.73123 \mathrm{E}-03 \quad 0$. $0.71861 E-03 \quad 0$. $0.70621 \mathrm{E}-03 \quad 0$. $0.60456 E-03 \quad 0$. $0.59412 \mathrm{E}-03 \quad 0$. $0.58387 \mathrm{E}-03 \quad 0$. $0.57379 t-03 \quad 0$. $0.56389 \mathrm{E}-03 \quad 0$. $0.55416 \mathrm{E}-03$ O. $0.54459 E-030$. $0.53519 E-03 \quad 0$. $\begin{array}{ll}0.53519 E-03 & 0 . \\ 0.52596 E-03 & 0 .\end{array}$ $0.51688 E-030$. $0.5 C 796 t-03 \quad 0$. $0.49919 \mathrm{E}-03 \quad 0$. $0.49058 \mathrm{E}-03 \quad 0$. $0.4 P 211 \mathrm{E}-030$. $0.47379 E-03 \quad 0$. $0.46561 \mathrm{E}-030$. $0.45758 E-030$. $0.44968 E-030$. $0.44192 \mathrm{E}-03 \quad 0$. $0.43429 E-03 \quad 0$. $0.42680 E-030$. 


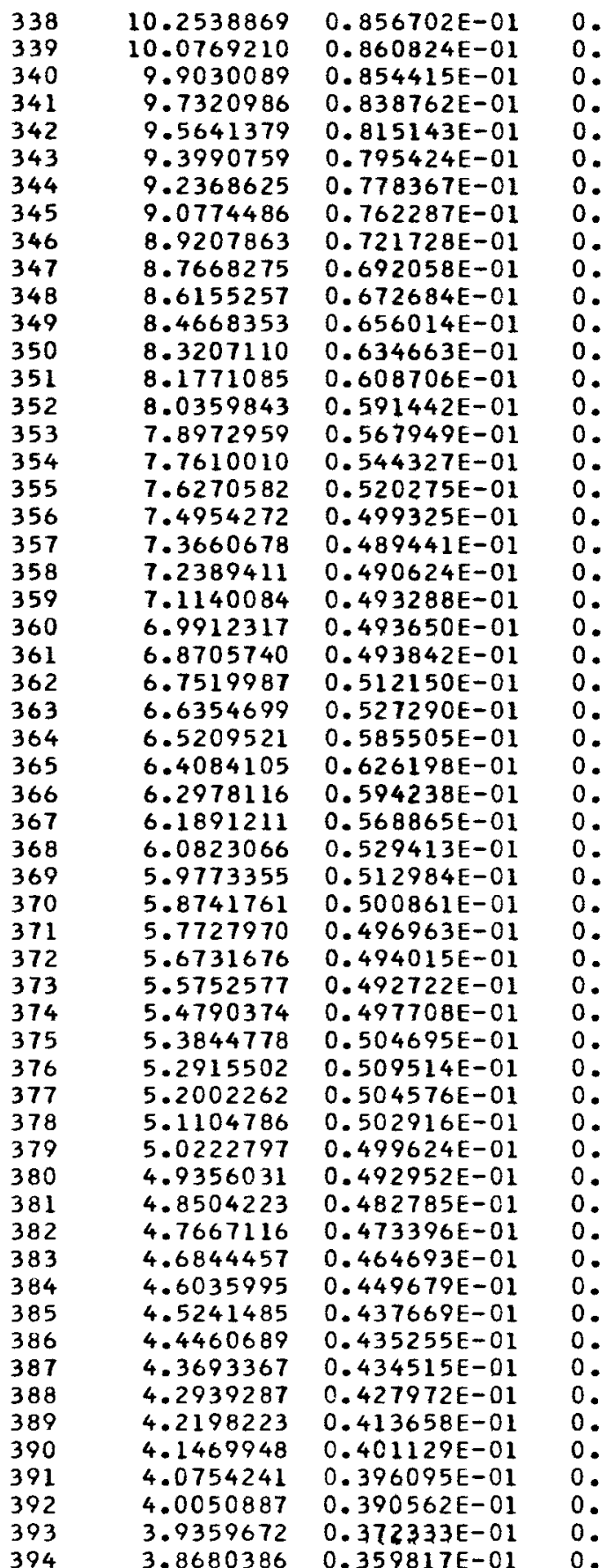

$0.143999 E \quad 01$ $0.142968 \mathrm{E} 01$ 0.142907 E 01 0.143677 E 01 0.144765 E 01 $0.145948 \mathrm{E}$ OI $0.147167 t \quad 01$ $0.148395 E$ O1 $0.152294 E 01$ $0.156364 \mathrm{E} \mathrm{OI}$ $0.159162 t$ ol $0.162301 \mathrm{E} \mathrm{O1}$ $0.166583 \mathrm{E} 01$ 0.171760 E 01 $0.176193 \mathrm{E} 01$ $0.181958 \mathrm{E} \quad 01$ $0.188892 \mathrm{E}$ OI $0.196209 \mathrm{E} 01$ $0.203749 \mathrm{E} 01$ 0.209093 E 01 $0.210814 \mathrm{E} 01$ 0.211345 t 01 0.211976 E 01 $0.211835 \mathrm{E} \mathrm{O1}$ $0.206402 \mathrm{E} 01$ $0.200473 E$ O1 $0.186371 \mathrm{E}$ O1 0.173410 E 01 $0.174259 \mathrm{E} 01$ $0.174948 \mathrm{E} \mathrm{OI}$ $0.176673 \mathrm{E}$ ol 0.179020 E 01 $0.180134 E$ E1 0.179855 E 01 0.179828 E 01 $0.179642 \mathrm{E} 01$ $0.177751 \mathrm{E} 01$ $0.175165 \mathrm{E} 01$ $0.172941 \mathrm{E}$ O1 0.172205 t 01 0.170838 E 01 $0.169186 t \quad 01$ $0.168324 t \quad 01$ $0.168119 E$ OI $0.167809 \mathrm{E} 01$ 0.167631 t 01 0.169279 E 01 0.170961 E 01 $0.170501 E 01$ $0.169636 t 01$ $0.170288 \mathrm{E}$ O1 $0.172571 \mathrm{E}$ O1 0.174731 E 01 0.175320 E 01 0.175918 E 01 $0.183898 \mathrm{E}$ o1 0.186061 E 01
$0.995603 E-01$ $0.983968 \mathrm{E}-01$ $0.972379 E-01$ $0.961014 \mathrm{E}-01$ $0.950022 \mathrm{E}-01$ $0.939335 \mathrm{E}-01$ $0.928903 E-01$ $0.918710 E-01$ $0.908935 E-01$ $0.899648 E-01$ $0.890663 \mathrm{E}-01$ $0.881937 E-01$ $0.873553 \mathrm{E}-01$ $0.865590 \mathrm{E}-01$ $0.857898 \mathrm{E}-01$ $0.850584 \mathrm{E}-01$ $0.843651 \mathrm{E}-01$ C. $8371 C 3 E-01$ $0.830889 E-01$ $0.824817 \mathrm{E}-01$ $0.818697 E-01$ $0.812502 \mathrm{E}-01$ 0.806270 E-01 $0.800003 \mathrm{E}-01$ $0.79339 \mathrm{CE}-01$ $0.786480 \mathrm{E}-01$ $0.778532 \mathrm{E}-01$ $0.769821 \mathrm{E}-01$ $0.761617 E-01$ $0.753807 \mathrm{E}-01$ $0.746632 \mathrm{E}-01$ $0.73971 \mathrm{CE}-01$ $0.732966 t-01$ $0.726258 \mathrm{E}-01$ $0.719574 \mathrm{E}-01$ $0.712884 \mathrm{E}-01$ $0.706079 E-01$ $0.699124 \mathrm{E}-01$ $0.692055 \mathrm{E}-01$ $0.685041 \mathrm{E}-01$ $0.678025 \mathrm{E}-01$ $0.671034 \mathrm{E}-01$ $0.664128 \mathrm{E}-01$ 0.657367 [-01 $0.65074 C E-01$ $0.644235 E-01$ $0.637965 \mathrm{E}-01$ $0.631881 \mathrm{E}-01$ $0.625814 E-01$ $0.619736 \mathrm{E}-\mathrm{Cl}$ $0.613749 \mathrm{E}-01$ $0.607989 \mathrm{E}-01$ $0.602425 E-01$ $0.596927 \mathrm{E}-01$ $0.591504 \mathrm{E}-01$ $0.583396 \mathrm{E}-01$ $0.575589 E-01$
$0.20944 E-01$ $0.20815 E-01$ 0.20676 E-01 $0.20500 E-01$ $0.20186 \mathrm{E}-01$ $0.19977 \mathrm{E}-01$ $0.19830 E-01$ $0.19698 \mathrm{E}-01$ $0.19575 E-01$ C. $19518 E-01$ $0.19557 \mathrm{E}-01$ $0.19651 \mathrm{E}-01$ $0.19862 \mathrm{E}-01$ $0.19941 E-01$ $0.20153 E-01$ $0.20333 E-01$ $0.20596 E-01$ $0.20803 E-01$ $0.21068 E-01$ $0.21373 E-01$ $0.21649 E-01$ $0.21853 E-01$ $0.21964 E-01$ $0.21942 \mathrm{E}-01$ $0.21849 \mathrm{E}-01$ $0.21683 E-01$ $0.21369 E-01$ $0.20856 \mathrm{~F}-01$ C. $19947 \mathrm{E}-01$ $0.19196 \mathrm{E}-01$ $0.18163 E-01$ $0.17931 \mathrm{E}-01$ $0.17670 E-01$ $0.17471 E-01$ $0.17397 \mathrm{E}-01$ $0.17296 \mathrm{E}-01$ $0.17197 \mathrm{E}-01$ $0.1705 C E-01$ $0.16906 \mathrm{E}-01$ $0.16650 \mathrm{E}-01$ $0.16372 \mathrm{E}-01$ C. $16050 \mathrm{E}-01$ $0.15723 E-01$ $0.15391 E-01$ C. $15039 \mathrm{E}-01$ $0.14762 \mathrm{E}-01$ $0.14525 E-01$ $0.14345 \mathrm{E}-01$ $0.14191 \mathrm{E}-01$ $0.14084 E-01$ $0.13970 E-01$ $0.13809 \mathrm{E}-01$ $0.13633 E-01$

$0.13533 \mathrm{E}-01$

$0.13431 E-01$

$0.13329 E-01$

$0.13119 \mathrm{E}-01$
$0.41943 E-03 \quad 0$ $0.41219 \mathrm{E}-03 \quad 0$. $0.40508 E-03 \quad 0$. $0.39809 E-03 \quad 0$. $0.39122 \mathrm{E}-030$. $0.38447 \mathrm{E}-03 \quad 0$. $0.37783 E-030$. $0.37131 \mathrm{E}-030$. 0.23477 E-03 0 . $0.23072 \mathrm{E}-03 \quad 0$. $0.22282 \mathrm{E}-030$. $0.21898 E-03 \quad 0$. $0.21520 \mathrm{E}-03 \quad 0$. $0.21148 E-030$. $0.20783 E-030$. $0.20425 \mathrm{E}-030$. $0.20072 E-030$. $0.19726 \mathrm{E}-03 \quad 0$. $0.19385 \mathrm{E}-030$. $0.19051 \mathrm{E}-030$. $0.18722 \mathrm{E}-03 \quad 0$. $0.18399 \mathrm{E}-03 \quad 0$. $0.18081 \mathrm{E}-03 \quad 0$. $0.17769 \mathrm{E}-03 \quad 0$. $0.17463 E-03 \quad 0$. $0.17161 \mathrm{E}-030$. $0.16865 E-03 \quad 0$. $0.16574 E-030$. $0.16288 \mathrm{E}-03 \quad 0$. $0.16007 E-030$. 0.15731 E-03 0 . $0.15459 \mathrm{E}-03 \quad 0$. $0.15192 \mathrm{E}-03 \quad 0$. $0.14930 \mathrm{E}-030$. $0.14672 \mathrm{E}-03 \quad 0$. $0.14419 \mathrm{E}-030$. $0.14170 \mathrm{E}-03 \quad 0$. $0.13926 E-03 \quad 0$. $0.13685 \mathrm{E}-03 \quad 0$. $0.13449 \mathrm{E}-030$ $0.13217 \mathrm{E}-030$. $0.12989 \mathrm{E}-03 \mathrm{O}$ $0.12765 \mathrm{E}-030$. $0.12545 \mathrm{E}-03 \quad 0$. $0.12328 \mathrm{E}-030$. $0.12115 \mathrm{E}-03 \quad 0$. $0.11906 \mathrm{E}-03 \quad 0$. $0.11701 \mathrm{E}-03 \quad 0$. $0.11499 E-03 \quad 0$. $0.11300 \mathrm{E}-030$. $0.11105 \mathrm{E}-03 \quad 0$. $0.10914 \mathrm{E}-03 \quad 0$. $0.10725 \mathrm{E}-03 \quad 0$. $0.10540 E-03 \quad 0$. $0.14947 E-03 \quad 0$. $0.14689 E-03$ 
3.0846226

3.0313869

2.9790698

2.9276558

2.8771290

2.8274743

2.7786765

2.7307209

2.6835930

2.6372783

2.5917630

2.5470333

2.5030755

2.4598763

2.4174227

2.3757018

2.3347009

2.2944076

2.2548098

2.2158953

2.1776524

2.1400695

2.1031353

2.0668385

2.0311681

1.9961133

1.9616635

1.9278083

1.8945373

1.8618406

1.8297082

1.7981303

1.7670974

1.7366000

1.7066290

1.6771753

1.6482299

1.6197840

$29804 E-01$

$0.117177 \mathrm{E}-01$

$0.963392 \mathrm{E}-02$

$0.745644 \mathrm{E}-02$

$0.600245 E-02$

$0.633606 \mathrm{E}-02$

$0.868405 \mathrm{E}-02$

$0.124737 \mathrm{E}-\mathrm{Cl}$

$0.153275 E-01$

$0.166555 \mathrm{E}-01$

$0.214431 \mathrm{E}-01$

$0.288175 \mathrm{E}-01$

$0.334819 E-01$

$0.356898 \mathrm{E}-01$

$0.372370 E-01$

$0.375131 E-01$

$0.369569 E-01$

$0.360362 E-01$

$0.354834 E-01$

$0.343457 E-01$

$0.330027 \mathrm{E}-01$

$0.322587 \mathrm{E}-01$

$0.318214 E-01$

$0.314282 \mathrm{E}-01$

$0.310650 \mathrm{E}-01$

$0.307141 \mathrm{t}-01$

$0.303145 \mathrm{E}-01$

$0.298073 \mathrm{E}-01$

$0.292741 \mathrm{E}-01$

$0.287472 \mathrm{E}-01$

$0.282091 E-01$

$0.276642 \mathrm{E}-01$

$0.271253 E-01$

$0.265657 \mathrm{E}-01$

$0.260412 \mathrm{E}-01$

$0.255768 \mathrm{E}-01$

C. $251008 \mathrm{E}-01$

$0.246492 \mathrm{E}-01$

$0.242278 E-01$

$\begin{array}{ll}1.5643566 & 0.233534 \mathrm{E}-\mathrm{C} 1\end{array}$

$1.5373583 \quad 0.229201 E-01$

$1.5108259 \quad 0.22478 \mathrm{CE}-\mathrm{CI}$

$1.48475140 .220420 \mathrm{E}-01$

$\begin{array}{ll}1.4591269 & 0.216018 \mathrm{E}-01 \\ 1.4339447 & 0.211623 \mathrm{E}-01\end{array}$ 0649-0 $0.225538 \mathrm{E} 01$ $0.241824 E \quad 01$ $0.252199 \mathrm{E} C 1$ 0.261658 t 01 $0.279692 \mathrm{E} \quad 01$ $0.295334 \mathrm{E}$ OI

$0.314822 \mathrm{E}$ OI $0.340995 E$ OI $0.396873 \mathrm{E} \mathrm{OI}$ $0.489745 \mathrm{E} C \mathrm{C}$ $0.586901 \mathrm{E} \mathrm{OI}$ $0.560142 \mathrm{t} \quad 01$ $0.424895 \mathrm{E} \quad$ OI $0.318330 \mathrm{E}$ OI $0.272991 E$ OI 0.260695 E 01

$0.222108 \mathrm{E} \quad 01$

$0.184501 E$ OI $0.170010 \mathrm{E}$ O $0.164729 E$ OI 0.161012 E 01 $0.158314 E \quad 01$ 0.157166 E OI $0.156528 E$ OI $0.155714 E \quad 0$ $0.155101 t$ OI $0.154694 \mathrm{E} 01$ $0.154236 E$ OI $0.153804 E$ OI $0.153553 E$ OI $0.153504 E$ OI $0.153515 \mathrm{E} 01$ $0.153455 E$ OI $0.153466 E$ OI 0.153477 E O1 $0.153366 E$ OI $0.153261 E$ OI 0.153220 E OI $0.153196 E^{O}$ $0.153295 E$ OI 0.153417 E OI $0.153426 \mathrm{E} \mathrm{OI}$ C.153516E OI $0.153605 E$ OI $0.153568 E$ OI $0.153593 \mathrm{E} \mathrm{OI}$ 0.153649 E 01 0.153659 t 01 $0.153715 \mathrm{E} O$ $0.153789 \mathrm{E}$ OI $0.153881 \mathrm{E} 01$

$12476 \mathrm{E}$

$12223 E-01$

$0.11910 E-01$

$0.11539 E-01$

$0.11156 \mathrm{E}-01$

$0.10800 E-01$

$0.10525 \mathrm{E}-01$

$0.10334 E-01$

$.10222 \mathrm{E}-01$

$0.10165 E-01$

$0.10147 \mathrm{E}-01$

$0.10084 E-01$

$0.99922 E-02$

$0.98617 E-02$

$0.97666 E-02$

$0.97979 E-02$

$0.99397 E-02$

$0.10110 \mathrm{E}-01$

$0.10307 E-01$

$0.10455 \mathrm{E}-01$

. $10641 E-01$

$0.10749 E-01$

$0.10774 \mathrm{E}-01$

$0.10535 \mathrm{E}-01$

$0.10247 E-01$

$0.99143 \mathrm{E}-02$

$0.96679 \mathrm{E}-02$

$0.92888 \mathrm{E}-02$

$0.88801 \mathrm{E}-02$

$0.86300 E-02$

$0.84689 \mathrm{E}-02$

$0.83390 \mathrm{E}-02$

$0.82383 \mathrm{E}-02$

$0.81445 \mathrm{E}-02$

$0.80325 E-02$

$0.78989 \mathrm{E}-02$

$0.77566 \mathrm{E}-02$

$0.76056 \mathrm{E}-02$

$.74533 E-02$

$0.73048 E-02$

C. $71596 \mathrm{E}-02$

$0.70197 \mathrm{E}-02$

C. $68883 \mathrm{E}-02$

$0.67648 E-02$

$0.65305 E-02$

$0.64151 \mathrm{E}-02$

C. $63004 \mathrm{E}-02$

$0.61881 E-02$

$0.50509 E-02$

$0.58483 E-02$

$0.57374 \mathrm{E}-02$

$0.56289 E-02$

14186E-03 0

$13941 \mathrm{E}-030$

$.13701 \mathrm{E}-03 \quad 0$

$0.13464 \mathrm{E}-03 \quad 0$.

$.13232 \mathrm{E}-03 \quad 0$.

$0.13003 \mathrm{E}-030$.

$0.12779 E-030$.

$0.12558 \mathrm{E}-030$.

$0.12342 \mathrm{E}-030$.

$0.12129 \mathrm{E}-030$.

$.11919 \mathrm{E}-030$.

$0.11714 E-03 \quad 0$.

$0.11512 \mathrm{E}-03 \quad 0$.

$0.11313 E-030$.

$.11118 \mathrm{E}-030$.

$0.10926 E-030$.

$0.10737 \mathrm{E}-030$.

$0.10552 E-030$.

$0.10370 E-030$. 


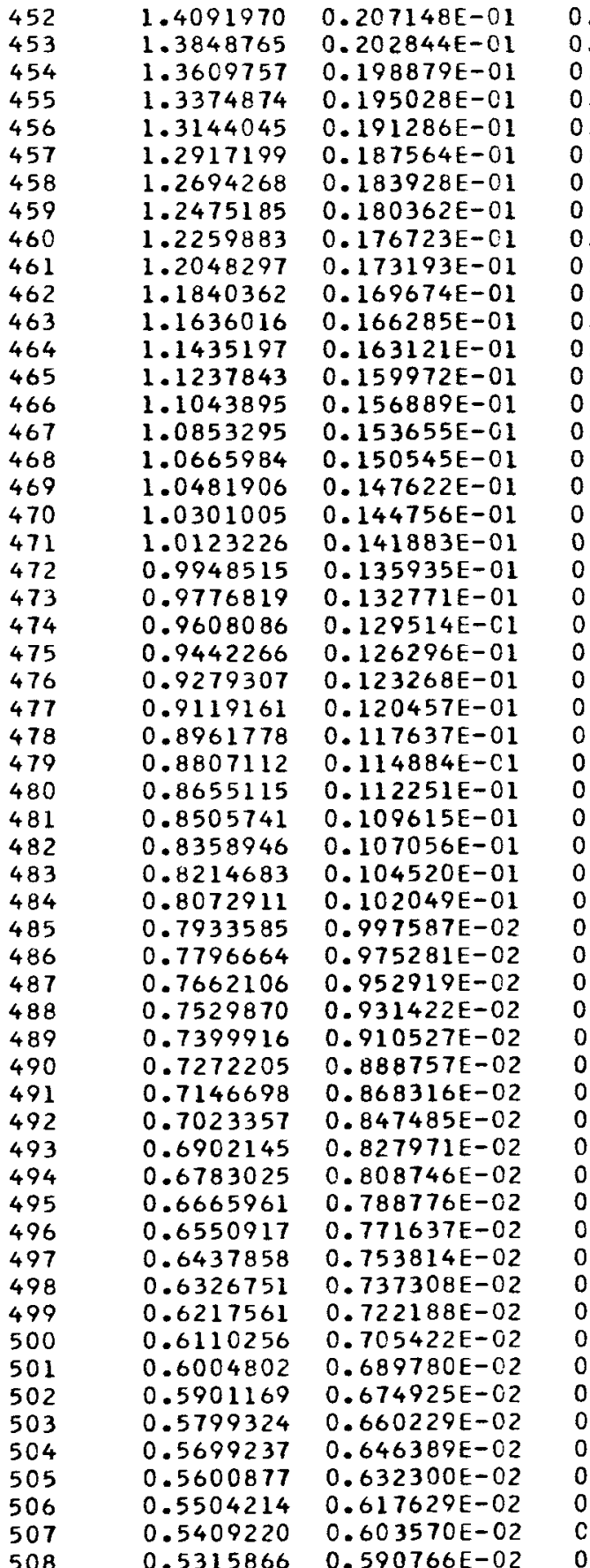

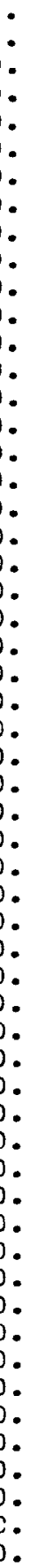

$0.154238 E \quad 01$ $0.154457 \mathrm{E}$ OI 0.154595 E 01 $0.154733 E$ OL $0.154870 \mathrm{t}$ OI $0.155008 \mathrm{E}$ OI $0.155008 E$ E $0.155146 E$ OI 0.155483 E 01 $0.155682 \mathrm{E} \quad 01$ $0.155901 \mathrm{E}$ O1 $0.156120 E \quad 01$ 0.156257 t $\mathrm{Cl}$ $0.156401 E$ OL $0.156557 \mathrm{E}$ OI $0.156801 \mathrm{E} 01$ $0.157044 E$ OI $0.157206 E$ OI $0.157368 E$ OI $0.157576 t$ OI 0.176017 t 01 0.176179 E 01 $0.176407 \mathrm{E}$ OI $0.176615 E$ OI $0.176778 \mathrm{E} \quad$ $0.176940 \mathrm{E} 01$ $0.177148 \mathrm{t} \quad$ $0.177356 E$ OI $0.177518 E$ OI $0.177726 \mathrm{t}$ ol $0.177934 \mathrm{E} \quad 01$ $0.178142 \mathrm{t} \quad 01$ $0.178350 \mathrm{E}$ OI $0.178431 \mathrm{E}$ OI $0.178477 \mathrm{E}$ O $0.178603 E$ OI $0.178684 \mathrm{E} \quad 01$ 0.178725 t 01 0.178927 E OI 0.179089 E OI 0.179297 t 01 0.179505 E OI $0.179713 \mathrm{t}$ O $0.180083 E$ OL $0.180245 E$ OI $0.180453 \mathrm{E}$ O 0.180661 t 01 $0.180661 E$ OI $0.180869 t \quad 01$ $0.181077 \mathrm{E} \quad 0$ $0.181123 E \quad 01$ $0.181215 E$ OI $0.181261 E$ O 1 $0.181514 E$ OI $0.181722 t \quad 01$ $0.181768 \mathrm{E}$ ol
$0.248142 \mathrm{E}-\mathrm{O} 1$ $.243397 \mathrm{E}-01$ $0.238745 \mathrm{E}-01$ $0.234185 E-01$ $0.229714 \mathrm{E}-01$ $0.225331 \mathrm{E}-01$ $0.221035 E-01$ $0.216823 \mathrm{E}-01$ $0.212697 \mathrm{E}-01$ $0.208656 \mathrm{E}-01$ $0.204699 \mathrm{E}-01$ $0.200822 E-01$ $0.19702 \mathrm{CE}-01$ $0.193293 \mathrm{E}-01$ $0.189638 \mathrm{E}-01$ $0.186061 \mathrm{E}-01$ $0.182558 \mathrm{E}-01$ $0.179123 \mathrm{E}-01$ $0.175757 \mathrm{E}-01$ $0.172458 E-01$ $0.168428 E-01$ $0.164499 \mathrm{E}-01$ $0.160675 E-01$ $0.156955 E-01$ $0.153331 \mathrm{E}-01$ $0.149796 \mathrm{E}-01$ $0.146351 \mathrm{E}-01$ $0.142993 \mathrm{E}-01$ $0.139718 \mathrm{E}-\mathrm{C}$ $0.136527 \mathrm{E}-01$ $0.133416 \mathrm{E}-01$ $0.130386 \mathrm{E}-\mathrm{O}$ $0.127433 E-01$ $0.124552 E-01$ $0.12174 \mathrm{CE}-01$ $0.118997 \mathrm{E}-01$ $0.116322 \mathrm{E}-01$ $0.113711 \mathrm{E}-01$ $0.111168 \mathrm{E}-01$ $0.108689 E-01$ $0.106275 \mathrm{E}-01$ $0.103922 \mathrm{E}-01$ $0.101628 \mathrm{E}-01$ $0.993967 \mathrm{E}-02$ $0.972178 E-02$ $0.950938 \mathrm{E}-02$ $0.93020 C E-02$ $0.909911 \mathrm{E}-02$ $0.890138 \mathrm{E}-02$ $0.870839 \mathrm{E}-02$ $0.851987 \mathrm{E}-02$ $0.833577 \mathrm{E}-02$ $0.815578 \mathrm{E}-02$ C. $798002 \mathrm{E}-02$ $0.780875 \mathrm{E}-02$ $0.764173 \mathrm{E}-02$ $0.747852 \mathrm{E}-02$
$0.55236 E-02$ $0.54207 \mathrm{E}-02$ $0.53226 \mathrm{E}-02$ $0.52271 \mathrm{E}-02$ $0.51343 E-02$ $0.5041 \mathrm{BE}-02$ $0.49512 E-02$ $0.48623 E-02$ $0.47746 \mathrm{E}-02$ $0.46887 \mathrm{E}-02$ $0.46046 \mathrm{E}-02$ $0.45225 E-02$ $0.44428 E-02$ $0.43636 \mathrm{E}-02$ $0.42866 E-02$ $0.42096 E-02$ $0.41345 E-02$ C. $40611 \mathrm{E}-02$ $0.39891 \mathrm{E}-02$ $0.39187 E-02$ $0.38486 E-02$ $0.37648 \mathrm{E}-02$ $0.36805 E-02$ $0.35956 \mathrm{E}-02$ $0.35145 E-02$ $0.34395 E-02$ $0.33657 \mathrm{E}-02$ $0.32931 \mathrm{E}-02$ $0.32224 \mathrm{E}-02$ $0.31530 \mathrm{E}-02$ $0.30851 E-02$ $0.30179 \mathrm{E}-02$ $0.29520 \mathrm{E}-02$ $0.28873 \mathrm{E}-02$ $0.28237 \mathrm{E}-02$ $0.27622 \mathrm{E}-02$ $0.27013 \mathrm{E}-02$ $0.26414 E-02$ $0.25834 \mathrm{E}-02$ $0.25270 E-02$ $0.24718 \mathrm{E}-02$ $0.24187 \mathrm{E}-02$ $0.23677 \mathrm{E}-02$ $0.23171 \mathrm{E}-02$ $0.22695 \mathrm{E}-02$ $0.22219 \mathrm{E}-02$ $0.21767 E-02$ $.21318 \mathrm{E}-02$ $0.20869 E-02$ $0.20438 E-02$ $0.20005 E-02$ $0.19584 \mathrm{E}-02$ $0.19179 \mathrm{E}-02$ $0.18768 \mathrm{E}-02$ $0.18369 \mathrm{E}-02$ $0.17981 \mathrm{E}-02$ $0.17604 E-02$
$.53513 E-04 \quad 0$. $.52590 E-04 \quad 0$. $0.51682 E-04 \quad 0$. $0.50790 E-04 \quad 0$. $0.49914 E-04 \quad 0$. $0.49052 E-04 \quad 0$ $0.48206 E-04 \quad 0$ $0.47374 E-04$ O. $0.45753 E-04$ $0.44963 E-04 \quad 0$ $0.44187 E-04 \quad 0$. $0.43424 E-04$ O. $0.42675 \mathrm{E}-04 \quad 0$. $0.41939 \mathrm{E}-04 \quad 0$. $0.41215 E-04 \quad 0$. $0.40503 E-04 \quad 0$. $0.39804 \mathrm{E}-04 \quad 0$ $0.39117 \mathrm{E}-04 \quad 0$. $0.39442 E-040$. $0.11707 \mathrm{E}-03 \quad 0$. $0.11505 E-03 \quad 0$. $0.11306 E-030$ $0.11111 E-03 \quad 0$. $0.10919 E-03 \quad 0$. $0.10731 \mathrm{E}-03 \quad 0$. $0.1 \cap 546 E-03 \quad 0$. $0.10364 \mathrm{E}-03 \quad 0$. $0.10185 \mathrm{E}-030$ $0.10009 \mathrm{E}-03 \quad 0$ $0.98362 \mathrm{E}-04 \quad 0$. $0.96664 \mathrm{E}-04 \quad 0$. $0.94996 E-04 \quad 0$. $0.93356 E-04 \quad 0$ $0.91745 t-04 \quad 0$ $0.90162 \mathrm{E}-040$ $0.88606 \mathrm{E}-040$. $0.87077 \mathrm{E}-04 \quad 0$ $0.85574 \mathrm{E}-04 \quad 0$ $0.84097 \mathrm{E}-04 \quad 0$. $0.82645 E-04 \quad 0$ $0.81219 E-04 \quad 0$ $0.79817 E-04 \quad 0$. $0.78440 \mathrm{E}-04 \quad 0$. $0.77086 E-040$ $0.75756 E-04 \quad 0$. $0.74448 E-04 \quad 0$. $0.73163 E-04 \quad 0$ $0.71901 \mathrm{E}-04 \quad 0$. $0.70660 \mathrm{E}-04 \quad 0$. $0.69440 E-04 \quad 0$ $0.68242 E-04 \quad 0$ $0.67064 E-04 \quad 0$ $0.65907 E-04 \quad 0$ $0.64769 E-04 \quad 0$. $0.63651 E-04$ O. $0.62553 E-04 \quad 0$ 


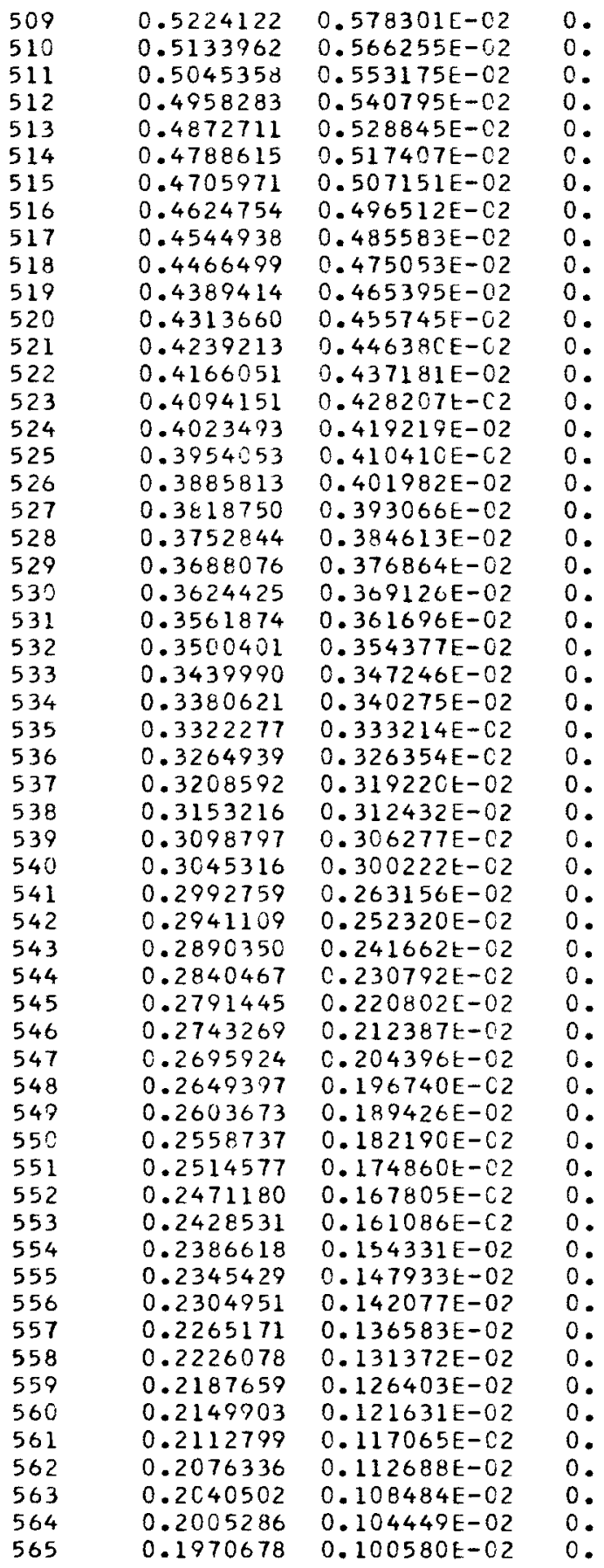

$0.181814 \mathrm{E} O 1 \quad 0.731898 \mathrm{E}-02$ $0.181860 \mathrm{t} 01$ $0.182068 \mathrm{t} \quad 01$ $0.182276 \mathrm{E} \quad 01$ $0.182438 \mathrm{E} 01$ $0.182600 E$ OI $0.182600 \mathrm{E} \quad 01$ $0.182646 \mathrm{E} \mathrm{OI}$ $0.182834 \mathrm{E}$ $0.182976 E$ OI $0.182976 \mathrm{E}$ OI 0.183021 E 01 $0.183067 \mathrm{E} 01$ C.183067t 01 $0.183067 \mathrm{E} 01$ $0.183113 t \quad 01$ $0.183159 E$ OI 0.183159 E 01 $0.183321 \mathrm{E} 01$ $0.183483 \mathrm{t}$ OI $0.183483 \mathrm{E} \quad 01$ $0.183529 \mathrm{E} C$ $0.183575 \mathrm{E}$ OI $0.183575 \mathrm{E}$ 01 $0.183575 \mathrm{E}$ OI $0.183575 \mathrm{E}$ OI $0.183621 \mathrm{E} \quad 01$ 0.183667 E 01 0.183829 E 01 $0.183991 \mathrm{t} \quad 01$ $0.183991 \mathrm{E} \quad 01$ $0.183991 \mathrm{E}$ OI $0.207491 \mathrm{E} \mathrm{O}$ $0.207861 \mathrm{E} 01$ C.208023E 01 $0.208023 t \quad 01$ $0.208185 E$ OI 0.208347 t O $0.208347 \mathrm{E}$ OI 0.208347 t 01 $0.208347 E$ OI 0.208347 E $0.208347 \mathrm{E} \mathrm{Cl}$ 0.208509 E 01 $0.208671 \mathrm{E} 01$ $0.208671 E$ OI 0.208753 E G1 $0.208915 E$ OI $0.208915 E$ E 0.208996 t OI $0.208996 \mathrm{~L} 01$ 0.208996 t 01 $0.208996 \mathrm{E} \quad 01$ C.208996t ?I $0.208996[\quad 0$ C. $208996 t 01$ $0.208996 t \quad 01$ $0.208996 E 01$

$0.142687 \mathrm{~F}-02$

$0.137297 E-02$
$0.17239 E-02$ $0.16884 \mathrm{E}-02$ C. $16529 \mathrm{E}-02$ $0.16184 \mathrm{E}-02$ $0.15853 E-02$ $0.15853 E-02$
$0.15529 E-02$ $0.15219 E-02$ $0.14906 E-02$ $0.14604 E-02$ $0.14321 E-02$ $0.14009 E-02$ $0.13724 E-02$ $0.13445 E-02$ $0.13167 \mathrm{E}-02$ $0.12895 E-02$ $0.12629 \mathrm{E}-02$ $0.12366 \mathrm{E}-02$ $0.12111 E-02$ $0.11861 E-02$ $0.11620 \mathrm{E}-02$ $0.11385 E-02$ $0.11155 E-02$ $0.10933 E-02$ $0.10711 E-02$ $0.10494 E-02$ $0.10282 \mathrm{E}-02$ $0.10073 \mathrm{E}-02$ $0.98679 E-03$ $0.96679 E-03$ $0.94734 E-03$ $0.92859 E-03$ $0.91014 E-03$ $0.89230 \mathrm{E}-03$ $0.85757 E-03$ $0.82162 E-03$ $0.78389 \mathrm{E}-03$ $0.75045 E-03$ $0.72216 E-03$ $0.69445 E-03$ C. $66792 \mathrm{E}-03$ $0.64258 \mathrm{E}-03$ $0.61751 \mathrm{E}-03$ $0.59306 \mathrm{E}-03$ C. $56925 E-03$ $0.54592 E-03$ $0.52287 \mathrm{E}-03$ $0.50135 E-03$ $0.48129 E-03$ $0.46220 \mathrm{E}-03$ C. $44412 \mathrm{E}-03$ $0.42688 \mathrm{E}-03$ $0.41033 E-03$ $0.39451 E-03$ $0.37935 E-03$ $0.36480 E-03$ $0.35084 \mathrm{~F}-03$ $0.33746 E-03$ $.60412 E-04$ $.57338 E-04$ $0.56349 E-04$ $0.55376 E-04$ $0.54421 E-04$ $0.53481 E-04$ $0.52558 E-04$ $0.51651 \mathrm{E}-04$ $0.50760 E-04$ $0.49884 E-04$ $0.49023 E-04$ $0.48177 \mathrm{E}-04$ $0.47345 E-04$ $0.46528 E-04$ $0.45725 E-04$ $0.44936 E-04$ $0.44161 E-04$ $0.43398 E-04$ $0.42649 E-04$ $0.41913 E-04$ $0.41190 E-04$ $0.40479 E-04$ $0.39780 E-04$ $0.39094 E-04$ $0.38419 E-04$ $0.37756 E-04$ $0.37105 \mathrm{E}-04$ $0.36464 E-04$ $0.35835 E-04$ $0.28709 E-04$ $0.28213 E-04$ $0.27726 \mathrm{E}-04$ $0.27248 E-04$ $0.26778 E-04$ $0.26315 E-04$ $0.25861 \mathrm{E}-04$ $0.25415 E-04$ $0.24976 E-04$ $0.24545 t-04$ $0.24122 \mathrm{E}-04$ $0.23705 \mathrm{E}-04$ $0.23296 E-04$ $0.22894 t-04$ $0.22499 E-04$ $0.22111 \mathrm{E}-04$ $0.21729 t-04$ $0.21354 E-04$ $0.20986 \mathrm{E}-04$ $0.20623 F-04$ $0.2 \backslash 268 E-04$ $0.19918 \mathrm{E}-04$ $0.19574 \mathrm{E}-04$ $0.13236 E-04$ $0.18904 E-04$

.18904E-04 0. 


$\begin{array}{lll}0.1936667 & 0.968735 \mathrm{E}-03 & 0 . \\ 0.1903243 & 0.933240 \mathrm{E}-03 & 0 . \\ 0.1870396 & 0.899282 \mathrm{E}-03 & 0 . \\ 0.1838116 & 0.866838 \mathrm{E}-03 & 0 . \\ 0.1806393 & 0.835820 \mathrm{E}-03 & 0 . \\ 0.1775217 & 0.806137 \mathrm{E}-03 & 0 . \\ 0.1744580 & 0.777715 \mathrm{E}-03 & 0 . \\ 0.1714471 & 0.750489 \mathrm{E}-03 & 0 . \\ 0.1684882 & 0.724397 \mathrm{E}-03 & 0 . \\ 0.1655804 & 0.699387 \mathrm{E}-03 & 0 . \\ 0.1627227 & 0.675406 \mathrm{E}-03 & 0 . \\ 0.1599144 & 0.652410 \mathrm{E}-03 & 0 . \\ 0.1571545 & 0.630357 \mathrm{E}-03 & 0 . \\ 0.1544423 & 0.609205 \mathrm{E}-03 & 0 . \\ 0.1517768 & 0.588915 \mathrm{E}-03 & 0 . \\ 0.1491574 & 0.569451 \mathrm{E}-03 & 0 . \\ 0.1465832 & 0.550775 \mathrm{E}-03 & 0 . \\ 0.1440534 & 0.532853 \mathrm{E}-03 & 0 . \\ 0.1415672 & 0.515648 \mathrm{E}-03 & 0 . \\ 0.1391240 & 0.499129 \mathrm{E}-03 & 0 . \\ 0.1367230 & 0.483265 \mathrm{E}-03 & 0 . \\ 0.1343633 & 0.468026 \mathrm{E}-03 & 0 . \\ 0.1320444 & 0.453384 \mathrm{E}-03 & 0 . \\ 0.1297655 & 0.439312 \mathrm{E}-03 & 0 . \\ 0.1275260 & 0.425786 \mathrm{E}-03 & 0 . \\ 0.1253251 & 0.412781 \mathrm{E}-03 & 0 . \\ 0.1231622 & 0.400274 \mathrm{E}-03 & 0 . \\ 0.1210366 & 0.388244 \mathrm{E}-03 & 0 . \\ 0.1189477 & 0.376670 \mathrm{E}-03 & 0 . \\ 0.1168948 & 0.365532 \mathrm{E}-03 & 0 . \\ 0.1148774 & 0.354811 \mathrm{E}-03 & 0 . \\ 0.1128948 & 0.344489 \mathrm{E}-03 & 0 . \\ 0.1109464 & 0.334549 \mathrm{E}-03 & 0 . \\ 0.1090317 & 0.324974 \mathrm{E}-03 & 0 . \\ 0.1071500 & 0.315748 \mathrm{E}-03 & 0 . \\ 0.1053007 & 0.306857 \mathrm{E}-03 & 0 . \\ 0.1034834 & 0.298286 \mathrm{E}-03 & 0 . \\ 0.1016974 & 0.290022 \mathrm{E}-03 & 0 . \\ 0.0999423 & 0.282051 \mathrm{E}-03 & 0 .\end{array}$

$0.208996 E$ OI $0.208996 E$ OI $0.208996 \mathrm{t} 01$ 0.208996 E 01 0.208996 E 01 $0.208996 \mathrm{E}$ OI $0.208996 \mathrm{E} 01$ $0.208996 \mathrm{E} 01$ $0.208996 E$ O1 $0.208996 \mathrm{E}$ O1 $0.208996 \mathrm{E}$ OI $0.208996 E$ OI $0.208996 E$ O1 0.208996 E 01 $0.208996 \mathrm{E} 01$ $0.208996 \mathrm{E} O 1$ 0.208996 E OI $\begin{array}{lll}0.208996 E & 01 \\ 0.208996 E & 01\end{array}$ $0.208996 \mathrm{E} \quad 01$ $0.208996 \mathrm{E} 01$ $0.208996 E$ O1 $0.208996 \mathrm{E}$ OI $0.208996 \mathrm{E} 01$ $0.208996 E \quad 01$ $0.208996 \mathrm{t} 01$ $0.208996 \mathrm{E} \quad 01$ $0.208996 \mathrm{E} 01$ $0.208996 \mathrm{E}$ OI $0.208996 \mathrm{E}$ 0I $0.208996 \mathrm{E} 01$ $0.208996 \mathrm{E} \quad 01$ 0.208996 E 01 $0.208996 \mathrm{E}$ OI $0.208996 t \quad 01$ 0.208996 E 01 $0.208996 \mathrm{E} \quad 01$ $0.208996 E \quad 01$ $0.208996 \mathrm{E} 01$ 0.208996 E OI
$0.132142 E-02$ $0.127213 E-02$ $0.122497 t-02$ $0.113669 E-02$ $0.109536 \mathrm{E}-02$ $0.105580 \mathrm{E}-02$ $0.101792 E-02$ $0.981650 E-03$ $0.946908 E-03$ $0.913628 \mathrm{E}-03$ $0.881743 E-03$ $0.851190 \mathrm{E}-03$ $0.821907 E-03$ $0.793838 \mathrm{E}-03$ C. $766926 E-03$ $0.741119 \mathrm{E}-03$ $0.716366 \mathrm{E}-03$ $0.692621 E-03$ $0.669837 E-03$ $0.647971 \mathrm{E}-03$ $0.626982 \mathrm{E}-03$ $0.606830 \mathrm{E}-03$ $0.587478 E-03$ $0.56889 \mathrm{CE}-03$ $0.551033 E-03$ $0.533874 \mathrm{E}-03$ $0.517381 \mathrm{E}-03$ $0.501526 E-03$ $0.486280 E-03$ $0.471616 E-03$ $0.457510 \mathrm{E}-03$ $0.443936 E-03$ $0.430872 \mathrm{E}-03$ C. $418295 \mathrm{E}-03$ $0.406184 \mathrm{E}-03$ $0.39451 \mathrm{GE}-03$ $0.383281 E-03$ $0.372452 \mathrm{E}-03$
$0.32466 E-03$ $0.31240 E-03$ $0.30069 E-03$ $0.28950 E-03$ $0.27881 E-03$ $0.26860 E-03$ $0.25882 \mathrm{E}-03$ $0.24946 E-03$ $0.24050 E-03$ $0.23192 E-03$ $0.22370 E-03$ $0.21582 E-03$ $0.20827 E-03$ $0.20103 E-03$ $0.19410 E-03$ $0.18746 E-03$ $0.18109 \mathrm{E}-03$ $0.17498 E-03$ $0.16912 E-03$ $0.16350 \mathrm{E}-03$ $0.15811 E-03$ $0.15294 E-03$ $0.14797 \mathrm{E}-03$ $0.14321 E-03$ $0.13863 E-03$ $0.13423 E-0.3$ $0.13001 \mathrm{E}-03$ $0.12595 E-03$ $0.12205 E-03$ $0.1183 \mathrm{CE}-03$ $0.11469 E-03$ $0.11123 E-03$ $0.10789 E-03$ $0.10468 E-03$ C. $10160 E-03$ $0.98622 \mathrm{E}-04$ $0.95760 E-04$ $0.93003 E-04$ C. $90347 \mathrm{E}-04$
$0.18578 \mathrm{E}-04 \quad 0$. $0.18257 E-04 \quad 0$. $0.17942 \mathrm{E}-04 \quad 0$. $0.17633 \mathrm{E}-04 \quad 0$. $0.17328 \mathrm{E}-04 \quad 0$. $0.17029 \mathrm{E}-040$. $0.16735 E-04 \quad 0$. $0.16446 \mathrm{E}-04 \quad 0$. $0.16163 E-040$. $0.15884 \mathrm{E}-04 \quad 0$. $0.15610 E-040$. $0.15340 \mathrm{E}-04 \quad 0$. $0.15075 E-04 \quad 0$. $0.14815 \mathrm{E}-040$. $0.14560 \mathrm{E}-04 \quad 0$. $0.14308 E-04 \quad 0$. $0.14061 \mathrm{E}-04 \quad 0$. $0.13819 \mathrm{E}-04 \quad 0$. $0.13580 E-04 \quad 0$. $0.13346 \mathrm{E}-04 \quad 0$. $0.13115 \mathrm{E}-04 \quad 0$. $0.12889 \mathrm{E}-04 \quad 0$. $0.12667 \mathrm{E}-04 \quad 0$. $0.12448 E-04 \quad 0$. $0.12233 E-040$. $0.12022 \mathrm{E}-04 \quad 0$. $0.11815 \mathrm{E}-04 \quad 0$. $0.11611 \mathrm{E}-040$. $0.11410 E-040$. $0.11213 \mathrm{E}-04 \quad 0$. $0.11020 \mathrm{E}-040$. 0.10830 E-04 0. $0.10643 E-04 \quad 0$. $0.10459 E-040$. $0.10279 E-040$. $0.10101 \mathrm{E}-04 \quad 0$. $0.99269 E-050$. $0.97556 E-05$ O $0.95872 \mathrm{E}-05 \quad 0$. 
hCBC equivalent

BUCKLING $=0.002406$

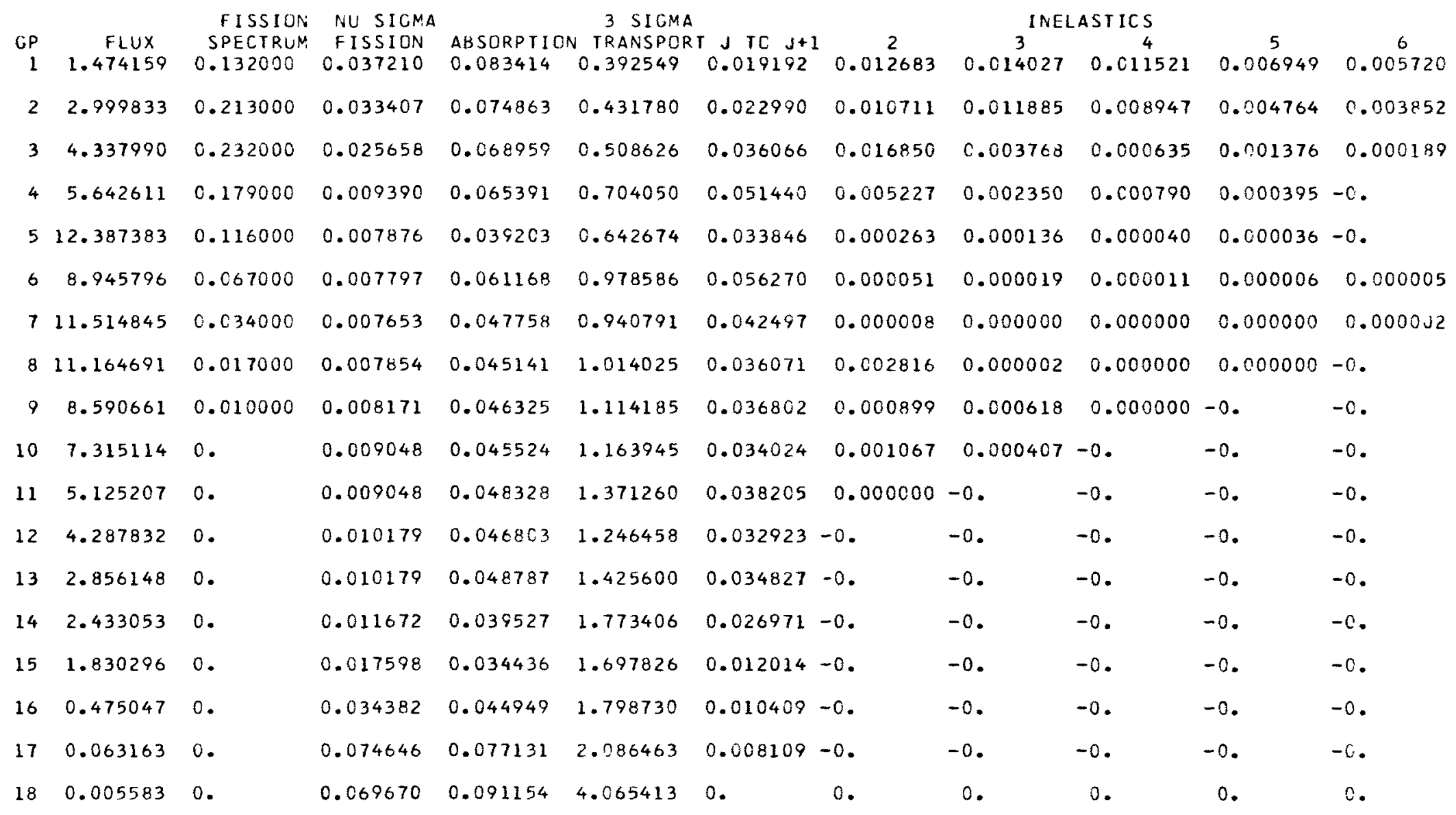


RE 196 ELMOE

S A M P LE PROBLEMNO. 2

$\begin{array}{cccc}\text { MATERIAL } & \text { AT.WT } & \text { ATOMS/CC(E-24) } & \text { VOL.FRAC. } \\ & & & \\ 1 & 12.011000 & 1.00000000 & -0 . \\ 2 & 16.000000 & 1.00000000 & 0.03120000 \\ 3 & 22.991000 & 1.00000000 & 0.00330000 \\ 4 & 56.460000 & 1.00000000 & 0.01525000 \\ 5 & 238.000000 & 1.00000000 & -0 . \\ 6 & 239.000000 & 1.00000000 & -0 . \\ 7 & 238.000000 & 1.00000000 & 0.01403999 \\ 8 & 239.000000 & 1.00000000 & 0.00156000 \\ 9 & 238.000000 & 1.00000000 & -0 . \\ 10 & 239.000000 & 1.00000000 & -0 .\end{array}$

\section{MATERIAL CONSTANTS}

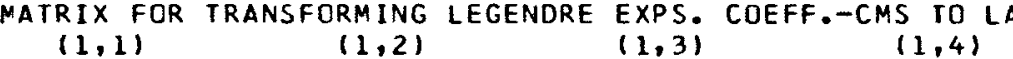$$
(1,2)
$$$$
(1,3)
$$$$
(1,4)
$$

$0.99583 E 00$ $0.99765 \mathrm{E} \mathrm{OO}$ $0.99886 E \quad 00$ $-0.33149 E-01$ $-0.24927 \mathrm{E}-01$ $-0.17371 \mathrm{E}-01$

$0.17588 E-02$ $0.98550 E-03$ $0.46996 \mathrm{E}-03$ $0.87558 E-04$

$-0.11722 E-03$ $-0.52205 E-04$ $-0.19865 E-04$ $0.99975 E$ OO $-0.80007 E-02$

$-0.34335 E-05$

DPTION 1

CALCULATED RIAL ALPHA

$10.2816316 \mathrm{E}-00$

$20.2162987 \mathrm{E}-00$

$3 \quad 0.1597795 E-00$

$4 \quad 0.6726708 \mathrm{E}-01$ 
GROUP

$\begin{aligned} 2 & -0 . \\ 3 & -0 . \\ 4 & -0 . \\ 5 & -0 . \\ 6 & -0 . \\ 7 & -0 . \\ 8 & -0 . \\ 9 & -0 . \\ 10 & -0 . \\ 11 & -0 . \\ 12 & -0 . \\ 13 & -0 . \\ 14 & -0 . \\ 15 & -0 . \\ 16 & -0 . \\ 17 & -0 .\end{aligned}$

$$
2
$$

$0.7829 E-02 \quad 0.1056 E-02 \quad 0.1501 E-02$

$0.1759 \mathrm{E}-01 \quad 0.9204 \mathrm{E}-03 \quad 0.1558 \mathrm{E}-02$

$0.3532 E-01 \quad 0.2039 E-02 \quad 0.3405 E-02$

$0.2115 E-01 \quad 0.1652 E-02 \quad$ C. $2705 E-02$

$0.4344 \mathrm{E}-01 \quad 0.2417 \mathrm{E}-02 \quad 0.2512 \mathrm{E}-02$

$0.2776 E-01 \quad 0.2569 E-02 \quad C .3432 E-02$

$0.2521 E-010.1766 E-02 \quad C .3556 E-02$

$0.2495 E-01 \quad 0.2142 E-02 \quad C .3463 E-02$

$0.2492 E-01 \quad 0.2022 E-02 \quad 0.4773 E-02$

$0.2782 E-01 \quad 0.2907 E-02 \quad 0.4896 E-02$

$0.2019 E-01 \quad 0.1895 E-02 \quad C .8288 E-02$

$0.2263 E-01 \quad 0.2743 E-02 \quad 0.6785 E-02$

$0.1374 E-01 \quad 0.4817 E-02 \quad 0.5635 E-02$

$0.6497 \mathrm{E}-02 \quad 0.3957 \mathrm{E}-03 \quad 0.2234 \mathrm{E}-02$

$0.5218 E-02 \quad 0.3170 E-03 \quad$ C. $2126 E-C 2$

$0.3792 E-02 \quad 0.2279 E-03 \quad 0.1557 E-02$

BUCKLING

INITIAL VALUE $0.2000000 E-02$

FINAL VALUE 0.2362230 E-02

COARSE GROUP DATA

FINE GROUP

\begin{tabular}{|c|c|c|c|c|}
\hline GROUP & CORRESPCNDENCE & FLUX & CURRENT/B & $1 / 0$ \\
\hline 1 & $0-0$ & $0.147599 \mathrm{E} \quad 01$ & 0. & $0.39255 \mathrm{E}-00$ \\
\hline 2 & $1-29$ & $0.300395 \mathrm{E} 01$ & 0. & $0.43176 E-00$ \\
\hline 3 & $30-58$ & $0.434479 E \quad 01$ & 0. & $0.50862 \mathrm{E}$ \\
\hline 4 & $59-86$ & $0.565207 E \quad 01$ & 0. & $0.70404 \mathrm{E}$ \\
\hline 5 & $87-115$ & $0.124196 E \quad 02$ & 0. & $0.64267 E$ \\
\hline 6 & $116-144$ & $0.897294 \mathrm{E} \quad 01$ & 0. & $0.97857 \mathrm{E}$ \\
\hline 7 & $145-173$ & $0.115559 \mathrm{E} \quad 02$ & 0 . & $0.94079 E$ \\
\hline 8 & $174-202$ & $0.112124 E \quad 02$ & 0. & $0.10140 \mathrm{E}$ \\
\hline 9 & $203-230$ & $0.863374 E \quad 01$ & 0. & $0.11142 \mathrm{E}$ \\
\hline 10 & $231-259$ & $0.735728 E \quad 01$ & 0. & $0.11639 \mathrm{E}$ \\
\hline 11 & $260-287$ & $0.515829 E \quad 01$ & 0. & $0.13713 E$ \\
\hline 12 & $288-316$ & $0.431398 \mathrm{E} \quad 01$ & 0. & $0.12464 \mathrm{E}$ \\
\hline 13 & $317-345$ & $0.287096 \mathrm{E} \mathrm{OI}$ & 0. & $0.14256 E$ \\
\hline 14 & $346-392$ & $0.243074 \mathrm{E} \quad 01$ & 0. & $0.17735 \mathrm{E}$ \\
\hline 15 & $393-471$ & $0.177067 \mathrm{E} \quad 01$ & 0. & $0.17010 \mathrm{E}$ \\
\hline 16 & $472-540$ & $0.424069 E-00$ & 0. & $0.17981 \mathrm{E}$ \\
\hline 17 & $541-604$ & $0.502290 \mathrm{E}-01$ & 0. & $0.20863 \mathrm{E}$ \\
\hline 18 & & $0.391853 E-02$ & 0. & $0.40654 \mathrm{E}$ \\
\hline
\end{tabular}

MATERIAL 
FINE CROUP CATA

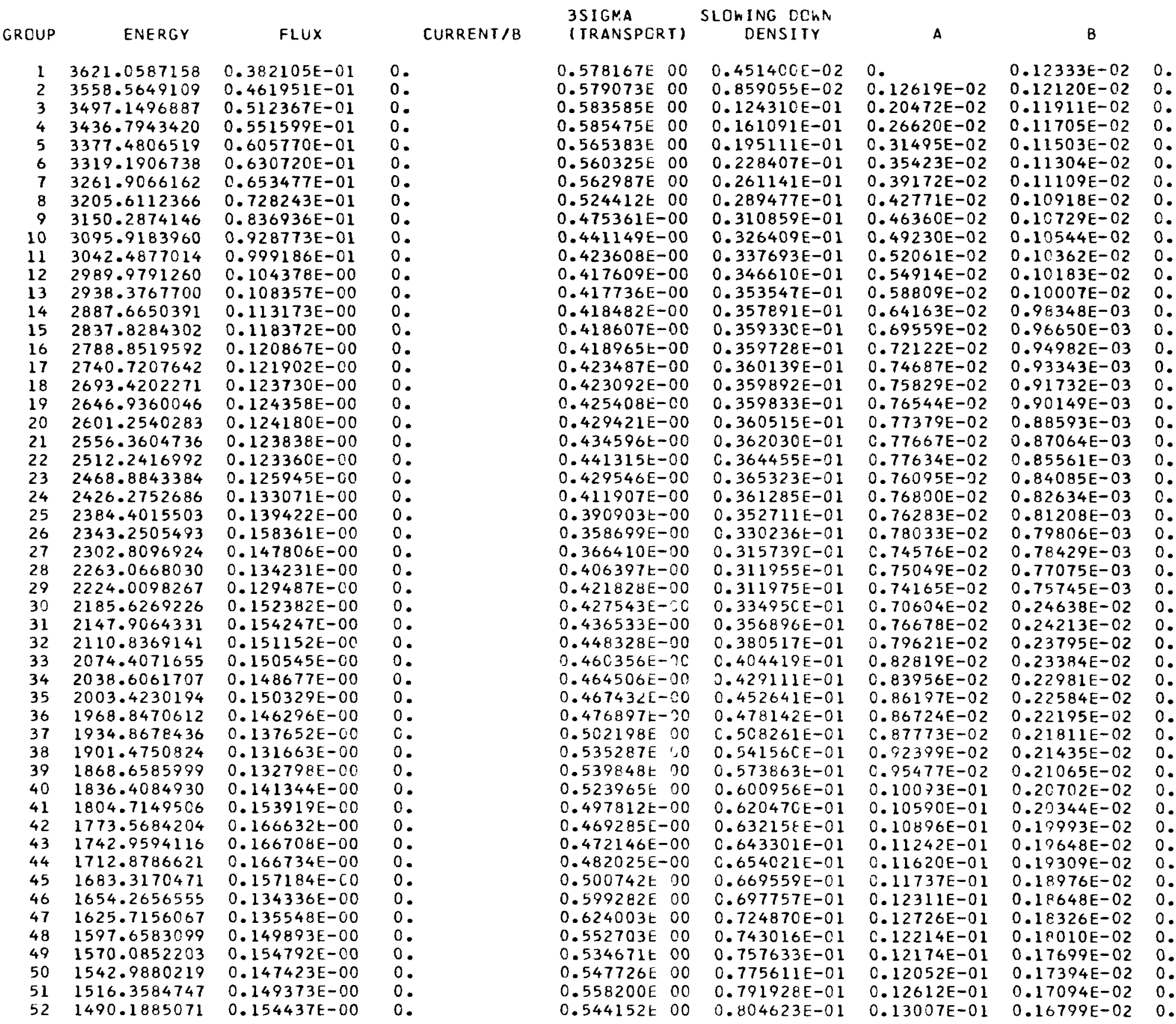




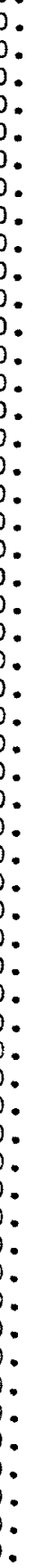

$0.550596 E$ OO $0.573963 E \quad 00$ $0.571312 \mathrm{E} \mathrm{OO}$ $0.548014 E$ OO $0.514728 \mathrm{E} \quad 00$ $0.497405 \mathrm{E}-00$ $0.592429 \mathrm{E} 00$ $0.733206 E$ OO $0.739039 \mathrm{E} 00$ $0.677383 \mathrm{E} \quad 00$ $0.643746 E \quad 00$ $0.622764 \mathrm{E} \quad 00$ $0.611033 \mathrm{t} 00$ $0.617106 \mathrm{E} \mathrm{OO}$ $0.683196 \mathrm{E} \mathrm{GO}$ $0.688828 \mathrm{E} \quad 00$ $0.653334 \mathrm{E} \quad 00$ $0.696769 \mathrm{E} 00$ $0.729429 E \quad 00$ $0.760902 \mathrm{E} 00$ $0.881805 E 00$ $0.104099 \mathrm{E} 01$ $0.107594 \mathrm{E} 01$ $0.102161 \mathrm{E} 01$ $0.972137 \mathrm{E} 00$ $0.914432 \mathrm{E} 00$ $0.822282 \mathrm{E} \quad 00$ $0.739510 \mathrm{E}$ OO $0.702524 E$ OO $0.678386 \mathrm{t} \quad 00$ 0.652287 E 00 $0.642509 \mathrm{E} 00$ $0.639438 \mathrm{E} C \mathrm{CO}$ $0.635051 \mathrm{E} \quad 00$ $0.647413 E$ OO $0.653574 \mathrm{E} \quad 00$ $0.667403 \mathrm{E} \quad 00$ $0.679891 E 00$ $0.679220 \mathrm{E} 00$ $0.661661 E 00$ 0.649880 E OO $0.653434 E \quad 00$ $0.658563 E \quad 00$ $0.659002 \mathrm{E} \quad 00$ $0.657840 E$ OO $0.653190 E$ OO 0.646517 t 00 $0.640590 \mathrm{E} \quad 00$ $0.635620 \mathrm{t}$ OO $0.631819 E$ OO $\begin{array}{ll}0.631819 E & 00 \\ 0.629593 t & 00\end{array}$ $0.629593 \mathrm{E} 00$
$0.630407 \mathrm{E}$ $0.633423 \mathrm{E} 00$ 0.637090 C 00 $0.635485 E$ OO $0.635485 E$
$0.632586 E$ $0.632586 \mathrm{E}$
0.60
$0.632623 \mathrm{E}$
$818973 E-01$ $.834146 \mathrm{E}-01$ $0.847803 \mathrm{E}-01$ $0.857969 E-01$ $0.862612 \mathrm{E}-01$ $0.871958 \mathrm{E}-01$ $0.954472 E-01$ $0.103791 \mathrm{E}-00$ $0.111344 \mathrm{E}-00$ $0.117708 \mathrm{E}-00$ $0.123212 \mathrm{E}-00$ $0.128282 \mathrm{E}-00$ $0.133278 \mathrm{E}-00$ $0.138149 E-00$ $0.143840 \mathrm{E}-00$ $0.149459 \mathrm{E}-00$ $0.154571 E-00$ $0.160091 \mathrm{E}-00$ $0.165773 \mathrm{E}-00$ $0.171452 \mathrm{E}-00$ $0.177665 \mathrm{E}-00$ $0.184469 \mathrm{E}-00$ C. $191339 \mathrm{E}-00$ $0.197899 \mathrm{E}-00$ $0.204023 E-00$ $0.209703 \mathrm{E}-00$ $0.214697 \mathrm{E}-00$ $0.218950 \mathrm{E}-00$ $0.222578 \mathrm{E}-00$ $0.225690 \mathrm{E}-00$ $0.228050 \mathrm{E}-00$ C. $22950 \mathrm{CE}-00$ $0.230208 \mathrm{E}-00$ $0.23038 \mathrm{CE}-00$ $0.237031 \mathrm{E}-00$ $0.24336 C E-0 O$ $0.249517 \mathrm{E}-00$ $0.255459 \mathrm{E}-00$ $0.260960 E-00$ $0.265905 \mathrm{E}-00$ C. $270501 E-00$ $0.274945 \mathrm{E}-00$ $0.279208 E-00$ $0.283219 \mathrm{E}-00$ $0.286943 \mathrm{E}-00$ $0.290321 \mathrm{E}-00$ $0.293386 \mathrm{E}-00$ $0.296188 \mathrm{E}-00$ $0.298739 \mathrm{E}-00$ $0.301009 E-00$ $0.303054 \mathrm{E}-00$ C. $304904 \mathrm{E}-00$ $0.306599 E-00$ $0.308172 E-00$ $0.309585 E-00$ $0.310926 E-00$ C. 31225 CE-OO
$13172 E-01$ $0.14279 E-01$ $0.14768 \mathrm{E}-01$ $0.15733 \mathrm{E}-01$ $0.16720 E-01$ $0.18145 \mathrm{E}-01$ $0.21471 E-01$ $0.22033 \mathrm{E}-01$ $0.22084 \mathrm{E}-01$ $0.22668 \mathrm{E}-01$ $0.22597 E-01$ $0.21935 E-01$ $0.22572 \mathrm{E}-01$ $0.22728 E-01$ $0.22368 \mathrm{E}-01$ $0.22504 E-01$ $0.22664 \mathrm{E}-01$ $0.23089 E-01$ $0.24279 \mathrm{E}-01$ $0.26687 \mathrm{E}-01$ C. 28077E-01 C. $28425 \mathrm{E}-01$ C. $28408 \mathrm{E}-01$ $0.28220 \mathrm{E}-01$ $0.27403 E-01$ $0.26983 \mathrm{E}-01$ $0.27083 E-01$ $0.28231 \mathrm{E}-01$ $0.29021 \mathrm{E}-01$ $0.31187 \mathrm{E}-01$ $0.34879 \mathrm{E}-01$ $0.38307 E-01$ $0.40499 E-01$ $0.43410 E-01$ $0.45995 \mathrm{E}-01$ $0.47773 E-01$ $0.49844 \mathrm{E}-01$ C. $51747 E-01$ $0.52636 \mathrm{E}-01$ $0.53004 E-01$ $0.53784 E-01$ $0.54717 \mathrm{E}-01$ $0.55492 E-01$ $0.56115 E-01$ $0.56832 \mathrm{E}-01$ $0.57000 E-01$ $0.57117 \mathrm{E}-01$ $0.57396 E-01$ $0.58164 E-01$ $0.59150 E-01$ $0.60439 E-01$ $0.61598 E-01$ $0.62567 E-01$ $0.63290 E-01$ $0.63554 \mathrm{E}-01$ $0.64148 E-01$
$.16509 E-02 \quad 0$. $.16224 \mathrm{E}-020$. $.15944 \mathrm{E}-020$. $0.15669 \mathrm{E}-02$ O. $0.15398 \mathrm{E}-02 \quad 0$. $0.15132 \mathrm{E}-02 \quad 0$. $0.54742 E-02 \quad 0$ $0.53798 E-02$ O. $0.52869 \mathrm{E}-02$ O. $0.51957 E-02 \quad 0$ $0.51060 \mathrm{E}-02$ 0 $0.50179 E-02 \quad 0$. $0.47313 E-02$. $0.48462 E-02$ $0.47625 E-02 \quad 0$ $0.46803 E-02 \quad 0$. $0.45996 \mathrm{E}-02$. $0.45202 E-02$ O. $0.44422 \mathrm{E}-02$ $0.43655 E-02 \quad 0$. $0.42902 \mathrm{E}-02 \quad 0$. $0.42161 \mathrm{E}-02$ 0 . $0.41434 E-02$ O. $0.4 C 718 E-02 \quad 0$ $0.40016 \mathrm{E}-02 \quad 0$. $0.39325 \mathrm{E}-02 \quad 0$ $0.38646 \mathrm{E}-02$ 0. $0.37979 E-02$ O $0.37324 \mathrm{E}-02$ 0. $0.36680 \mathrm{E}-02 \quad 0$. $0.36047 \mathrm{E}-02 \quad 0$ $0.35425 \mathrm{E}-02 \quad 0$. $0.34813 \mathrm{E}-02$ 0 . $0.34212 \mathrm{E}-02$ 0. $0.81106 \mathrm{E}-02 \quad 0$ $0.79706 \mathrm{E}-02 \quad 0$ $0.78330 \mathrm{E}-02 \quad 0$. $0.76978 \mathrm{E}-02 \quad 0$. $0.75650 \mathrm{E}-02 \quad 0$. $0.74344 \mathrm{E}-02 \quad 0$ $0.73061 \mathrm{E}-02 \quad 0$. $0.71800 \mathrm{E}-02 \quad 0$. $0.70561 \mathrm{E}-02 \quad 0$. $0.69343 E-02$. $0.68147 \mathrm{E}-02$ 0. $0.66970 E-02 \quad 0$. $0.65815 E-02 \quad 0$. $0.64679 \mathrm{E}-02 \quad 0$. $0.63563 \mathrm{E}-02$ 0 $0.62466 E-02 \quad 0$ $0.61387 \mathrm{E}-02 \quad 0$. $0.60328 \mathrm{E}-020$. $0.59287 \mathrm{E}-020$. $0.5 \times 264 t-020$. $0.57258 E-02 \quad 0$. $0.56270 E-02 \quad 0$. $0.55299 E-02 \quad 0$. 


$\begin{array}{llll}110 & 542.9028625 & 0.434235 \mathrm{E}-00 & 0 . \\ 111 & 533.5332031 & 0.434794 \mathrm{E}-00 & 0 . \\ 112 & 524.3252563 & 0.440430 \mathrm{E}-00 & 0 . \\ 113 & 515.2762222 & 0.443880 \mathrm{E}-00 & 0 . \\ 114 & 506.3833733 & 0.442285 \mathrm{E}-00 & 0 . \\ 115 & 497.6439857 & 0.437360 \mathrm{E}-00 & 0 . \\ 116 & 489.0554314 & 0.441929 \mathrm{E}-00 & 0 . \\ 117 & 480.6151085 & 0.413213 \mathrm{E}-00 & 0 . \\ 118 & 472.3204422 & 0.358752 \mathrm{E}-00 & 0 . \\ 119 & 464.1689262 & 0.298592 \mathrm{E}-00 & 0 . \\ 120 & 456.1581039 & 0.244285 \mathrm{E}-00 & 0 . \\ 121 & 448.2855225 & 0.199591 \mathrm{E}-00 & 0 . \\ 122 & 440.5488243 & 0.181884 \mathrm{E}-00 & 0 . \\ 123 & 432.9456444 & 0.175341 \mathrm{E}-00 & 0 . \\ 124 & 425.4736786 & 0.180821 \mathrm{E}-00 & 0 . \\ 125 & 418.1306686 & 0.204198 \mathrm{E}-00 & 0 . \\ 126 & 410.9143906 & 0.224456 \mathrm{E}-00 & 0 . \\ 127 & 403.8226547 & 0.230485 \mathrm{E}-00 & 0 . \\ 128 & 396.8533058 & 0.242866 \mathrm{E}-00 & 0 . \\ 129 & 390.0042381 & 0.252644 \mathrm{E}-00 & 0 . \\ 130 & 383.2733803 & 0.266539 \mathrm{E}-00 & 0 . \\ 131 & 376.6586838 & 0.291203 \mathrm{E}-00 & 0 . \\ 132 & 370.1581421 & 0.316953 \mathrm{E}-00 & 0 . \\ 133 & 363.7697945 & 0.315570 \mathrm{E}-00 & 0 . \\ 134 & 357.4916992 & 0.329277 \mathrm{E}-00 & 0 . \\ 135 & 351.3219528 & 0.364017 \mathrm{E}-00 & 0 . \\ 163 & 215.7776318 & 0.429485 \mathrm{E}-00 & 0 . \\ 164 & 212.0536461 & 0.431787 \mathrm{E}-00 & 0 . \\ 165 & 208.3939323 & 0.411423 \mathrm{E}-00 & 0 . \\ 166 & 204.7973824 & 0.382843 \mathrm{E}-00 & 0 . \\ 136 & 345.2586861 & 0.344702 \mathrm{E}-00 & 0 . \\ 137 & 339.3000641 & 0.342468 \mathrm{E}-00 & 0 . \\ 138 & 333.4442787 & 0.359021 \mathrm{E}-00 & 0 . \\ 139 & 327.6895561 & 0.343286 \mathrm{E}-00 & 0 . \\ 139 & 227.31 & & \end{array}$

$0.632724 t 00$ $0.630652 \mathrm{E} 0 \mathrm{O}$ $0.623568 \mathrm{E} 00$ $0.620465 \mathrm{E} 00$ 0.625259 E 00 $0.629305 \mathrm{E} \mathrm{OO}$ 0.728101100 $0.778910 \mathrm{E} 00$ $0.870464 \mathrm{E} 00$ $0.101218 E 01$ $0.118725 \mathrm{t}$ 01 $0.142574 \mathrm{E}$ ol 0.158786 E OI $0.165908 \mathrm{E} 01$ $0.162203 E 01$ $0.143882 \mathrm{E}$ O1 $0.130573 \mathrm{E} 01$ $0.128331 \mathrm{E} 01$ $0.124158 \mathrm{E} 01$ $0.117964 \mathrm{E} \mathrm{OI}$ $0.112364 \mathrm{E} 01$ $0.104914 \mathrm{E}$ OI $0.990107 E 00$ 0.983768 E 00 $0.969852 E$ OO $0.918065 \mathrm{E} 00$ $0.954132 \mathrm{E} 00$ $0.953038 \mathrm{E} 00$ $0.900474 E 00$ $0.924634 \mathrm{E} 00$ 0.936889 E 00 0.924169 E 00 $0.871955 \mathrm{E} 00$ 0.824467 E 00 $0.819912 \mathrm{E} 00$ $0.900632 \mathrm{E} 00$ 0.912533 E 00 $0.925669 \mathrm{E} 00$ $0.936920 \mathrm{E} \mathrm{OO}$ 0.966229 E 00 $0.973042 \mathrm{E} \mathrm{OO}$ 0.913050 E OO $0.884426 E 00$ $0.910831 \mathrm{E} 00$ $0.930947 \mathrm{E} \mathrm{OO}$ $0.943843 \mathrm{E} 00$ 0.962691 E 00 $0.964461 \mathrm{E} \mathrm{OO}$ $0.943787 E 00$ $0.947204 E$ DO 0.952505 E 00 $0.945988 \mathrm{E} \quad 00$ 0.933445 E 00 $0.904231 \mathrm{E} 0 \mathrm{O}$ 0.889755 t 00 0.901539 E 00 $0.926095 \mathrm{E} 00$
$0.313487 E-00$ $0.314552 \mathrm{E}-00$ $0.315345 E-00$ $0.315918 E-00$ $0.316381 E-00$ $0.316793 E-00$ $0.320865 E-00$ $0.325286 \mathrm{E}-00$ $0.330487 \mathrm{E}-00$ $0.336543 E-00$ $0.343315 \mathrm{E}-00$ $0.350629 \mathrm{E}-00$ $0.358045 E-00$ $0.365386 \mathrm{E}-00$ $0.372466 E-00$ $0.379000 \mathrm{E}-00$ $0.385032 \mathrm{E}-00$ $0.390800 \mathrm{E}-00$ $0.396206 \mathrm{E}-00$ $0.401281 E-00$ $0.405962 E-00$ $0.410076 E-00$ $0.413604 E-00$ $0.416996 \mathrm{E}-00$ $0.420019 \mathrm{E}-00$ $0.422316 E-00$ $0.424799 E-00$ $0.427173 E-00$ $0.429103 \mathrm{E}-00$ $0.431162 E-00$ $0.432965 E-00$ $0.434242 \mathrm{E}-00$ $0.434595 \mathrm{E}-0 \mathrm{O}$ $0.434283 \mathrm{E}-00$ $0.434011 E-00$ $0.433491 E-00$ $0.433221 \mathrm{E}-00$ $0.432935 \mathrm{E}-00$ $0.432511 \mathrm{E}-00$ C. $432210 \mathrm{E}-00$ $0.431612 \mathrm{E}-00$ $0.430029 \mathrm{E}-00$ $0.428155 E-00$ $0.426737 \mathrm{E}-00$ $0.425635 \mathrm{E}-00$ $0.424635 E-00$ $0.423616 \mathrm{E}-00$ $0.422376 E-00$ $0.420759 \mathrm{E}-00$ $0.419159 E-00$ $0.417521 \mathrm{E}-00$ $0.415776 \mathrm{E}-00$ $0.413778 \mathrm{E}-00$ $0.411288 \mathrm{E}-00$ $0.408654 E-00$ $0.406289 E-00$ $0.404343 \mathrm{E}-00$
$0.65431 E-01$ $0.67107 E-01$ $0.69254 E-01$ $0.71712 \mathrm{E}-\mathrm{O} 1$ $0.74412 \mathrm{E}-01$ $0.77236 E-01$ $0.80121 \mathrm{E}-01$ $0.82769 E-01$ $0.84771 E-01$ $0.86343 \mathrm{E}-01$ $0.86804 \mathrm{E}-01$ $0.85728 \mathrm{E}-01$ $0.83280 \mathrm{E}-01$ $0.79764 \mathrm{E}-01$ $0.75566 \mathrm{E}-01$ $0.70881 E-01$ $0.66532 \mathrm{E}-01$ $0.62565 E-01$ $0.59271 E-01$ C. $56570 \mathrm{E}-01$ C. $55711 \mathrm{E}-01$ $0.54952 E-01$ $0.55267 E-01$ $0.55271 \mathrm{E}-01$ $0.56177 \mathrm{E}-01$ $0.57255 E-01$ $0.57462 E-01$ $0.57502 E-01$ $0.56437 \mathrm{E}-01$ $0.56759 E-01$ $0.59953 E-01$ $0.63497 E-01$ $0.65497 E-01$ $0.65323 E-01$ $0.63534 E-01$ $0.61815 \mathrm{E}-01$ $0.60266 E-01$ $0.61108 E-01$ $0.63039 E-01$ $0.64887 E-01$ $0.67204 \mathrm{E}-01$ $0.67655 \mathrm{E}-01$ $0.67259 E-01$ $0.65955 \mathrm{E}-01$ $0.64625 E-01$ $0.64283 E-01$ $0.66361 E-01$ $0.67603 E-01$ $0.68239 \mathrm{E}-01$ $0.67953 E-01$ $0.67790 E-01$ $0.67277 E-01$ $0.67476 \mathrm{E}-01$ C. $67937 \mathrm{E}-01$ $0.67146 E-01$ $0.65554 \mathrm{E}-01$ $0.63850 E-01$
$0.54344 E-02$ $0.53407 \mathrm{E}-02 \quad 0$. $0.52485 \mathrm{E}-02 \quad 0$. $0.51579 E-02 \quad 0$. $0.50689 \mathrm{E}-020$. $0.49814 \mathrm{E}-02 \quad 0$. $0.81306 \mathrm{t}-020$. $0.79902 \mathrm{E}-020$. $0.78523 E-02 \quad 0$. $0.77168 \mathrm{E}-020$. $0.75836 \mathrm{E}-02 \quad 0$. $0.74528 \mathrm{E}-020$. $0.73241 \mathrm{E}-020$. $0.71977 E-02 \quad 0$. $0.70735 \mathrm{E}-02 \quad 0$. $0.69514 \mathrm{E}-020$. $0.68315 E-020$ $0.67136 \mathrm{E}-02 \quad 0$ $0.65977 \mathrm{E}-02 \quad 0$ $0.64838 E-02 \quad 0$. $0.63719 \mathrm{E}-02 \quad 0$. $0.62620 \mathrm{E}-020$ $0.61539 \mathrm{E}-020$ $0.60477 \mathrm{E}-02 \quad 0$ $0.59433 E-02 \quad 0$. $0.58407 \mathrm{E}-02 \quad 0$. $0.57399 \mathrm{E}-020$ 0.56409 E-02 0 . $0.55435 \mathrm{E}-020$. $0.54478 \mathrm{E}-020$. $0.53538 \mathrm{E}-020$ $0.52614 \mathrm{E}-020$. $0.51706 \mathrm{E}-020$ $0.50814 E-020$. $0.49937 E-02 \quad 0$. $0.49192 \mathrm{E}-020$. $0.48343 \mathrm{E}-020$. $0.47508 \mathrm{E}-020$ $0.46688 E-02 \quad 0$ $0.45883 \mathrm{E}-02 \quad 0$. $0.45091 \mathrm{E}-020$. $0.44313 \mathrm{E}-020$ $0.43548 \mathrm{E}-02 \quad 0$. $0.42796 \mathrm{E}-020$. $0.42058 E-02 \quad 0$. $0.41332 \mathrm{E}-020$. $0.40618 \mathrm{E}-02 \quad 0$. $0.39917 E-020$. $0.39228 \mathrm{E}-02 \quad 0$. $0.38552 E-020$. $0.37886 \mathrm{E}-020$. $0.37232 \mathrm{E}-02 \quad 0$. $0.36590 \mathrm{E}-02$ O. $0.35958 \mathrm{E}-020$ $0.35338 E-02 \quad 0$ $\begin{array}{ll}0.34728 \mathrm{E}-02 & 0 . \\ 0.34128 \mathrm{~F}-02 & 0 .\end{array}$ . 
$0.973364 t \quad 00$ $0.102511 E \quad 01$ $0.107511[01$ 0.112691 t 01 $0.106844 \mathrm{E} \quad 01$ $0.925222 E 00$ $0.857347 \mathrm{E} 0 \mathrm{O}$ $0.935955 t$ OC $0.971274 \mathrm{E} \quad 00$ $0.107758 \mathrm{E} \mathrm{OI}$ $0.109584 \mathrm{t} 01$ 0.114393 E 01 $0.110581 E$ OI $0.966878 E$ OO 0.979774 LO $0.993792 \mathrm{E}$ OC 0.101609 E 01 $0.105942 \mathrm{E}$ OI $0.111663 \mathrm{E} 01$ $0.119619 E$ OI $0.134234 E$ OI $0.128264 E$ O1 $0.105501 E 01$ $0.932686 t 00$ $0.925531 \mathrm{E} \mathrm{OO}$ 0.110806 E Ol $0.113153 t 01$ 0.960810 E OD $0.940968 \mathrm{E} C O$ 0.958150 t 00 $0.938164 t \quad 00$ $0.928442 t \quad 00$ $0.934382 \mathrm{E} \quad \mathrm{OO}$ $0.957248 E$ OO $0.983003 E$ OO $0.100427 E$ E 1 $0.107339 E$ D1 $0.107104 E 01$ 0.108360 E O1 $0.111084 t \quad 01$ $0.114108 t_{01}$ $0.115810 \mathrm{E}$ O1 0.114837 E 01 0.110746 E O1 $0.107139 \mathrm{E}$ OI $0.106525 E$ O1 0.106918 E 01 $0.107925 E$ O1 $0.112584 \mathrm{E} O 1$ $0.121551 E$ O1 $0.136167 \mathrm{E}$ O1 $0.153843 E$ OI $0.143045 E \quad 1$ $0.110711 E$ O1 $0.982752 E \quad 00$ 0.102010 E 01 $0.108967 E$ OI
$0.63389 E-01$ $.64573 E-01$ $0.67110 E-01$ $0.70028 E-01$ $0.73729 E-01$ $0.76099 E-01$ $0.74828 \mathrm{E}-01$ $0.70282 E-01$ $0.64265 E-01$ $0.61877 \mathrm{E}-01$ $0.64563 \mathrm{E}-01$ $0.67647 \mathrm{E}-01$ $0.71303 E-01$ $0.74184 E-01$ $0.70542 \mathrm{E}-01$ $0.70946 \mathrm{E}-01$ $0.65842 \mathrm{E}-01$ $0.67723 E-01$ $0.69625 E-01$ $0.71959 \mathrm{E}-01$ $0.74805 E-01$ $0.79033 E-01$ $0.82521 \mathrm{E}-01$ $0.82543 E-01$ $0.78236 \mathrm{E}-01$ C. $69613 \mathrm{E}-01$ $0.68192 E-01$ $0.69463 E-01$ $0.70686 E-01$ $0.69638 E-01$ $0.63225 \mathrm{E}-01$ $0.61162 \mathrm{E}-01$ $0.61379 E-01$ $0.59757 \mathrm{E}-01$ $0.60090 \mathrm{E}-01$ $0.61430 E-01$ $0.63103 E-01$

$0.63631 E-01$

$0.63388 E-01$

$0.63345 E-01$

$0.63883 E-01$ $0.64702 \mathrm{E}-01$ $0.65445 E-01$ $0.65735 E-01$

$0.64989 E-01$

$0.63111 \mathrm{E}-01$ $0.60705 E-01$ $0.58532 \mathrm{E}-01$ $0.57756 \mathrm{E}-01$ $0.57892 E-01$ $0.59120 E-01$ $0.61767 \mathrm{E}-01$ $0.65928 \mathrm{E}-01$ $0.69315 \mathrm{E}-01$

$0.68462 E-01$

$0.62771 E-01$

$0.54442 E-01$ 
$0.134003 \mathrm{E}$ OI $0.153403 \mathrm{E}$ OI $0.126609 E$ OI $0.100521 \mathrm{E} 01$ $0.100881 \mathrm{E} \mathrm{OL}$ $0.103621 E \quad 01$ $0.106514 E$ OI $0.114127 \mathrm{E} 01$ 0.116027 E 01 $0.116339 \mathrm{E} 01$ $0.115788 \mathrm{E} \quad 01$ $0.113911 E \quad 01$ $0.111378 E$ OI $0.109504 \mathrm{E} \quad 01$ $0.109754 \mathrm{E} 01$ $0.111910 \mathrm{E} \mathrm{O1}$ $0.114806 \mathrm{E} \quad 01$ $0.118365 \mathrm{E} \quad 01$ 0.131233 E 01 $0.139816 E$ OI $0.137494 \mathrm{E} \quad 01$ $0.135894 \mathrm{E} \quad 01$ 0.132599 E O1 $0.125688 \mathrm{E}$ OI $0.116584 \mathrm{E}$ OI $0.111708 \mathrm{E} \quad 01$ $0.110747 \mathrm{E} \quad 01$ $0.111117 \mathrm{E}$ OI $0.111758 \mathrm{E}$ OI $0.112401 E$ OI $0.112966 E$ OI $0.113527 E$ O1 $0.114141 \mathrm{E} 01$ $0.114884 \mathrm{E} O 1$ $0.115745 \mathrm{E} 01$ $0.116582 \mathrm{E}$ O1 0.122653 E OI $0.123789 \mathrm{E}$ OI 0.124880 E 01 0.125727 E 01 $0.126092 \mathrm{E} 01$ $0.126292 E^{01}$ $0.126972 \mathrm{E} 01$ 0.128137 E OI $0.129682 \mathrm{E}$ OL $0.131393 E$ OI 0.133667 E 01 0.136470 E O1 $0.139194 \mathrm{E}$ Ol $0.143138 \mathrm{E} \quad 1$ $0.149188 E$ Ol $0.158694 \mathrm{E} \quad 01$ $0.172899 \mathrm{E}$ OI $0.207225 E$ E 1 $0.268763 \mathrm{E} \mathrm{Ol}$ $0.332675 E$ OI

$0.360395 E \quad 01$
$0.281489 E-00$ $0.280828 \mathrm{E}-00$ $0.278598 \mathrm{E}-00$ $0.274128 \mathrm{E}-00$ $0.269832 E-00$ $0.266396 \mathrm{E}-00$ $0.263838 \mathrm{E}-00$ $0.263436 E-00$ $0.262979 E-00$ $0.262365 \mathrm{E}-00$ $0.261575 \mathrm{E}-00$ $0.260575 E-00$ $0.259396 \mathrm{E}-00$ $0.258113 E-00$ $0.256912 \mathrm{E}-00$ $0.255887 \mathrm{E}-00$ $0.255040 E-00$ $0.254391 \mathrm{E}-00$ C. $254383 \mathrm{E}-00$ $0.254625 E-00$ $0.254686 E-00$ $0.254496 E-00$ $0.253954 E-00$ $0.252798 \mathrm{E}-00$ $0.250895 \mathrm{E}-00$ $0.248686 E-00$ $0.246568 E-00$ $0.244712 E-00$ $0.243061 \mathrm{E}-00$ $0.241602 \mathrm{E}-00$ $0.240231 \mathrm{E}-00$ $0.238887 E-00$ $0.23754 C E-00$ $0.236169 \mathrm{E}-00$ $0.234772 \mathrm{E}-00$ $0.233343 \mathrm{E}-00$ $0.231082 \mathrm{E}-0 \mathrm{O}$ $0.228843 E-00$ $0.226637 \mathrm{E}-00$ $0.224467 E-00$ $0.222312 \mathrm{E}-00$ $0.220169 E-00$ $0.218067 \mathrm{E}-00$ $0.216025 \mathrm{E}-00$ 0.21405 CE-0O $0.212136 \mathrm{E}-00$ $0.210295 E-00$ $0.208531 \mathrm{E}-0 \mathrm{C}$ $0.206830 \mathrm{E}-00$ $0.205223 E-00$ $0.203750 \mathrm{E}-00$ $0.202468 \mathrm{E}-00$ $0.201410 \mathrm{E}-00$ $0.200768 \mathrm{E}-00$ $0.200510 \mathrm{E}-00$ $0.200439 \mathrm{E}-00$ $0.200374 \mathrm{E}-00$
$0.50242 E-01$ $0.53270 \mathrm{E}-01$ $0.57452 E-01$ $0.60106 E-01$ $0.58868 E-01$ $0.53153 E-01$ $0.46402 E-01$ $0.46930 E-01$ $0.48300 E-01$ $0.49758 E-01$ $0.50688 E-01$ $0.50841 E-01$ $0.50044 E-01$ $0.48984 E-01$ $0.47935 E-01$ $0.47266 \mathrm{E}-01$ $0.47006 E-01$ $0.46793 E-01$ $0.46726 E-01$ $0.46658 E-01$ $0.47186 E-01$ $0.48909 E-01$

$0.51536 E-01$

$0.54472 E-01$ $0.56100 \mathrm{E}-01$ $0.54944 E-01$ $0.52602 E-01$ $0.49438 E-01$ $0.47145 E-01$ $0.44917 E-01$ $0.43821 E-01$ $0.43452 \mathrm{E}-01$ $0.43482 \mathrm{E}-01$ $0.43854 E-01$ $0.44369 \mathrm{E}-01$

$0.44910 E-01$ $0.45445 E-01$ $0.45720 E-01$ $0.45760 E-01$ $0.45576 \mathrm{E}-01$ $0.45336 E-01$ $0.45074 \mathrm{E}-01$ $0.44720 \mathrm{E}-01$ $0.44389 E-01$ $0.44130 \mathrm{E}-01$ $0.44039 E-01$ $0.44070 E-01$ $0.44214 \mathrm{E}-01$ $0.44463 E-01$ $0.44800 \mathrm{E}-01$

$0.45284 \mathrm{E}-01$ $0.45917 \mathrm{E}-01$

$0.46836 E-01$

$0.48260 \mathrm{E}-01$ $0.50293 \mathrm{E}-01$

$0.53232 \mathrm{E}-01$ $0.56721 E-01$
$0.20467 E-02$ $0.20114 \mathrm{E}-02$ $0.19767 \mathrm{E}-02$ $0.19425 \mathrm{E}-02 \quad 0$. $0.19090 \mathrm{E}-02$ 0. $0.18761 E-02$ O. $0.18437 \mathrm{E}-02$ 0. $0.37480 \mathrm{E}-02$ O. $0.36834 \mathrm{E}-02 \quad 0$. $0.36198 \mathrm{E}-02$ 0. $0.35573 E-02 \quad 0$. $0.34356 \mathrm{E}-02$ 0 . $0.33763 \mathrm{E}-02 \quad 0$. $0.33180 \mathrm{E}-020$. $0.32045 \mathrm{E}-02$ O $0.31492 \mathrm{E}-02$ 02 $0.30948 E-02 \quad 0$. $0.30414 \mathrm{E}-02 \quad 0$. $0.29889 \mathrm{E}-020$. $0.29373 \mathrm{E}-02$ 0. $0.28866 \mathrm{E}-02$ O $0.28368 \mathrm{E}-02$ 0 . $0.27879 \mathrm{E}-02 \quad 0$. $0.27398 \mathrm{E}-02 \quad 0$. $0.26925 \mathrm{E}-02$ 0. $0.26460 \mathrm{E}-02$ 0 . $0.26003 \mathrm{E}-02$ 0 $0.25555 \mathrm{E}-02$ 0 . $0.25114 \mathrm{E}-02 \quad 0$. $0.24680 E-02$ 0 . $0.24254 \mathrm{E}-02$. $0.23836 E-02$ O $0.23424 E-02 \quad 0$ $0.23020 \mathrm{E}-02$ 0 . $0.11127 \mathrm{E}-02 \quad 0$. $0.10935 \mathrm{E}-02 \quad 0$. $0.10747 \mathrm{E}-02$ $0.10561 E-02$ 0. $0.10379 E-02 \quad 0$. $0.10200 E-02$ 0 . $0.10024 \mathrm{E}-020$. $0.98507 \mathrm{E}-03 \quad 0$. $0.96807 \mathrm{E}-03 \quad 0$. $0.95137 \mathrm{E}-03 \quad 0$. $0.93495 \mathrm{E}-030$. $0.91881 \mathrm{E}-030$. $0.90295 E-03 \quad 0$. $0.88737 \mathrm{E}-03 \quad 0$. $0.87206 E-030$. $0.85701 E-030$. $0.84222 \mathrm{E}-03$ O. $0.82768 \mathrm{E}-03 \quad 0$. $0.81339 \mathrm{E}-030$.

$0.79936 E-03 \quad 0$. $0.78556 E-03 \quad 0$ 
7.6596653 7.1823034 26.7131793 26.2521515 25.7990806 24.9162617 24.4862459 24.0636516 23.6483510 23.2402172 22.8391273 22.4449599 22.0575955 21.6769159 21.3028061 20.9351532 20.5738454 20.2187731 19.8698287 19.5269065 19.1899028 18.8587151 18.5332432 18.2133887 17.8990541 17.5901444 7.286566 6.988227 16.4069068 16.1237493 15.8454785 15.5720106 15.3032621 15.0391518 14.7795994 14.5245271 14.2738564 14.0275121 13.7854191 13.5475044 13.3136957 13.0839221 12.8581141 12.6362034 12.4181222 12.2038051 11.9931865 11.7862029 11.5827914 11.3828907 11.1864399 10.9933795 10.8036509

10.6171968

10.4339607
$.688276 \mathrm{E}-01$ $0.987503 E-01$ $0.266113 E-00$ $0.315302 E-00$ $0.305805 E-00$ $0.266681 \mathrm{E}-00$ $0.213636 \mathrm{E}-00$ $0.178931 \mathrm{E}-00$ $0.162722 \mathrm{E}-00$ $0.162104 \mathrm{E}-00$ $0.165977 \mathrm{E}-00$ $0.167966 \mathrm{E}-\mathrm{CO}$ $0.169357 \mathrm{E}-00$ $0.170642 \mathrm{E}-00$ $0.172530 \mathrm{E}-00$ $0.170753 \mathrm{E}-00$ $0.166603 E-00$ $0.161480 \mathrm{E}-00$ $0.156506 \mathrm{E}-00$ $0.151826 \mathrm{E}-00$ $0.147497 \mathrm{E}-00$ $0.144013 E-00$ $0.140662 \mathrm{E}-00$ $0.137243 \mathrm{E}-00$ $0.133689 \mathrm{E}-00$ $0.130146 \mathrm{E}-00$ $0.126955 \mathrm{E}-00$ $0.123213 E-00$ $0.119334 \mathrm{E}-00$ $0.115796 \mathrm{E}-00$ $0.112807 \mathrm{E}-00$ $0.111401 \mathrm{E}-00$ $193 \mathrm{E}-00$ 0.115023E-CO $0.118664 \mathrm{E}-00$ $0.123312 \mathrm{E}-00$ $0.125542 \mathrm{E}-00$ $0.125542 \mathrm{E}-0$ $0.117491 E-00$ $0.111895 \mathrm{E}-\mathrm{CO}$ $0.107586 \mathrm{E}-00$ $0.105143 E-C O$ $0.103726 E-00$ $0.103336 \mathrm{E}$ $0.103403 \mathrm{E}-00$ $0.102925 \mathrm{E}-00$ $0.101888 \mathrm{E}-00$ $0.100844 E-00$ $0.100027 E-00$ $0.988859 \mathrm{E}-01$ $0.973262 \mathrm{E}-\mathrm{Cl}$ $0.953387 E-01$ $0.932944 \mathrm{E}-01$ $0.908618 \mathrm{E}-01$ $0.873069 \mathrm{E}-01$

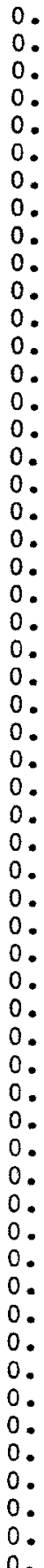

$0.358816 E$ Ol 0.288457 E 01 0.186191 t 01 $0.140843 \mathrm{E} 01$ $0.121825 \mathrm{E} 01$ $0.111605 E$ OI $0.107415 \mathrm{E}$ OI $0.111719 \mathrm{E}$ O1 $0.113656 \mathrm{E} \quad 01$ $0.114871 E$ OI 0.115183 E 01 $0.115009 \mathrm{E} \mathrm{O1}$ $0.115547 E$ OI 0.116519 E Ol $0.117513 \mathrm{E}$ OL $0.118414 E$ O1 $0.119352 E$ O1 $0.120296 \mathrm{E}$ O1 $0.121044 \mathrm{E} 01$ $0.121690 \mathrm{E} 01$ $0.122317 E$ OL $0.123138 E$ O 1 $0.124333 E \quad 01$ 0.125670 E 01 0.127248 E OI $0.129158 E$ O1 $0.131333 E \quad 01$ $0.133736 \mathrm{E} \quad 01$ $0.136185 \mathrm{E} \mathrm{OL}$ 0.139078 E 01 $0.142370 \mathrm{E} 01$ $0.145621 E 01$ $0.148703 \mathrm{E} \quad 01$ $0.150783 t \quad 01$ 0.150889 t 01 $0.148947 \mathrm{E} \quad 01$ $0.148778 E$ O 1 0.144271 E 01 $0.140493 E_{01}$ $0.139286 \mathrm{E} \quad 01$ $0.139756 E$ OI $0.140890 \mathrm{E}$ O1 $0.142242 E \quad 01$ $0.143063 E \quad 01$ 0.143381 E 01 $0.143138 \mathrm{E}$ 01 $0.142420 E \quad 01$ 0.141926 E 01 $0.141740 E$ OI $0.141430 \mathrm{E} \quad 01$ $0.140871 \mathrm{E}$ O1 $0.140440 \mathrm{E} 01$ $0.140213 E$ O $0.140231 \mathrm{E} 01$ $0.140469 \mathrm{E} 01$ $0.141215 \mathrm{E} 01$ $0.143272 \mathrm{E}$ OI $.199648 E-00$ $0.197872 \mathrm{E}-00$ $0.194776 E-00$ $0.190878 \mathrm{E}-00$ $0.187051 \mathrm{E}-00$ $0.183771 E-00$ $0.180953 \mathrm{E}-00$ $0.178767 \mathrm{E}-00$ $0.176866 \mathrm{E}-00$ $0.174958 \mathrm{E}-00$ $0.172959 \mathrm{E}-00$ 0.170907 E-00 $0.168812 \mathrm{E}-0$ $0.166679 \mathrm{E}-00$ $0.164496 E-00$ $0.162291 E-00$ $0.160111 \mathrm{E}-00$ $0.157994 \mathrm{E}-00$ $0.155956 \mathrm{E}-00$ $0.153996 E-00$ $0.152107 \mathrm{E}-00$ $0.150286 E-00$ $0.148516 \mathrm{E}-00$ $0.146795 \mathrm{E}-00$ $0.145127 \mathrm{E}-00$ $0.143513 E-00$ $0.141953 \mathrm{E}-00$ $0.140442 E-00$ $0.138989 E-00$ $0.137599 \mathrm{E}-00$ $0.136263 E-00$ $0.134972 \mathrm{E}-00$ $0.133696 \mathrm{E}-00$ $0.132393 \mathrm{E}-00$ $0.131024 \mathrm{E}-00$ $0.129464 \mathrm{E}-00$ $0.127801 \mathrm{E}-00$ $0.126082 E-00$ $0.124401 E-00$ $0.122809 \mathrm{E}-00$ $0.121311 E-00$ $0.119884 \mathrm{E}-00$ $0.118494 \mathrm{E}-00$ $0.117121 \mathrm{E}-00$ $0.115746 \mathrm{E}-00$ $0.114359 \mathrm{E}-00$ $0.112972 \mathrm{E}-00$ $0.111595 \mathrm{E}-00$ $0.110228 \mathrm{E}-00$ $0.108868 \mathrm{E}-00$ $0.107519 \mathrm{E}-00$ $0.106191 t-00$ $0.104891 \mathrm{E}-00$ $0.103622 \mathrm{E}-00$ $0.102390 \mathrm{E}-00$ $0.101218 \mathrm{E}-00$

$61039 E-01$ $0.66749 E-01$ $.65638 \mathrm{E}-01$ $0.50471 E-01$ $0.41085 \mathrm{E}-01$ $0.33884 E-01$ $0.29186 \mathrm{E}-01$ $0.27031 \mathrm{E}-01$ $0.27033 \mathrm{E}-01$ $0.27665 \mathrm{E}-01$ $0.28319 E-01$ 
0.143999 E 01 $0.142968 E$ O1 $0.142907 E$ OI 0.143677 E 01 0.144765 E 01 $0.145948 E$ O1 $0.147167 t 01$ $0.148395 \mathrm{E} \mathrm{OI}$ $0.152294 \mathrm{E}$ OI $0.156364 t 01$ $0.159162 E \quad 01$ 0.162301 E 01 $0.166583 \mathrm{E} \mathrm{Ol}$ 0.171760 E 01 $0.176193 \mathrm{E} \mathrm{O1}$ 0.181958 E 01 0.188892 E 01 $0.196209 \mathrm{E} 01$ 0.203749 E 01 0.209093 E CI $0.210814 t 01$ $0.211345 t 01$ 0.211976 E 01 $0.211835 \mathrm{E} 01$ 0.206402 E 01 $0.200473 E 01$ $0.186371 E$ OI $0.173410 \mathrm{E} \mathrm{Cl}$ 0.174259 E 01 $0.174948 \mathrm{E} 01$ 0.176673 E 01 0.179020 E OI $0.180134 t$ Ol $0.179855 E$ OI 0.179828 E 01 $0.179642 E 01$ 0.177751 E OI 0.175165 E 01 $0.172941 \mathrm{E} \mathrm{Ol}$ $0.172205 t$ ol 0.170838 t 01 $0.169186 \mathrm{E} 01$ $0.168324 t$ OI $0.168119 t$ OI 0.167809 E O1 $0.167631 \mathrm{E} \mathrm{Ol}$ 0.169279 E 01 $0.170961 \mathrm{E} \mathrm{OI}$ $0.170501 E$ OI $0.169636 \mathrm{E} \mathrm{Ol}$ $0.170288 E$ OI $0.172571 E 01$ $0.174731 \mathrm{E} \mathrm{OI}$ $0.175320 t$ ol 0.175918 E 01 $\begin{array}{lll}0.175918 E & 01 \\ 0.183898 E & 01\end{array}$ $0.186061 \mathrm{E} \mathrm{O1}$
$0.100062 E-00$ $0.988898 E-01$ $0.977223 E-01$ $0.965774 \mathrm{E}-01$ $0.954701 \mathrm{E}-01$ $0.943935 \mathrm{E}-01$ $0.933426 \mathrm{E}-01$ $0.923158 t-01$ $0.913099 \mathrm{E}-01$ G. $90354 \mathrm{CE}-01$ $0.894294 \mathrm{E}-01$ $0.885315 \mathrm{E}-01$ $0.876690 \mathrm{E}-01$ $0.868497 \mathrm{E}-01$ $0.860582 E-01$ $0.853056 \mathrm{E}-01$ $0.84592 \mathrm{CE}-01$ $0.839182 \mathrm{E}-01$ $0.832784 E-01$ $0.826534 E-01$ $0.820237 \mathrm{E}-01$ $0.813865 \mathrm{E}-01$ $0.807459 \mathrm{E}-01$ C. $801018 \mathrm{E}-01$ $0.794227 \mathrm{E}-01$ $0.787136 \mathrm{E}-01$ $0.778988 \mathrm{E}-01$ $0.770070 E-01$ $0.761669 \mathrm{E}-\mathrm{O} 1$ $0.753676 E-01$ $0.746331 \mathrm{E}-01$ $0.739247 \mathrm{E}-01$ $0.732346 E-01$ $0.725485 E-01$ $0.718649 E-01$ $0.71181 \mathrm{CE}-01$ $0.704857 E-01$ $0.697753 E-01$ $0.690536 E-01$ $0.683377 E-01$ $0.676220 \mathrm{E}-01$ $0.66909 C E-01$ $0.662048 \mathrm{E}-01$ $0.655158 t-01$ $0.648404 E-01$ $0.641778 \mathrm{E}-01$ $0.635392 \mathrm{E}-01$ $0.629195 \mathrm{E}-01$ $0.623019 \mathrm{E}-01$ $0.616833 E-01$ $0.610741 \mathrm{E}-01$ $0.604881 \mathrm{E}-01$ $0.599221 E-01$ $0.593629 \mathrm{E}-01$ $0.588116 \mathrm{E}-01$ $0.579614 \mathrm{E}-01$
$0.571431 \mathrm{E}-01$ $0.20920 E-01$ $0.20780 E-01$ $0.20602 E-01$ $0.20286 \mathrm{E}-01$ $0.20075 \mathrm{E}-01$ $0.19927 E-01$ $0.19794 \mathrm{E}-01$ $0.19670 \mathrm{E}-01$ $0.19609 \mathrm{E}-01$ $0.19643 \mathrm{E}-01$ $0.19731 E-01$ $0.19937 E-01$ $0.20012 E-01$ $0.20221 E-01$ $0.20398 E-01$ $0.20657 E-01$ $0.20860 E-01$ $0.21122 E-01$ $0.21423 E-01$ $0.21695 E-01$ $0.21895 \mathrm{E}-01$ $0.22001 \mathrm{E}-01$ $0.21975 E-01$ $0.21878 \mathrm{E}-01$ $0.21706 \mathrm{E}-01$ $0.21387 E-01$ $0.20868 \mathrm{E}-01$ $0.19954 E-01$ $0.19198 E-01$ $0.18161 \mathrm{E}-01$ $0.17924 E-01$ $0.17659 \mathrm{E}-01$ $0.17457 \mathrm{E}-01$ $0.17379 \mathrm{E}-01$ $0.17274 \mathrm{E}-01$ $0.17172 \mathrm{E}-01$ $0.17022 \mathrm{E}-01$ C.16874E-01 $0.16614 \mathrm{E}-01$ C. $16334 \mathrm{E}-01$ $0.16008 \mathrm{E}-01$ $0.15679 E-01$ $0.15344 \mathrm{E}-01$ $0.14990 \mathrm{E}-01$ $0.14710 \mathrm{E}-01$ $0.14471 E-01$ $0.14288 \mathrm{E}-01$ $0.14132 \mathrm{E}-01$ $0.14022 \mathrm{E}-01$ $0.13906 \mathrm{E}-01$ $0.13743 E-01$ C. $13565 \mathrm{E}-01$ $0.13462 \mathrm{E}-01$

$0.13358 \mathrm{E}-01$

$0.13254 \mathrm{E}-01$

$0.13036 \mathrm{E}-01$
$0.42196 E-03$ $0.41468 E-03$ $0.40752 E-03$ $0.4 C 049 E-03$ $0.39357 \mathrm{E}-03$ $0.38678 \mathrm{E}-03$ $0.37355 \mathrm{E}-03$ $0.23599 E-03$ $0.23191 \mathrm{E}-03 \mathrm{O}$ $0.22791 \mathrm{E}-03$ $0.22011 E-03$ $0.21631 \mathrm{E}-03$ $0.21258 \mathrm{E}-03$ $0.20891 E-03$ $0.20531 \mathrm{E}-03$ $0.20176 \mathrm{E}-03$ $0.19828 \mathrm{E}-03$ $0.19486 E-03$ $0.19150 \mathrm{E}-03$ $0.18819 \mathrm{E}-03$ $0.18494 \mathrm{E}-03$ $0.18175 \mathrm{E}-03$ $0.17861 E-03$ $0.17553 E-03$ $0.17250 \mathrm{E}-03$ $0.16953 E-03$ $0.16660 \mathrm{E}-03$ $0.16372 \mathrm{E}-03$ $0.16090 \mathrm{E}-03$ $0.15812 \mathrm{E}-03$ $0.15539 \mathrm{E}-03$ $0.15271 \mathrm{E}-03$ $0.15008 \mathrm{E}-03$ $0.14748 E-03$ $0.14494 E-03$ $0.14244 \mathrm{E}-03$ $0.13998 \mathrm{E}-03$ $0.13756 \mathrm{E}-03$ $0.13519 E-03$ $0.13286 E-03$ $0.13056 E-03$ $0.12831 \mathrm{E}-03$ $0.12610 \mathrm{E}-03$ $0.12392 \mathrm{E}-03$ $0.12178 \mathrm{E}-03$ $0.11968 E-03$ $0.11761 \mathrm{E}-03$ $0.11558 \mathrm{E}-03$ $0.11359 E-03$

$0.11163 \mathrm{E}-03$ $0.10970 E-03$ $0.10781 \mathrm{E}-03$ $0.10595 \mathrm{E}-03$ $0.14932 \mathrm{E}-03$ $0.14675 E-03$ 
$\begin{array}{llll}0.187574 t & 01 & 0.563515 E-01 & 0.12812 E-01\end{array}$ $0.188227 \mathrm{E} 01$ $0.191816 \mathrm{E} \mathrm{Cl}$ 0.201060 E OI $0.211693 t 01$ $0.225538 E$ OI $0.241824 \mathrm{E} \quad 01$ $0.252199 \mathrm{E}$ OI $0.261658 E$ OI $0.279692 \mathrm{E}$ OI $0.295334 \mathrm{E} \quad 01$ $0.314822 \mathrm{E} \quad 01$ $0.340995 t \quad 01$ $0.396873 t$ OI $0.489745 \mathrm{E}$ OI $0.586901 \mathrm{E}$ O1 $0.560142 \mathrm{E} 01$ $0.424895 \mathrm{E}$ OI $0.318330 E$ OI $0.272991 E$ OI 0.260695 E 01 $0.222108 E$ Ol $0.184501 E$ OI $0.170010 \mathrm{E} \mathrm{OL}$ 0.164729 E 01 $0.161012 E$ OI $0.158314 \mathrm{E} 01$ 0.157166 E 01 $0.156528 E \quad 01$ $0.155714 \mathrm{E} \quad 1$ 0.155101 t 01 $0.154694 \mathrm{E}$ 01 $0.154236 \mathrm{E} 01$ $0.153804 \mathrm{E} 01$ 0.153553 t 01 $0.153504 \mathrm{E} \quad 1$ $0.153515 E$ OI $0.153455 \mathrm{E} \mathrm{O1}$ $0.153466 \mathrm{E} \mathrm{OI}$ $0.153477 \mathrm{E}$ OI $0.153366 E$ O 1 $0.153261 \mathrm{E} 01$ 0.153220 E 01 $0.153196 \mathrm{E} \mathrm{Cl}$ $0.153295 \mathrm{E} \quad 01$ $0.153417 E \quad 01$ $0.153426 E$ CI $0.153516 t 01$ $0.153605 t 01$ $0.153568 \mathrm{E} 01$ $0.153593 E \quad 01$ 0.153649 E 01 0.153659 E 01 0.153715 E C1 0.153789 E Cl $0.153881 \mathrm{E}$ O1 $0.154019 E \quad 1$
$55579 \mathrm{CE}-01$

$0.548496 E-01$ $.541641 E-01$ . $535521 \mathrm{E}-01$ $0.530177 \mathrm{E}-01$ C. $525571 \mathrm{E}-01$ $0.521431 \mathrm{E}-01$ $0.51761 \varepsilon \mathrm{E}-01$ $0.514227 \mathrm{E}-01$ $0.511149 E-01$ $0.508324 \mathrm{E}-01$ $0.505804 E-01$ $0.50370 \mathrm{CE}-01$ $0.502126 E-01$ $0.500909 \mathrm{E}-01$ $0.499793 \mathrm{E}-01$ $0.498113 E-01$ $0.495498 \mathrm{E}-01$ $0.492008 E-01$ $0.488213 \mathrm{E}-01$ $0.483287 t-01$ $0.476609 \mathrm{E}-01$ $0.468653 E-01$ $0.460089 \mathrm{E}-01$ $0.451098 \mathrm{E}-01$ $0.442020 E-01$ $0.433082 \mathrm{E}-01$ $0.424382 E-01$ $0.41582 C E-01$ $0.407554 \mathrm{E}-01$ C. $399639 \mathrm{E}-01$ $0.391913 \mathrm{E}-01$ $0.384296 \mathrm{E}-01$ $0.376775 E-01$ $0.369344 \mathrm{E}-\mathrm{Cl}$ $0.361999 \mathrm{E}-01$ $0.354754 \mathrm{E}-01$ $0.347636 t-01$ $0.340654 \mathrm{E}-01$ $0.333805 \mathrm{E}-01$ $0.327091 \mathrm{E}-01$ $0.320516 E-01$ $0.314077 t-01$ $0.30778 C \mathrm{E}-01$ $0.301615 \mathrm{E}-01$ C. $295567 \mathrm{E}-01$ $0.289638 \mathrm{E}-01$ $0.283823 \mathrm{E}-01$ $0.278112 \mathrm{E}-01$ $0.272512 \mathrm{E}-01$ $0.26702 \mathrm{CE}-01$ $0.261636 E-01$ $0.256362 \mathrm{E}-01$ $0.251198 \mathrm{E}-01$ $0.246143 \mathrm{E}-01$ $0.241198 \mathrm{E}-01$ $0.14173 \mathrm{E}-03$ O. $0.13928 E-03$ O. $0.13688 E-03 \quad 0$. $0.13451 \mathrm{E}-03 \quad 0$. $0.13219 \mathrm{E}-03 \quad 0$. $0.12991 \mathrm{E}-03 \quad 0$. $0.12767 \mathrm{E}-03 \quad 0$ $0.12546 \mathrm{E}-03 \mathrm{O}$ $0.12330 \mathrm{E}-030$. $0.12117 E-03 \quad 0$. $0.11908 \mathrm{E}-03 \quad 0$. $0.11703 E-030$. $0.11501 E-03$. $0.11302 E-03 \quad 0$ $0.11107 E-03 \quad 0$ $0.10915 \mathrm{E}-030$. $0.10727 E-03 \quad 0$. $0.10542 \mathrm{E}-03 \quad 0$. $0.10360 E-030$. $0.1 C 181 \mathrm{E}-030$ $0.10005 E-030$. $0.98327 E-04 \quad 0$. $0.96630 E-04 \quad 0$. $0.94962 E-04 \quad 0$. $0.93323 E-04 \quad 0$ $0.91713 \mathrm{E}-04 \quad 0$. $0.90130 \mathrm{E}-040$. $0.88574 \mathrm{E}-04 \quad 0$. $0.87046 E-04 \quad 0$. $0.85543 \mathrm{E}-04 \mathrm{0}$ $0.84067 \mathrm{E}-04 \quad 0$. $0.82616 \mathrm{E}-04 \quad 0$. $0.81190 \mathrm{E}-04 \quad 0$. $0.79789 E-04 \quad 0$. $0.78412 E-04 \quad 0$. $0.77059 E-04 \quad 0$. $0.75729 E-04 \quad 0$. $0.74422 E-040$. $0.73138 E-04 \quad 0$. $0.71875 \mathrm{E}-04 \quad 0$ $0.70635 E-04 \quad 0$. $0.69416 E-040$. $0.6 F 218 \mathrm{E}-04 \quad 0$. $0.67040 \mathrm{E}-040$. $0.65883 E-04 \quad 0$. $0.64746 E-040$. $0.63629 E-04 \quad 0$. $0.62531 \mathrm{E}-04 \quad 0$. $0.61452 \mathrm{E}-040$. $0.60391 \mathrm{E}-04 \quad 0$. $0.59349 E-04 \quad 0$. $0.58325 E-04 \quad 0$. $0.57318 E-04 \quad 0$. $0.56329 E-04 \quad 0$. $0.55357 \mathrm{E}-04 \quad 0$. $0.54401 E-04$ 


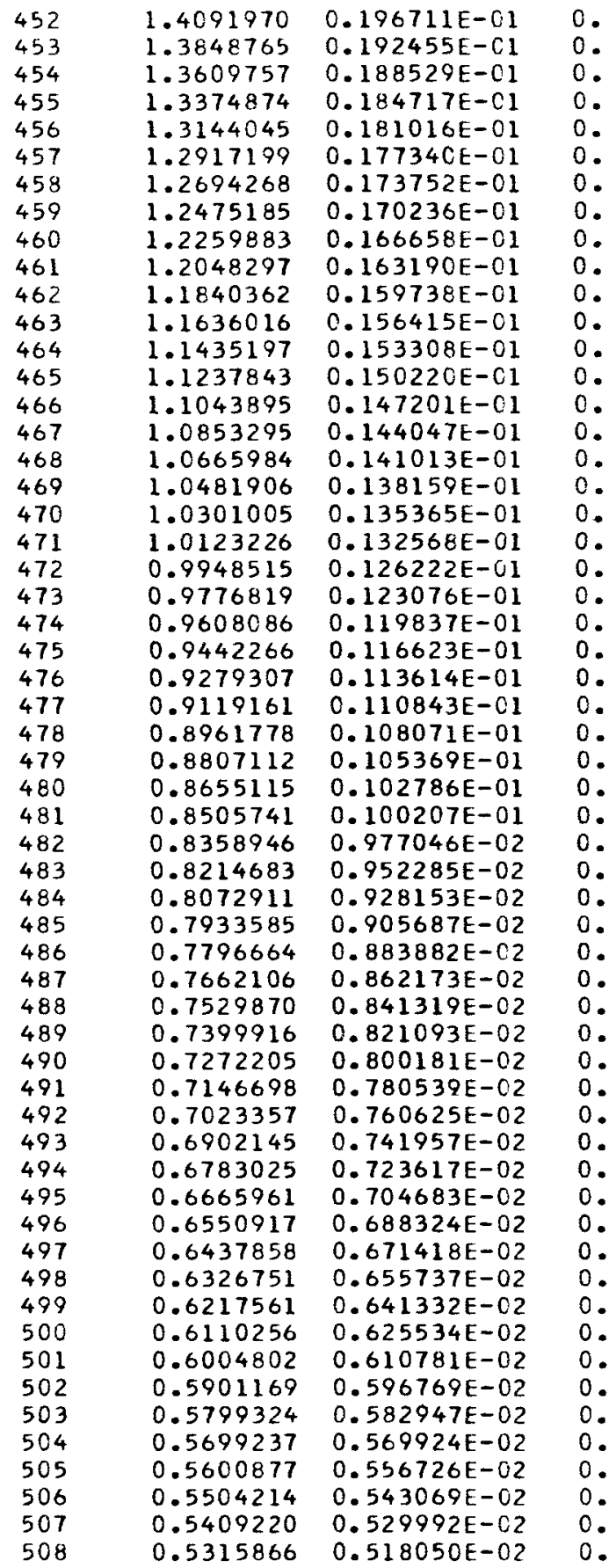

$0.154238 \mathrm{E} \quad 01$ $0.154457 \mathrm{E}$ OI $0.154595 E$ OI $0.154733 E$ OI $0.154870 E$ OI $0.155008 E$ OI $0.155146 E$ OI $0.155284 \mathrm{E}$ OI $0.155483 E$ OI $0.155682 E \quad 01$ $0.155901 \mathrm{E}$ OI $0.156120 \mathrm{E} 01$ $0.156257 \mathrm{E} \mathrm{OI}$ $0.156401 \mathrm{E} \mathrm{CI}$ 0.156557 E C1 $0.156801[01$
$0.157044 E$ $\begin{array}{lll}0.157044 E & 01 \\ 0.157206 E & 01\end{array}$ $0.157368 t \quad 01$ $0.157576 t$ ol $0.176017 \mathrm{E}$ O C.176179E CI $0.176407 \mathrm{E}$ OI $0.176615 E \quad 01$ 0.176778 E OI 0.176940 E 21 $0.177148 E$ OI $0.177356 t \quad 01$ $0.177518 E$ OI 0.177726 E 01 $0.177934 E$ OI $0.178142 \mathrm{E} \quad 01$ $0.178350 E$ E 0.178431 E OI $0.178477 \mathrm{E} \mathrm{OI}$ $0.178603 E \quad 01$ $0.178684 \mathrm{t}$ OI $0.178725 \mathrm{E}$ OI 0.178927 E 0 $0.179089 \mathrm{E} \mathrm{OI}$ $0.179297 \mathrm{E}$ OI 0.179505 E 01 $0.179713 E$ O $0.180083 E$ O $0.180245 E \quad 01$ $0.180453 \mathrm{E}$ O 0.180661 E 01 $0.180661 \mathrm{E} \mathrm{O}$ 0.180869 E 01 $0.181077 \mathrm{E}$ OI $0.181123 E$ OI $0.181215 E$ OI $0.181261 \mathrm{E} 01$ $0.181306 \mathrm{E} \quad 01$ $0.181514 \mathrm{E} C$ $0.181722 \mathrm{E} \quad \mathrm{C}$ $0.181768 \mathrm{E} \mathrm{O} 1$
$0.236364[-01$ $0.231639 E-01$ $0.227011 \mathrm{E}-01$ $0.222479 E-01$ $0.218039 \mathrm{E}-01$ $0.213691 \mathrm{E}-01$ $0.209433 \mathrm{E}-01$ $0.205263 E-01$ $0.201182 E-01$ $0.19718 E E-01$ C. $193281 \mathrm{E}-01$ $0.189458 E-01$ $0.185711 \mathrm{E}-01$ $0.182042 \mathrm{E}-01$ $0.178447 \mathrm{E}-01$ $0.174932 \mathrm{E}-0$ $0.171492 \mathrm{E}-01$ $0.168124 E-01$ $0.164825 E-01$ $0.161596 \mathrm{E}-01$ $0.157517 \mathrm{E}-01$ $0.153548 E-01$ $0.149693 E-01$ $0.145953 \mathrm{E}-01$ $0.142319 \mathrm{E}-0$ C. $138781 \mathrm{E}-01$ $0.135339 \mathrm{E}-01$ 0.131992 E-0 1 $0.128734 \mathrm{E}-01$ $0.125566 E-01$ $0.122485 \mathrm{E}-01$ $0.119489 \mathrm{E}-01$ $0.116577 \mathrm{E}-01$ $0.113742 \mathrm{E}-01$ $0.110981 \mathrm{E}-0$ $0.108295 E-01$ $0.1 C 568 C E-0$ $0.103134 \mathrm{E}-01$ $0.100659 \mathrm{E}-01$ $0.982515 \mathrm{E}-02$ $0.959121 \mathrm{E}-02$ $0.93636 \mathrm{CE}-02$ $0.914222 \mathrm{E}-02$ $0.892733 \mathrm{E}-02$ $0.871790 \mathrm{E}-02$ $0.851416 E-02$ $0.831564 E-02$ $0.812182 E-C 2$ $0.793331 \mathrm{E}-02$ C. $774969 \mathrm{E}-02$ $0.757067 \mathrm{E}-02$ $0.73962 \mathrm{CE}-02$ $0.722596 E-02$ $0.706006 \mathrm{E}-02$ C. $689869 \mathrm{E}-\mathrm{C} 2$ $0.674164 E-02$ $0.658846 \mathrm{E}-02$
$0.52681 E-02$ $0.51655 E-02$ $0.50674 \mathrm{E}-02$ C. $49721 \mathrm{E}-02$ $0.48795 \mathrm{E}-02$ $0.47873 E-02$ $0.46972 \mathrm{E}-02$ $0.46088 \mathrm{E}-02$ $0.45217 \mathrm{E}-02$ $0.44364 \mathrm{E}-02$ $0.43531 \mathrm{E}-02$ $0.42718 \mathrm{E}-02$ $0.41929 E-02$ $0.41146 E-02$ $0.40385 \mathrm{E}-02$ $0.39625 \mathrm{E}-02$ $0.38885 \mathrm{E}-02$ $0.38162 \mathrm{E}-02$ $0.37454 E-02$ $0.36762 \mathrm{E}-02$ $0.36073 E-02$ $0.35226 E-02$ $0.34370 \mathrm{E}-02$ $0.33507 \mathrm{E}-02$ $0.32687 \mathrm{E}-02$ $0.31935 E-02$ C. $31196 \mathrm{E}-02$ $0.30471 E-02$ $0.29765 \mathrm{E}-02$ $0.29074 E-02$ C. $28398 \mathrm{E}-02$ $0.27731 \mathrm{E}-02$ C. $27075 \mathrm{E}-02$ $0.26432 \mathrm{E}-02$ $0.25803 E-02$ $0.25196 E-02$ $0.24598 \mathrm{E}-02$ $0.24012 \mathrm{E}-02$ $0.23445 E-02$ $0.22895 E-02$ $0.22357 \mathrm{E}-02$ C. $21841 \mathrm{E}-02$ $0.21346 \mathrm{E}-02$ $0.20856 E-02$ $0.20395 E-02$ $0.19936 \mathrm{E}-02$ $0.19500 \mathrm{E}-02$ $0.19068 E-02$ $0.18637 \mathrm{E}-02$ $0.18225 \mathrm{E}-02$ $0.17812 \mathrm{E}-02$ $0.17411 E-02$ $0.17025 E-02$

$0.16636 E-02$

C. $16259 \mathrm{E}-02$

$0.15893 E-02$

$0.15538 \mathrm{E}-02$
$0.53462 E-04$ $0.52540 E-04$ $0.51633 \mathrm{E}-04 \quad 0$. $0.50742 \mathrm{E}-04 \quad 0$ $0.49866 \mathrm{E}-04 \quad 0$ $0.49006 E-04 \quad 0$ $0.48160 \mathrm{E}-04 \quad 0$. $0.47329 E-04 \quad 0$. $0.46512 \mathrm{E}-04 \quad 0$ $0.45709 E-04 \quad 0$ $0.44920 E-04 \quad 0$. $0.44145 E-04 \quad 0$ $0.43383 E-04 \quad 0$. $0.42634 \mathrm{E}-04 \quad 0$. $0.41899 E-04 \quad 0$ $0.41175 E-04 \quad 0$. $0.40465 E-04 \quad 0$. $0.39766 E-04 \quad 0$. $0.39080 E-04 \quad 0$. $0.38406 E-04 \quad 0$ $0.11325 E-03 \quad 0$. $0.11130 E-03 \quad 0$ $0.10938 \mathrm{E}-03 \quad 0$. $0.10749 E-03 \quad 0$. $0.10563 E-03$ O. $0.10381 E-03 \quad 0$. $0.10202 E-03 \quad 0$. $0.10026 E-030$. $0.98529 \mathrm{E}-040$. $0.96828 E-04 \quad 0$ $0.95157 E-04 \quad 0$. $0.93515 E-04 \quad 0$ $0.91901 E-04 \quad 0$. $0.90315 E-04 \quad 0$. $0.88756 \mathrm{E}-04 \quad 0$. $0.87224 E-04 \quad 0$ $0.85719 E-04 \quad 0$ $0.84240 E-04 \quad 0$ $0.82786 E-04 \quad 0$. $0.81357 \mathrm{E}-04 \quad 0$. $0.79953 E-04 \quad 0$. $0.78573 \mathrm{E}-04 \quad 0$. $0.77217 \mathrm{E}-04 \quad 0$ $0.75884 E-04 \quad 0$. $0.74575 E-04 \quad 0$. $0.73288 E-04 \quad 0$. $0.72023 E-040$. $0.70780 E-04 \quad 0$. $0.69558 \mathrm{E}-04 \quad 0$. $0.68358 E-040$. $0.67178 E-040$. $0.66029 E-04 \quad 0$. $0.64879 \mathrm{E}-04 \quad 0$. $0.63760 E-04 \quad 0$. $0.62659 E-04 \quad 0$ $0.61578 E-04 \quad 0$.

$0.60515 E-040$ 

$0.329326 \mathrm{E}-\mathrm{C} 2$ $0.3688076 \quad 0.322351 \mathrm{E}-02$ $0.3624425 \quad 0.315405 E-02$ $0.3561874 \quad 0.308740 E-02$ 0.3500401 0.3439990 0.3380621 $0.302186 E-02$ $0.295808 \mathrm{E}-02$ $0.289583 E-02$

$0.283298 \mathrm{E}-\mathrm{C} 2$

$0.277199 \mathrm{E}-02$

$0.3208592 \quad 0.270885 \mathrm{E}-02$

$0.3153216 \quad 0.264880 E-02$

$0.3098797 \quad 0.259422 \mathrm{E}-02$

$0.3045316 \quad 0.254061 \mathrm{E}-02$

$0.2992759 \quad 0.219682 \mathrm{E}-02$

$0.2941109 \quad 0.210041 \mathrm{E}-02$

$0.2890350 \quad 0.200518 \mathrm{E}-02$

$0.284 C 467 \quad 0.190784 \mathrm{E}-02$

$0.2791445 \quad 0.181918 \mathrm{E}-02$

$0.27432690 .174519 \mathrm{E}-02$

$0.2695924 \quad 0.167496 E-02$

$0.2649397 \quad 0.160788 E-02$

$0.2603673 \quad 0.154397 \mathrm{E}-02$

$0.2558737 \quad 0.148097 \mathrm{E}-02$

$0.2514577 \quad 0.141747 \mathrm{E}-02$

$0.2471180 \quad 0.135643 \mathrm{E}-02$

$0.2428531 \quad 0.129824 \mathrm{E}-02$

$0.2386618 \quad 0.123992 \mathrm{E}-\mathrm{C2} 2$

$0.2345429 \quad 0.118499 \mathrm{E}-02$

$0.2304951 \quad 0.113498 \mathrm{E}-02$

$0.2265171 \quad 0.108814 E-02$

$0.2226078 \quad 0.104389 \mathrm{E}-02$

$0.2187659 \quad 0.100187 \mathrm{E}-02$

$0.2149903 \quad 0.961690 E-03$

$0.2112799 \quad 0.923373 E-03$

$0.2076336 \quad 0.886756 E-03$

$0.2040502 \quad 0.851716 E-C 3$

$0.2005286 \quad 0.818182 \mathrm{E}-\mathrm{C} 3$

0.1970678

$0.786132 \mathrm{E}-\mathrm{C} 3$

$0.385901 \mathrm{E}-02$

$0.377619 E-0$ $0.183575 \mathrm{E} 01 \quad 0.369526 \mathrm{E}-02$ $0.183667 \mathrm{E}$ O1 $0.353912 \mathrm{E}-02$ $0.183829 \mathrm{E}$ O $0.183991 \mathrm{E}$ OI $0.183991 E$ OI $0.183991 E$ OI $0.207491 E$ OI $0.207861 \mathrm{E}$ OI $0.208023 E$ OI $0.208023 E$ OI $0.208185 E \quad 01$ $0.208347 E \quad 01$ $0.208347 E$ OI $0.208347 \mathrm{E} \quad 01$ $0.208347 E$ OI $0.208347 \mathrm{E} \mathrm{OI}$ 0.208509 E CI 0.208671 t 01 $0.208671 \mathrm{E} \mathrm{OI}$ $0.208753 E$ OI $0.208915 \mathrm{E} \mathrm{OI}$ $0.208996 E$ OI 0.208996 t 01 C. $208996 \mathrm{E} \mathrm{OI}$ C. $208996 \mathrm{E} 01$ C. 208996 E 31 C.208996E 01 C. $208996 t 01$ $0.208996 E$ OI $0.346394 E-02$ $0.339062 \mathrm{E}-02$ $0.331892 \mathrm{E}-02$ $0.324883 \mathrm{E}-02$ $0.310097 \mathrm{E}-02$ $0.296029 t-02$ $0.282669 \mathrm{E}-02$ $0.270035 \mathrm{E}-02$ $0.258060 \mathrm{E}-02$ $0.246628 \mathrm{E}-02$ $0.235710 E-02$ $0.225282 \mathrm{E}-02$ $0.215319 \mathrm{E}-02$ $0.205815 E-02$ $0.196775 \mathrm{E}-02$ $0.188180 E-02$ $0.180008 \mathrm{E}-02$ $0.172261 E-02$ $0.164912 \mathrm{E}-02$ C. $157924 E-02$ $0.151271 \mathrm{E}-02$ $0.144935 E-02$ $0.138897 \mathrm{E}-02$ C. $133144 \mathrm{E}-02$ $0.127662 \mathrm{E}-02$ $0.12243 E \mathrm{E}-\mathrm{J} 2$ $0.117460 \mathrm{E}-02$ $0.208996 \mathrm{E} 01$ 0.108196E-02 $0.13306 \mathrm{E}-02$ $0.13015 E-02$ $0.12735 E-02$ $0.12456 \mathrm{E}-02$ $0.12186 \mathrm{E}-02$ $0.11923 E-02$ $0.11667 E-02$ $0.11411 \mathrm{E}-02$ $0.11162 \mathrm{E}-02$ $0.10919 E-02$ $0.10679 E-02$ $0.10447 \mathrm{E}-02$ $0.10220 \mathrm{E}-02$ $0.10000 E-02$ $0.97869 E-03$ $0.95792 E-03$ $0.93787 E-03$ $0.91780 E-03$ $0.89827 E-03$ $0.87922 \mathrm{E}-03$ $0.86040 E-03$ $0.84204 E-03$ $0.82413 E-03$ $0.80676 \mathrm{E}-03$ $0.79001 E-03$ $0.77357 \mathrm{E}-03$ $0.75768 E-03$ $0.72599 E-03$ $0.69318 E-03$ $0.65875 E-03$ $0.62842 E-03$ $0.60301 E-03$ $0.57821 E-03$ $0.55452 \mathrm{E}-03$ $0.53197 \mathrm{E}-03$ $0.50974 E-03$ $0.48810 E-03$ $0.46707 E-03$ $0.44649 E-03$ $0.42620 E-03$ $0.40733 E-03$ $0.38986 E-03$ $0.37330 E-03$ $0.35767 \mathrm{E}-03$ $0.34283 E-03$ $0.32866 \mathrm{E}-03$ $0.31514 E-03$ $0.30224 E-03$ $0.28990 \mathrm{~F}-03$ $0.27810 \mathrm{E}-03$ $0.26683 E-03$

$.59471 \mathrm{E}-04 \quad 0$. $0.56444 \mathrm{E}-04 \quad 0$ $0.55470 E-04 \quad 0$. $0.54513 E-04 \quad 0$. $0.53572 \mathrm{E}-04 \quad 0$ $0.52648 E-04 \quad 0$. $0.51739 E-04 \quad 0$ $0.50846 E-040$. $0.49968 \mathrm{E}-04 \quad 0$. $0.49106 E-04 \quad 0$. $0.48259 E-04 \quad 0$. $0.47426 \mathrm{E}-04 \quad 0$. $0.46607 \mathrm{E}-040$. $0.45803 E-04 \quad 0$. $0.45012 \mathrm{E}-040$. $0.44235 \mathrm{E}-04 \quad 0$ $0.43472 \mathrm{E}-04 \quad 0$ $0.42722 \mathrm{E}-04 \quad 0$. $0.41984 \mathrm{E}-04 \quad 0$. $0.41260 \mathrm{E}-04 \quad 0$. $0.4 C 548 E-04 \quad 0$ $0.39848 \mathrm{E}-04 \quad 0$ 0.39160 E- $04 \quad 0$. $0.38484 E-04 \quad 0$. $0.37820 \mathrm{E}-04 \quad 0$. $0.37168 E-04 \quad 0$. $0.36526 \mathrm{E}-04 \quad 0$. $0.35896 \mathrm{E}-04 \quad 0$. $0.35276 E-04 \quad 0$. $0.34667 \mathrm{E}-04 \quad 0$. $0.25628 E-04 \quad 0$. $0.25186 E-04 \quad 0$. $0.24751 \mathrm{E}-040$. $0.24324 \mathrm{E}-040$. $0.23904 \mathrm{E}-04 \quad 0$. $0.23491 \mathrm{E}-04 \quad 0$. $0.23086 E-04 \quad 0$. $0.22688 E-04 \quad 0$. $0.22296 \mathrm{E}-040$. $0.21911 E-04 \quad 0$. $0.21533 E-040$. $0.21161 E-040$. 0.20796 E-04 0. 0.20437 E-04 0 . $0.20085 E-04 \quad 0$. $0.19738 E-040$. $0.19397 E-04 \quad 0$. $0.19063 E-04 \quad 0$. $0.18734 \mathrm{E}-040$. $0.18410 E-04 \quad 0$. $0.18093 \mathrm{E}-04 \quad 0$. $0.17780 E-04 \quad 0$. $0.17473 t-04 \quad 0$. $0.17172 \mathrm{E}-04 \quad 0$. $0.16876 E-04 \quad 0$. 


$\begin{array}{llll}566 & 0.1936667 & 0.755530 \mathrm{E}-03 & 0 . \\ 567 & 0.1903243 & 0.726314 \mathrm{E}-03 & 0 . \\ 568 & 0.1870396 & 0.698458 \mathrm{E}-03 & 0 . \\ 569 & 0.1838116 & 0.671933 \mathrm{E}-03 & 0 . \\ 570 & 0.1806393 & 0.646656 \mathrm{E}-03 & 0 . \\ 571 & 0.1775217 & 0.622545 \mathrm{E}-03 & 0 . \\ 572 & 0.1744580 & 0.599532 \mathrm{E}-03 & 0 . \\ 573 & 0.1714471 & 0.577556 \mathrm{E}-03 & 0 . \\ 574 & 0.1684882 & 0.556561 \mathrm{E}-03 & 0 . \\ 575 & 0.1655804 & 0.536496 \mathrm{E}-03 & 0 . \\ 576 & 0.1627227 & 0.517314 \mathrm{E}-03 & 0 . \\ 577 & 0.1599144 & 0.498973 \mathrm{E}-03 & 0 . \\ 578 & 0.1571545 & 0.481434 \mathrm{E}-03 & 0 . \\ 579 & 0.1544423 & 0.464661 \mathrm{E}-03 & 0 . \\ 580 & 0.1517768 & 0.448616 \mathrm{E}-03 & 0 . \\ 581 & 0.1491574 & 0.433266 \mathrm{E}-03 & 0 . \\ 582 & 0.1465832 & 0.418578 \mathrm{E}-03 & 0 . \\ 583 & 0.1440534 & 0.404520 \mathrm{E}-03 & 0 . \\ 584 & 0.1415672 & 0.391061 \mathrm{E}-03 & 0 . \\ 585 & 0.1391240 & 0.378171 \mathrm{E}-03 & 0 . \\ 586 & 0.1367230 & 0.365824 \mathrm{E}-03 & 0 . \\ 587 & 0.1343633 & 0.353991 \mathrm{E}-03 & 0 . \\ 588 & 0.1320444 & 0.342650 \mathrm{E}-03 & 0 . \\ 589 & 0.1297655 & 0.331775 \mathrm{E}-03 & 0 . \\ 590 & 0.1275260 & 0.321346 \mathrm{E}-03 & 0 . \\ 591 & 0.1253251 & 0.311342 \mathrm{E}-03 & 0 . \\ 592 & 0.1231622 & 0.301741 \mathrm{E}-03 & 0 . \\ 593 & 0.1210366 & 0.292526 \mathrm{E}-03 & 0 . \\ 594 & 0.1189477 & 0.283678 \mathrm{E}-03 & 0 . \\ 595 & 0.1168948 & 0.275181 \mathrm{E}-03 & 0 . \\ 596 & 0.1148774 & 0.267018 \mathrm{E}-03 & 0 . \\ 597 & 0.1128948 & 0.259172 \mathrm{E}-03 & 0 . \\ 598 & 0.1109464 & 0.251631 \mathrm{E}-03 & 0 . \\ 599 & 0.1090317 & 0.244379 \mathrm{E}-03 & 0 . \\ 600 & 0.1071500 & 0.237404 \mathrm{E}-03 & 0 . \\ 601 & 0.1053007 & 0.230692 \mathrm{E}-03 & 0 . \\ 602 & 0.1034834 & 0.224232 \mathrm{E}-03 & 0 . \\ 603 & 0.1016974 & 0.218012 \mathrm{E}-03 & 0 . \\ 604 & 0.0999423 & 0.212022 \mathrm{E}-03 & 0 . \\ & 0.25 & & \\ 503 & & & \end{array}$

\begin{tabular}{|c|c|c|c|c|}
\hline $\begin{array}{l}0.208996 \mathrm{E} \\
0.208996 \mathrm{E} \\
0.208996 \mathrm{E} \\
0.208996 \mathrm{E} \\
0.208996 \mathrm{E} \\
0.208996 \mathrm{E} \\
0.208996 \mathrm{E} \\
0.208996 \mathrm{E} \\
0.208996 \mathrm{E} \\
0.208996 \mathrm{E} \\
0.208996 \mathrm{E} \\
0.208996 \mathrm{E} \\
0.208996 \mathrm{E} \\
0.208996 \mathrm{E} \\
0.208996 \mathrm{E} \\
0.208996 \mathrm{E} \\
0.208996 \mathrm{E} \\
0.208996 \mathrm{E} \\
0.208996 \mathrm{E} \\
0.208996 \mathrm{E} \\
0.208996 \mathrm{E} \\
0.208996 \mathrm{E} \\
0.208996 \mathrm{E} \\
0.208996 \mathrm{E} \\
0.208996 \mathrm{E} \\
0.208996 \mathrm{E} \\
0.208996 \mathrm{E} \\
0.208996 \mathrm{E} \\
0.208996 \mathrm{E} \\
0.208996 \mathrm{E} \\
0.208996 \mathrm{E} \\
0.208996 \mathrm{E} \\
0.208996 \mathrm{E} \\
0.208996 \mathrm{E} \\
0.208996 \mathrm{E} \\
0.208996 \mathrm{E} \\
0.208996 \mathrm{E} \\
0.208996 \mathrm{E} \\
0.208996 \mathrm{E}\end{array}$ & $\begin{array}{l}01 \\
01 \\
01 \\
01 \\
01 \\
01 \\
01 \\
01 \\
01 \\
01 \\
01 \\
01 \\
01 \\
01 \\
01 \\
01 \\
01 \\
01 \\
01 \\
01 \\
01 \\
01 \\
01 \\
01 \\
01 \\
01 \\
01 \\
01 \\
01 \\
01 \\
01 \\
01 \\
01 \\
01 \\
01 \\
01 \\
01 \\
01 \\
01\end{array}$ & $\begin{array}{l}0.103889 E-02 \\
0.997834 E-03 \\
0.958698 E-03 \\
0.92138 C E-03 \\
0.885787 E-03 \\
0.851830 E-03 \\
0.819428 E-03 \\
0.788504 E-03 \\
0.758984 E-03 \\
0.730799 E-03 \\
0.703885 E-03 \\
0.678178 E-03 \\
0.653620 E-03 \\
0.630154 E-03 \\
0.607726 E-03 \\
0.586287 E-03 \\
0.565787 E-03 \\
0.54618 C E-03 \\
0.527423 E-03 \\
0.509475 E-03 \\
0.492296 E-03 \\
0.475850 E-03 \\
0.460100 E-03 \\
0.445015 E-03 \\
0.430560 E-03 \\
0.416708 E-03 \\
0.403428 E-03 \\
0.390694 E-03 \\
0.378479 E-03 \\
0.366760 E-03 \\
0.355513 E-03 \\
0.344715 E-03 \\
0.334346 E-03 \\
0.324385 E-03 \\
0.314815 E-03 \\
0.305616 E-03 \\
0.296771 E-03 \\
0.288265 E-03 \\
0.280081 E-03\end{array}$ & $\begin{array}{l}0.25608 E-03 \\
0.24532 E-03 \\
0.23605 E-03 \\
0.22675 E-03 \\
0.21790 E-03 \\
0.20947 E-03 \\
0.20143 E-03 \\
0.19375 E-03 \\
0.18643 E-03 \\
0.17944 E-03 \\
0.17276 E-03 \\
0.16638 E-03 \\
0.16029 E-03 \\
0.15447 E-03 \\
0.14890 E-03 \\
0.14359 E-03 \\
0.13851 E-03 \\
0.13365 E-03 \\
0.12901 E-03 \\
0.12457 E-03 \\
0.12031 E-03 \\
0.11625 E-03 \\
0.11235 E-03 \\
0.10862 E-03 \\
0.10505 E-03 \\
0.10163 E-03 \\
0.98349 E-04 \\
0.95205 E-04 \\
0.92191 E-04 \\
0.89300 E-04 \\
0.86527 E-04 \\
0.83865 E-04 \\
0.81311 E-04 \\
0.78858 E-04 \\
0.76501 E-04 \\
0.74237 E-04 \\
0.72062 E-04 \\
0.69970 E-04 \\
0.67958 E-04\end{array}$ & $\begin{array}{l}0.16584 \mathrm{E}-04 \\
0.16298 \mathrm{E}-04 \\
0.16017 \mathrm{E}-04 \\
0.15740 \mathrm{E}-04 \\
0.15469 \mathrm{E}-04 \\
0.15202 \mathrm{E}-04 \\
0.14939 \mathrm{E}-04 \\
0.14682 \mathrm{E}-04 \\
0.14428 \mathrm{E}-04 \\
0.14179 \mathrm{E}-04 \\
0.13934 \mathrm{E}-04 \\
0.13694 \mathrm{E}-04 \\
0.13458 \mathrm{E}-04 \\
0.13225 \mathrm{E}-04 \\
0.12997 \mathrm{E}-04 \\
0.12773 \mathrm{E}-04 \\
0.12552 \mathrm{E}-04 \\
0.12336 \mathrm{E}-04 \\
0.12123 \mathrm{E}-04 \\
0.11914 \mathrm{E}-04 \\
0.11708 \mathrm{E}-04 \\
0.11506 \mathrm{E}-04 \\
0.11307 \mathrm{E}-04 \\
0.11112 \mathrm{E}-04 \\
0.10920 \mathrm{E}-04 \\
0.10732 \mathrm{E}-04 \\
0.11547 \mathrm{E}-04 \\
0.10365 \mathrm{E}-04 \\
0.10186 \mathrm{E}-04 \\
0.10010 \mathrm{E}-04 \\
0.98373 \mathrm{E}-05 \\
0.96675 \mathrm{E}-05 \\
0.95007 \mathrm{E}-05 \\
0.93367 \mathrm{E}-05 \\
0.91756 \mathrm{E}-05 \\
0.90172 \mathrm{E}-05 \\
0.88616 \mathrm{E}-05 \\
0.87087 \mathrm{E}-05 \\
0.85584 \mathrm{E}-05\end{array}$ \\
\hline
\end{tabular}


hCBO Equivalent

BUCKLING $=0.002362$

\begin{tabular}{|c|c|c|c|c|c|c|c|c|c|c|c|}
\hline $\begin{array}{r}G P \\
1\end{array}$ & $\begin{array}{c}\text { FLUX } \\
1.475992\end{array}$ & $\begin{array}{l}\text { FISSIDN } \\
\text { SPECTRUM } \\
0.132000\end{array}$ & $\begin{array}{l}\text { NU SIGMA } \\
\text { FISSION } \\
0.037210\end{array}$ & $\begin{array}{l}\text { AB SORP TION } \\
0.083414\end{array}$ & $\begin{array}{c}3 \text { SIGMA } \\
\text { TRANSPORT } \\
0.392549\end{array}$ & $\begin{array}{c}\mathrm{J} T C_{\mathrm{J}} \mathrm{J+1} \\
0.019192\end{array}$ & 0.012683 & 0.014027 & $\begin{array}{c}\text { ASTICS } \\
4 \\
0.011521\end{array}$ & 0.006949 & $\begin{array}{c}6 \\
0.005720\end{array}$ \\
\hline 2 & 3.003948 & 0.213000 & 0.033407 & 0.074865 & 0.431765 & 0.022993 & 0.010711 & 0.011885 & 0.008947 & 0.004764 & 0.003852 \\
\hline 3 & 4.344786 & 0.232000 & 0.025658 & 0.068959 & 0.508622 & 0.036065 & 0.016850 & 0.003768 & 0.000635 & 0.001376 & 0.000189 \\
\hline 4 & 5.652061 & 0.179000 & 0.009390 & 0.065393 & 0.704041 & 0.051443 & 0.005227 & 0.002350 & 0.000790 & 0.000395 & -0 \\
\hline 5 & 12.419576 & 0.116000 & 0.007876 & 0.039214 & 0.642669 & 0.033858 & 0.000263 & 0.000136 & 0.000040 & 0.000036 & -0 \\
\hline 6 & 8.972924 & 0.067000 & 0.007797 & 0.061171 & 0.978570 & 0.056274 & 0.000051 & 0.000019 & 0.000011 & 0.000006 & 0.000005 \\
\hline 7 & 11.555861 & 0.034000 & 0.007653 & 0.047767 & 0.940792 & $0.0425 \mathrm{C6}$ & 0.000008 & 0.000000 & 0.000000 & 0.000000 & 0.000002 \\
\hline 8 & 11.212336 & 0.017000 & 0.007854 & 0.045150 & 1.014016 & 0.036079 & 0.002816 & 0.000002 & 0.000000 & 0.000000 & -0 \\
\hline 9 & 8.633724 & 0.010000 & 0.008171 & 0.046333 & 1.114180 & 0.036810 & 0.000899 & 0.000618 & 0.000000 & -0 & -0 \\
\hline 10 & 7.357265 & 0. & 0.009048 & 0.045533 & 1.163943 & 0.034032 & 0.001067 & 0.000407 & -0 & -0 & -0 \\
\hline 11 & 5.158281 & 0 & 0.009048 & 0.048336 & 1.371255 & 0.038213 & 0.000000 & -0 & -0 & -0 & -0 \\
\hline 12 & 4.313967 & 0. & 0.010179 & 0.046862 & 1.246432 & 0.032912 & -0 & -0 & -0 & -0 & -0 \\
\hline 13 & 2.870952 & 0. & 0.010179 & 0.048847 & 1.425599 & 0.034816 & -0 & -0 & -0 & -0 & -0 \\
\hline 14 & 2.430732 & 0. & 0.011672 & 0.039790 & 1.773461 & 0.026855 & -0 & $-c$ & -0 & -0 & -0 \\
\hline 15 & 1.770665 & 0. & 0.017689 & 0.035477 & 1.701016 & 0.011717 & -0 & -0 & -0 & -0 & -0 \\
\hline 16 & 0.424068 & 0. & 0.035332 & 0.047611 & 1.798076 & 0.010014 & -0 & -0 & -0 & -0 & -0 \\
\hline 17 & 0.050229 & 0. & 0.079622 & 0.083409 & 2.086336 & 0.007788 & -0 & -0 & -0 & -0 & -0 \\
\hline 18 & 0.003919 & 0. & 0.076456 & 0.099250 & 4.065413 & 0. & 0. & 0. & 0. & 0. & 0. \\
\hline
\end{tabular}

